Simon Stephan, Monika Thol, Jadran Vrabec, Hans Hasse

\title{
Thermophysical Properties of the Lennard- Jones Fluid: Database and Data Assessment
}

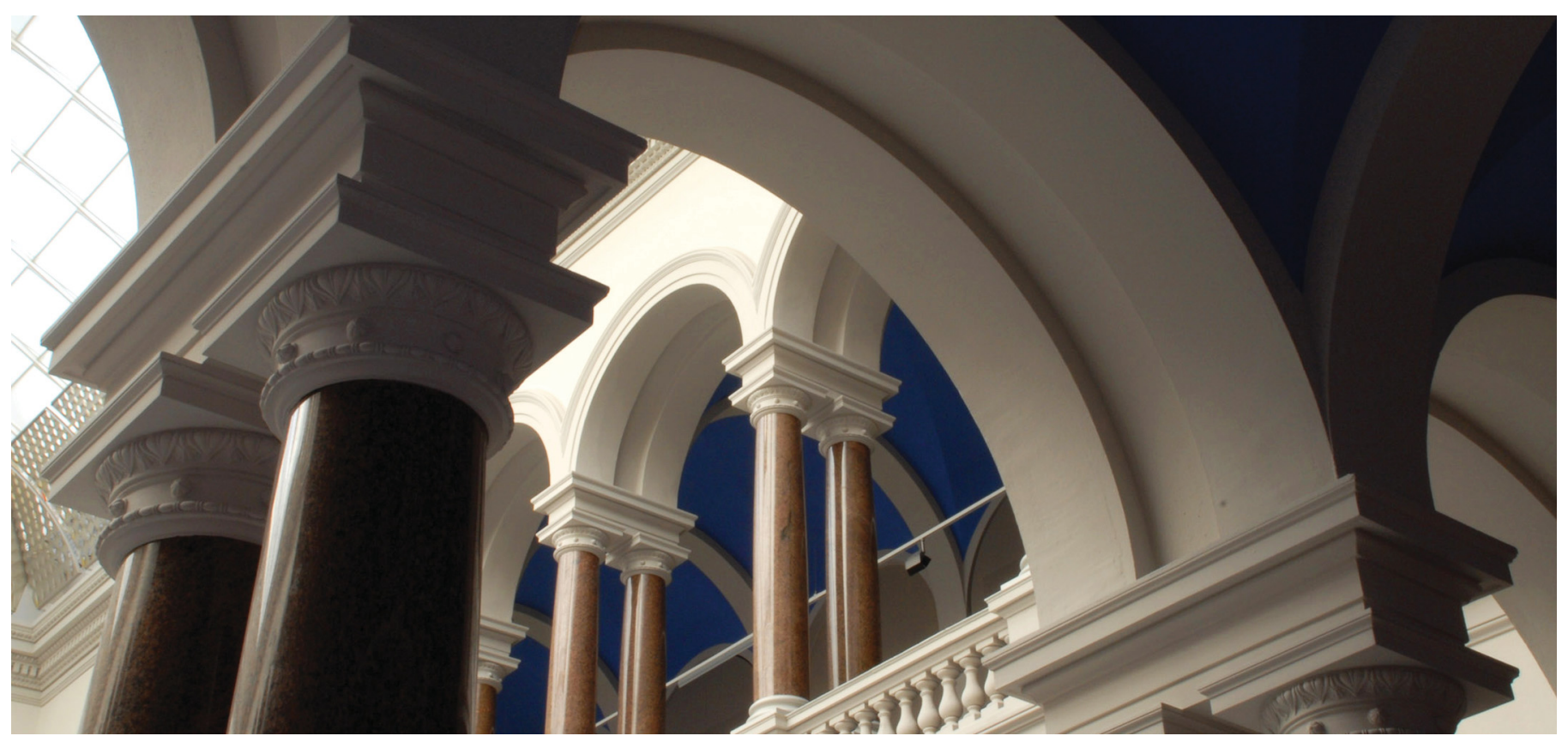

Stephan, S., Thol, M., Vrabec, J., \& Hasse, H. (2019). Thermophysical Properties of the Lennard-Jones Fluid: Database and Data Assessment. Journal of Chemical Information and Modeling, 59(10), 42484265. https://doi.org/10.1021/acs.jcim.9b00620 


\title{
Thermophysical Properties of the Lennard-Jones Fluid: Database and Data Assessment
}

\author{
Simon Stephan, ${ }^{*, \dagger}$ Monika Thol, ${ }^{\ddagger}$ Jadran Vrabec, $₫$ and Hans Hasse ${ }^{\dagger}$ \\ $\dagger$ Laboratory of Engineering Thermodynamics (LTD), TU Kaiserslautern, Kaiserslautern \\ 67663, Germany \\ $\ddagger$ Thermodynamics, Ruhr University Bochum, Bochum 44801, Germany \\ IThermodynamics and Process Engineering, TU Berlin, Berlin 10587, Germany \\ E-mail: *simon.stephan@mv.uni-kl.de
}

\begin{abstract}
Literature data on thermophysical properties of the Lennard-Jones fluid, which were sampled with Molecular Dynamics and Monte Carlo simulations, were reviewed and assessed. The literature data was complemented by simulation data from the present work that was taken in regions in which previously only sparse data was available. Data on homogeneous state points (for given temperature $T$ and density $\rho$ : pressure $p$, thermal expansion coefficient $\alpha$, isothermal compressibility $\beta$, thermal pressure coefficient $\gamma$, internal energy $u$, isochoric heat capacity $c_{v}$, isobaric heat capacity $c_{p}$, Grüneisen coefficient $\Gamma$, Joule-Thomson coefficient $\mu_{\mathrm{JT}}$, speed of sound $w$, Helmholtz energy $a$, chemical potential $\mu$, surface tension $\gamma$ ) was considered as well as data on the vapor-liquid equilibrium (for given $T$ : vapor pressure $p^{s}$, saturated liquid and vapor densities $\rho^{\prime}$ and $\rho^{\prime \prime}$, enthalpy of vaporization $\Delta h_{\mathrm{v}}$ ). The entire set of available data, which contains about 35,000 data points, was digitalized and included in a database, which is made available in the electronic supplementary material of this paper. Different consistency tests were applied to assess the accuracy and precision of the data. The data on homogeneous states were evaluated point-wise using data from their respective
\end{abstract}


vicinity and equations of state. Approximately 5\% of all homogeneous bulk data were discarded as outliers. The vapor-liquid equilibrium data were assessed by tests based on the compressibility factor, the Clausius-Clapeyron equation, and by an outlier test. Seven particularly reliable vapor-liquid equilibrium data sets were identified. The mutual agreement of these data sets is approximately $\pm 1 \%$ for the vapor pressure, $\pm 0.2 \%$ for the saturated liquid density, $\pm 1 \%$ for the saturated vapor density, and $\pm 0.75 \%$ for the enthalpy of vaporization excluding the region close to the critical point.

\section{Introduction}

The assessment of experimental thermophysical property data is a well established field in chemical engineering, ${ }^{1-3}$ especially for phase equilibrium data. ${ }^{4,5}$ For instance, consistency tests based on thermodynamic limits or balance equations are used to evaluate the quality of data sets or data points of a given thermophysical property. However, such approaches are not common in the analysis of thermophysical property data obtained by computer experiments, like molecular dynamics (MD) or Monte Carlo (MC) simulations. Also, thermophysical data repositories ${ }^{6,7}$ have so far not addressed molecular simulation data of model fluids, like the Lennard-Jones fluid. The present work therefore provides a coherent and consolidated database of thermophysical property data of the Lennard-Jones fluid.

The Lennard-Jones potential ${ }^{8,9}$ is of fundamental importance for the development of theories and methods in soft matter physics, ${ }^{10-12}$ and has been widely used since the early days of computer simulation. ${ }^{13-20}$ It is often taken as a benchmark for the validation of simulation codes and the test of new simulation techniques. Despite its simplicity, the Lennard-Jones model fluid yields a realistic representation of simple fluids. ${ }^{21,22}$ Due to its importance, it is sometimes even referred to as Lennard-Jonesium ${ }^{23-25}$ - suggesting that it is viewed as a chemical element. ${ }^{26}$ The $^{2}$ Lennard-Jones potential is defined as the pairwise additive and spherically symmetric potential

$$
u_{\mathrm{LJ}}(r)=4 \varepsilon\left[\left(\frac{\sigma}{r}\right)^{12}-\left(\frac{\sigma}{r}\right)^{6}\right]
$$


where $r$ is the distance between two particles. Its parameters $\varepsilon$ and $\sigma$ characterize the size of the particles and the magnitude of their dispersive attraction, respectively. Simulations are usually performed with a truncated potential in combination with a long-range correction. ${ }^{27}$

To sample thermophysical properties of the Lennard-Jones fluid, computer experiments are carried out. In general, an obtained simulation result of a given observable $x^{\text {sim }}$ will not agree with the true model value $x^{\bmod } .{ }^{28}$ Like in experiments in the laboratory, also in computer experiments errors occur ${ }^{29-33}$ that can cause deviations between the exact true value $x^{\text {mod }}$ and the value observed in the simulation $x^{\text {sim }}$. Both stochastic and systematic errors are usually present to some extent in computer experiments. Techniques to assess statistical errors are well established for computer simulations. ${ }^{27,30,34}$ It is more difficult to assess systematic simulation errors. As in laboratory experiments, round robin studies can be used for doing this, in which the same simulation task is carried out by different groups with different programs. It is known that the results from such studies generally differ by more that the combined statistical uncertainty of the individual data. E.g., the results obtained by repetition usually differ due to varying methods or simulation programs etc. ${ }^{29}$ Systematic errors may be a consequence of erroneous algorithms, ${ }^{35}$ user errors, differences due to different simulation methods (for example MD and MC, phase equilibrium simulation methods, techniques for the determination of the chemical potential ${ }^{36}$ etc.). Systematic errors may furthermore be caused by finite size effects, erroneous evaluation of long range interactions, insufficient equilibration or production periods, compilers, parallelization, and hardware architecture. ${ }^{29}$ Last but not least, also typographical errors in publications have to be considered as a possible error source. The detection and assessment of outliers in large data sets is a standard task in the field of data science ${ }^{37-39}$ and is widely applied to experimental data, ${ }^{40,41}$ but has to the best of our knowledge not yet been applied to thermophysical property data obtained by computer experiments with model fluids. In the present work, we use the terms accurate \& precise as follows: accurate simulation results $x^{\mathrm{sim}}$ scatter around the true value $x^{\mathrm{mod}}$ without trend; precise means here that given simulation results $x^{\text {sim }}$ are both accurate and exhibit a low scattering.

This article reviews and assesses molecular simulation data of the Lennard-Jones fluid. Approximately 35,000 data points were taken into account, including new simulation data from this 
work that were taken to complement the available data in regions that were only sparsely investigated in the literature. Vapor-liquid equilibrium (VLE) data and data on state points from the homogeneous regions were considered: For the VLE, the vapor pressure, the saturated densities, and the enthalpy of vaporization were investigated; for homogeneous state points, the investigated properties are: the pressure, thermal expansion coefficient, isothermal compressibility, thermal pressure coefficient, internal energy, isochoric heat capacity, isobaric heat capacity, Grüneisen coefficient, Joule-Thomson coefficient, speed of sound, Helmholtz energy, chemical potential, and surface tension. Transport properties were not taken into account.

The data were assessed by consistency tests to provide an indication for their accuracy and precision. The entire database was digitalized and is presented in a consistent form as a spreadsheet in the supplementary material. The database also contains flags that indicate whether a data point was identified as an outlier. All thermophysical property data are reported using reduced units with respect to the Lennard-Jones energy and size parameters $\varepsilon$ and $\sigma$ as well as the Boltzmann constant $k_{\mathrm{B}} \cdot{ }^{27}$

\section{Molecular simulation data of the Lennard-Jones fluid}

Table 1 gives an overview on the thermophysical property data of the Lennard-Jones fluid from the literature. Only data on homogeneous states and vapor-liquid equilibria were considered in this work. Data on the second and third virial coefficients and the so-called characteristic curves ${ }^{42,43}$ were included in the database, but not further assessed regarding their accuracy. The vast majority of studies in the literature report $p v T$ and internal energy data for homogeneous state points, cf. Table 1. The chemical potential and higher order derivatives, like speed of sound, heat capacities, or isothermal compressibility, were less frequently investigated. Wherever statistical uncertainties were reported in the literature, they were also included in the database. 
Table 1: Computer experiment data of the Lennard-Jones fluid from the literature. The data are sorted chronologically for each property. The \# is the number of data points and $T$ the investigated temperature range.

\begin{tabular}{|c|c|c|c|}
\hline Authors & Year & $\#$ & $T$ \\
\hline \multicolumn{4}{|l|}{$p v T$ data } \\
\hline Wood and Parker ${ }^{15}$ & 1957 & 13 & 2.74 \\
\hline Fickett and Wood ${ }^{44}$ & 1960 & 23 & $1.92-177.95$ \\
\hline McDonald and Singer ${ }^{45}$ & 1967 & 28 & $0.72-1.24$ \\
\hline McDonald and Singer 19 & 1967 & 48 & $1.45-3.53$ \\
\hline Verlet and Levesque ${ }^{46}$ & 1967 & 7 & $1.05-2.74$ \\
\hline Verlet $^{18}$ & 1967 & 39 & $0.59-4.63$ \\
\hline Wood $^{47}$ & 1968 & 41 & $1.06-100$ \\
\hline Hansen and Verlet ${ }^{20}$ & 1969 & 25 & $0.75-1.15$ \\
\hline Levesque and Verlet ${ }^{48}$ & 1969 & 25 & $0.72-3.67$ \\
\hline McDonald and Singer 49 & 1969 & 28 & $0.72-1.24$ \\
\hline Hansen $^{50}$ & 1970 & 9 & $2.74-100$ \\
\hline McDonald and Woodcock ${ }^{51}$ & 1970 & 6 & $0.75-2.33$ \\
\hline Toxvaerd and Praestgaard ${ }^{52}$ & 1970 & 8 & 1.35 \\
\hline Weeks et al. ${ }^{53}$ & 1971 & 5 & $0.75-1.35$ \\
\hline McDonald and Singer 54 & 1972 & 56 & $0.55-1.24$ \\
\hline Schofield ${ }^{55}$ & 1973 & 6 & $0.73-1.1$ \\
\hline Street et al. ${ }^{56}$ & 1974 & 80 & $0.75-3.05$ \\
\hline $\operatorname{Adams}^{57}$ & 1975 & 12 & $2-4$ \\
\hline$A d a m s^{58}$ & 1976 & 16 & $1-1.2$ \\
\hline Carley ${ }^{59}$ & 1977 & 11 & 1.35 \\
\hline Adams $^{60}$ & 1979 & 31 & $1.15-1.35$ \\
\hline Nicolas et al. ${ }^{61}$ & 1979 & 108 & $0.48-6.01$ \\
\hline$R e e^{62}$ & 1980 & 11 & $0.81-2.7$ \\
\hline Yao et al. ${ }^{63}$ & 1982 & 12 & $1.15-1.25$ \\
\hline Powles et al. ${ }^{64}$ & 1982 & 37 & $0.7-1.41$ \\
\hline Lucas $^{65}$ & 1986 & 10 & $0.79-1.83$ \\
\hline Shaw $^{66}$ & 1988 & 265 & $0.6-136.25$ \\
\hline Adachi et al. ${ }^{67}$ & 1988 & 328 & $0.7-2.95$ \\
\hline Baranyai et al. ${ }^{68}$ & 1989 & 12 & $0.75-1.5$ \\
\hline Saager and Fischer 69 & 1990 & 38 & $0.57-4$ \\
\hline Sowers and Sandler 70 & 1991 & 60 & $1.35-6$ \\
\hline Lotfi et al. ${ }^{71}$ & 1992 & 19 & $0.7-1.3$ \\
\hline Giaquinta et al. ${ }^{72}$ & 1992 & 12 & $0.75-1.15$ \\
\hline Johnson et al. ${ }^{73}$ & 1993 & 199 & $0.7-6$ \\
\hline Kolafa et al. ${ }^{74}$ & 1993 & 43 & $0.72-4.85$ \\
\hline Miyano $^{75}$ & 1993 & 112 & $0.45-100$ \\
\hline Kolafa and Nezbeda ${ }^{76}$ & 1994 & 13 & $0.81-10$ \\
\hline Lustig $^{77}$ & 1994 & 2 & 1.18 \\
\hline
\end{tabular}

Continued on next page 


\begin{tabular}{|c|c|c|c|}
\hline Authors & Year & $\#$ & $T$ \\
\hline Mecke et al. ${ }^{78}$ & 1996 & 12 & $1.32-1.34$ \\
\hline Roccatano et al. ${ }^{79}$ & 1998 & 10 & $1.4-10$ \\
\hline Meier 80 & 2002 & 351 & $0.7-6$ \\
\hline Linhart et al. ${ }^{81}$ & 2005 & 108 & $0.7-1.2$ \\
\hline Morsali et al. ${ }^{82}$ & 2007 & 13 & 5.01 \\
\hline Baidakov et al. ${ }^{83}$ & 2008 & 208 & $0.35-2$ \\
\hline Lustig $^{84}$ & 2011 & 8 & $1.02-3$ \\
\hline May and Mausbach ${ }^{85}$ & 2012 & 205 & $0.69-6.17$ \\
\hline Yigzawe and Sadus ${ }^{86,87}$ & 2012 & 406 & $1.3-2.62$ \\
\hline Mairhofer and Sadus ${ }^{88,89}$ & 2013 & 282 & $1.36-3.05$ \\
\hline Thol et al. ${ }^{90}$ & 2016 & 197 & $0.7-9$ \\
\hline Deiters and Neumaier ${ }^{43}$ & 2016 & 255 & $0.67-22.54$ \\
\hline Köster et al. ${ }^{91}$ & 2017 & 45 & $1.01-30$ \\
\hline Köster et al. ${ }^{91,92}$ & 2017 & 65 & $1.01-30$ \\
\hline Ustinov $^{93,94}$ & 2017 & 232 & $0.76-1.14$ \\
\hline Schultz and Kofke $e^{95}$ & 2018 & 404 & $0.68-2272$ \\
\hline this work & 2019 & 655 & $0.7-90$ \\
\hline \multicolumn{4}{|l|}{ Internal energy $u$} \\
\hline Wood and Parker ${ }^{15}$ & 1957 & 13 & 2.74 \\
\hline McDonald and Singer ${ }^{45}$ & 1967 & 27 & $0.72-1.24$ \\
\hline McDonald and Singer ${ }^{19}$ & 1967 & 48 & $1.45-3.53$ \\
\hline Verlet $^{18}$ & 1967 & 39 & $0.59-4.63$ \\
\hline Verlet and Levesque ${ }^{46}$ & 1967 & 7 & $1.05-2.74$ \\
\hline Wood $^{47}$ & 1968 & 41 & $1.06-100$ \\
\hline Levesque and Verlet ${ }^{48}$ & 1969 & 25 & $0.72-3.67$ \\
\hline McDonald and Singer ${ }^{49}$ & 1969 & 28 & $0.72-1.24$ \\
\hline Hansen $^{50}$ & 1970 & 9 & $2.74-100$ \\
\hline McDonald and Woodcock 51 & 1970 & 6 & $0.75-2.33$ \\
\hline Weeks et al. ${ }^{53}$ & 1971 & 5 & $0.75-1.35$ \\
\hline McDonald and Singer ${ }^{54}$ & 1972 & 56 & $0.55-1.24$ \\
\hline Street et al. ${ }^{56}$ & 1974 & 80 & $0.75-3.05$ \\
\hline $\operatorname{Adams}^{57}$ & 1975 & 12 & $2-4$ \\
\hline Adams $^{58}$ & 1976 & 16 & $1-1.2$ \\
\hline Torrie and Valleau ${ }^{96}$ & 1977 & 7 & $0.092-1.35$ \\
\hline Adams $^{60}$ & 1979 & 31 & $1.15-1.35$ \\
\hline Nicolas et al. ${ }^{61}$ & 1979 & 108 & $0.48-6.01$ \\
\hline$R e e^{62}$ & 1980 & 11 & $0.81-2.7$ \\
\hline Yao et al. ${ }^{63}$ & 1982 & 12 & $1.15-1.25$ \\
\hline Lucas $^{65}$ & 1986 & 10 & $0.79-1.83$ \\
\hline$S_{h a w}^{66}$ & 1988 & 265 & $0.59-136.25$ \\
\hline Baranyai et al. ${ }^{68}$ & 1989 & 18 & $0.75-1.5$ \\
\hline Saager and Fischer ${ }^{69}$ & 1990 & 38 & $0.57-4$ \\
\hline Sowers and Sandler ${ }^{70}$ & 1991 & 54 & $1.35-6$ \\
\hline
\end{tabular}

Continued on next page 


\begin{tabular}{|c|c|c|c|}
\hline Authors & Year & $\#$ & $T$ \\
\hline Lotfi et al. ${ }^{71}$ & 1992 & 19 & $0.7-1.3$ \\
\hline Giaquinta et al. ${ }^{72}$ & 1992 & 12 & $0.75-1.15$ \\
\hline Johnson et al. ${ }^{73}$ & 1993 & 199 & $0.7-6$ \\
\hline Kolafa et al..$^{74}$ & 1993 & 43 & $0.72-4.85$ \\
\hline Miyano $^{75}$ & 1993 & 112 & $0.45-100$ \\
\hline Kolafa and Nezbeda ${ }^{76}$ & 1994 & 13 & $0.81-10$ \\
\hline Lustig $^{77}$ & 1994 & 2 & 1.18 \\
\hline Mecke et al. ${ }^{78}$ & 1996 & 12 & $1.32-1.34$ \\
\hline Roccatano et al. ${ }^{79}$ & 1998 & 10 & $1.4-10$ \\
\hline Meier 80 & 2002 & 351 & $0.7-6$ \\
\hline Baidakov et al. ${ }^{83}$ & 2008 & 201 & $0.35-2$ \\
\hline May and Mausbach ${ }^{85}$ & 2012 & 218 & $0.68-6.17$ \\
\hline Yigzawe and Sadus ${ }^{86,87}$ & 2012 & 346 & $1.31-2.62$ \\
\hline Mairhofer and Sadus ${ }^{88,89}$ & 2013 & 282 & $1.36-3.05$ \\
\hline Thol et al. ${ }^{90}$ & 2016 & 197 & $0.7-9$ \\
\hline Deiters and Neumaier ${ }^{43,97}$ & 2016 & 255 & $0.67-22.54$ \\
\hline Köster et al. ${ }^{91}$ & 2017 & 45 & $1.01-30$ \\
\hline Köster et al. ${ }^{91,92}$ & 2017 & 65 & $1.01-30$ \\
\hline Ustinov $^{93,94}$ & 2017 & 232 & $0.76-1.14$ \\
\hline Schultz and Kofke $e^{95}$ & 2018 & 404 & $0.68-2272$ \\
\hline this work & 2019 & 655 & $0.7-90$ \\
\hline \multicolumn{4}{|l|}{$\operatorname{VLE} p^{s}, \rho^{\prime}, \rho^{\prime \prime}, \Delta h_{v}$} \\
\hline Hansen and $\operatorname{Verlet}^{20}\left(p^{s}, \rho^{\prime}, \rho^{\prime \prime}, \Delta h_{v}\right)$ & 1969 & 8 & $0.75-1.15$ \\
\hline Lee et al. ${ }^{98}\left(\rho^{\prime}, \rho^{\prime \prime}\right)$ & 1974 & 10 & $0.7-1.2$ \\
\hline $\operatorname{Adams}^{58}\left(p^{s}, \rho^{\prime}, \rho^{\prime \prime}, \Delta h_{v}\right)$ & 1976 & 44 & $0.6-1.1$ \\
\hline Chapela et al. ${ }^{99}\left(\rho^{\prime}, \rho^{\prime \prime}\right)$ & 1977 & 6 & $0.7-0.84$ \\
\hline $\operatorname{Adams}^{60}\left(p^{s}, \rho^{\prime}, \rho^{\prime \prime}, \Delta h_{v}\right)$ & 1979 & 11 & $1.15-1.30$ \\
\hline Panagiotopoulos ${ }^{100}\left(p^{s}, \rho^{\prime}, \rho^{\prime \prime}, \Delta h_{v}\right)$ & 1987 & 30 & $0.75-1.3$ \\
\hline Panagiotopoulos et al. ${ }^{101}\left(p^{s}, \rho^{\prime}, \rho^{\prime \prime}, \Delta h_{v}\right)$ & 1988 & 30 & $0.75-1.3$ \\
\hline Nijmeijer et al. ${ }^{102}\left(\rho^{\prime}, \rho^{\prime \prime}\right)$ & 1988 & 2 & 0.92 \\
\hline Smit and Frenkel ${ }^{103}\left(p^{s}, \rho^{\prime}, \rho^{\prime \prime}, \Delta h_{v}\right)$ & 1989 & 20 & $1.15-1.3$ \\
\hline Lotfi et al. ${ }^{71}\left(p^{s}, \rho^{\prime}, \rho^{\prime \prime}\right)$ & 1992 & 52 & $0.7-1.3$ \\
\hline$K_{o f k e}{ }^{104}\left(p^{s}, \rho^{\prime}, \rho^{\prime \prime}, \Delta h_{v}\right)$ & 1993 & 80 & $0.74-1.32$ \\
\hline Holcomb et al. ${ }^{105}\left(\rho^{\prime}, \rho^{\prime \prime}\right)$ & 1993 & 6 & $0.72-1.13$ \\
\hline Agrawal and Kofke $e^{106}\left(p^{s}, \rho^{\prime}, \rho^{\prime \prime}, \Delta h_{v}\right)$ & 1995 & 52 & $0.68-0.74$ \\
\hline Hunter and Reinhardt ${ }^{107}\left(\rho^{\prime}, \rho^{\prime \prime}\right)$ & 1995 & 39 & $1-1.35$ \\
\hline Sadus and Prausnitz ${ }^{108}\left(p^{s}, \rho^{\prime}, \rho^{\prime \prime}, \Delta h_{v}\right)$ & 1996 & 30 & $1-1.25$ \\
\hline Mecke et al. ${ }^{109}\left(\rho^{\prime}, \rho^{\prime \prime}\right)$ & 1997 & 6 & $0.7-1.1$ \\
\hline Plačkov and Sadus ${ }^{110}\left(p^{s}, \rho^{\prime}, \rho^{\prime \prime}, \Delta h_{v}\right)$ & 1997 & 55 & $0.95-1.27$ \\
\hline Guo et al. ${ }^{111}\left(\rho^{\prime}, \rho^{\prime \prime}\right)$ & 1997 & 10 & $0.75-1.25$ \\
\hline Guo and $L u^{112}\left(\rho^{\prime}, \rho^{\prime \prime}\right)$ & 1997 & 8 & $0.75-1.15$ \\
\hline Martin and Siepmann ${ }^{113}\left(p^{s}, \rho^{\prime}, \rho^{\prime \prime}\right)$ & 1998 & 18 & $0.75-1.18$ \\
\hline Trokhymchuk and Alejandre ${ }^{114}\left(p^{s}, \rho^{\prime}, \rho^{\prime \prime}\right)$ & 1999 & 18 & $0.72-1.27$ \\
\hline
\end{tabular}




\begin{tabular}{|c|c|c|c|}
\hline Authors & Year & $\#$ & $T$ \\
\hline Anisimov et al. ${ }^{115}\left(p^{s}, \rho^{\prime}, \rho^{\prime \prime}\right)$ & 1999 & 18 & $0.75-1$ \\
\hline Potoff and Panagiotopoulos ${ }^{116}\left(\rho^{\prime}, \rho^{\prime \prime}\right)$ & 2000 & 36 & $0.95-1.31$ \\
\hline Baidakov et al. ${ }^{117}\left(p^{s}, \rho^{\prime}, \rho^{\prime \prime}\right)$ & 2000 & 21 & $0.72-1.23$ \\
\hline Okumura and Yonezawa ${ }^{118,119}\left(p^{s}, \rho^{\prime}, \rho^{\prime \prime}\right)$ & 2000 & 39 & $0.7-1.3$ \\
\hline Shi and Johnson ${ }^{120}\left(\rho^{\prime}, \rho^{\prime \prime}\right)$ & 2001 & 26 & $1.15-1.27$ \\
\hline Chen et al. ${ }^{24}\left(\rho^{\prime}, \rho^{\prime \prime}\right)$ & 2001 & 6 & $0.7-0.8$ \\
\hline Okumura and Yonezawa ${ }^{121,122}\left(p^{s}, \rho^{\prime}, \rho^{\prime \prime}\right)$ & 2001 & 30 & $1.25-1.32$ \\
\hline Baidakov et al. ${ }^{123}\left(\rho^{\prime}, \rho^{\prime \prime}\right.$, spinodal $)$ & 2002 & 14 & $0.72-1.23$ \\
\hline Kioupis et al. ${ }^{124}\left(p^{s}, \rho^{\prime}, \rho^{\prime \prime}, \Delta h_{v}\right)$ & 2002 & 40 & $1.03-1.3$ \\
\hline Errington ${ }^{125}\left(p^{s}, \rho^{\prime}, \rho^{\prime \prime}\right)$ & 2003 & 12 & $0.7-1.3$ \\
\hline Errington $^{126,127}\left(p^{s}, \rho^{\prime}, \rho^{\prime \prime}\right)$ & 2003 & 39 & $0.7-1.3$ \\
\hline Stoll et al. ${ }^{128}\left(p^{s}, \rho^{\prime}, \rho^{\prime \prime}, \Delta h_{v}\right)$ & 2003 & 44 & $0.73-1.26$ \\
\hline Baidakov et al. ${ }^{129}\left(p^{s}, \rho^{\prime}, \rho^{\prime \prime}\right)$ & 2007 & 36 & $0.5-1.2$ \\
\hline Betancourt-Cárdenas et al. ${ }^{130}\left(p^{s}, \rho^{\prime}, \rho^{\prime \prime}, \Delta h_{v}\right)$ & 2008 & 35 & $0.7-1.27$ \\
\hline$J_{\text {aneček }}^{131}\left(p^{s}, \rho^{\prime}, \rho^{\prime \prime}, \Delta h_{v}\right)$ & 2009 & 16 & $0.72-1.25$ \\
\hline Galliero et al. ${ }^{132}\left(\rho^{\prime}, \rho^{\prime \prime}\right)$ & 2009 & 38 & $0.7-1.3$ \\
\hline Sadus ${ }^{133}\left(p^{s}\right)$ & 2012 & 14 & $0.7-1.29$ \\
\hline Mick et al. ${ }^{23}\left(p^{s}, \rho^{\prime}, \rho^{\prime \prime}, \Delta h_{v}\right)$ & 2013 & 36 & $0.75-1.3$ \\
\hline Martinez-Ruiz et al. ${ }^{134}\left(p^{s}, \rho^{\prime}, \rho^{\prime \prime}\right)$ & 2014 & 21 & $0.7-1.1$ \\
\hline Janeček et al. ${ }^{135}\left(p^{s}, \rho^{\prime}, \rho^{\prime \prime}, \Delta h_{v}\right)$ & 2017 & 31 & $0.7-1.25$ \\
\hline Janeček et al. ${ }^{135,136}\left(p^{s}, \rho^{\prime}, \rho^{\prime \prime}, \Delta h_{v}\right)$ & 2017 & 24 & $0.8-1.25$ \\
\hline Werth et al. ${ }^{137}\left(p^{s}, \rho^{\prime}, \rho^{\prime \prime}, \Delta h_{v}\right)$ & 2017 & 36 & $0.72-1.24$ \\
\hline Stephan and Hasse ${ }^{138}\left(p^{s}, \rho^{\prime}, \rho^{\prime \prime}\right)$ & 2019 & 39 & $0.69-1.29$ \\
\hline this work $\left(p^{s}, \rho^{\prime}, \rho^{\prime \prime}, \Delta h_{v}\right)$ & 2019 & 124 & $0.69-1.28$ \\
\hline \multicolumn{4}{|l|}{ Vapor-liquid surface tension $\gamma$} \\
\hline Lee et al. ${ }^{98}$ & 1974 & 5 & $0.7-1.2$ \\
\hline Miyazaki et al. ${ }^{139}$ & 1976 & 1 & 0.7 \\
\hline Chapela et al. ${ }^{99}$ & 1977 & 5 & $0.7-1.27$ \\
\hline Nijmeijer et al. ${ }^{102}$ & 1988 & 1 & 0.92 \\
\hline Holcomb et al. ${ }^{105}$ & 1993 & 3 & $0.72-1.13$ \\
\hline Mecke et al. ${ }^{140}$ & 1997 & 3 & $0.7-1.1$ \\
\hline Guo et al. ${ }^{111}$ & 1997 & 5 & $0.75-1.25$ \\
\hline Guo and $L u^{112}$ & 1997 & 4 & $0.75-1.15$ \\
\hline Trokhymchuk and Alejandre ${ }^{114}$ & 1999 & 6 & $0.72-1.27$ \\
\hline Anisimov et al. ${ }^{115}$ & 1999 & 6 & $0.75-1$ \\
\hline Potoff and Panagiotopoulos ${ }^{116}$ & 2000 & 18 & $0.95-1.31$ \\
\hline Baidakov et al. ${ }^{117}$ & 2000 & 7 & $0.72-1.23$ \\
\hline Chen et al. ${ }^{24}$ & 2001 & 3 & $0.7-0.8$ \\
\hline Baidakov et al. ${ }^{123}$ & 2002 & 6 & $0.72-1.22$ \\
\hline Errington $^{125}$ & 2003 & 4 & $0.7-1.3$ \\
\hline Baidakov et al. ${ }^{129}$ & 2007 & 12 & $0.5-1.2$ \\
\hline Shen et al. ${ }^{141}$ & 2007 & 10 & $0.7-1.1$ \\
\hline Janeček et al. ${ }^{131}$ & 2009 & 4 & $0.72-1.25$ \\
\hline
\end{tabular}

Continued on next page 


\begin{tabular}{|c|c|c|c|}
\hline Authors & Year & $\#$ & $T$ \\
\hline Galliero et al. ${ }^{132}$ & 2009 & 13 & $0.7-1.3$ \\
\hline Galliero et al. ${ }^{132}$ & 2009 & 6 & $0.7-1.2$ \\
\hline Werth et al. ${ }^{142}$ & 2013 & 7 & $0.7-1.25$ \\
\hline Martinez-Ruiz et al. ${ }^{134}$ & 2014 & 7 & $0.7-1.1$ \\
\hline Janeček et al. ${ }^{135}$ & 2017 & 8 & $0.7-1.25$ \\
\hline Werth et al. ${ }^{137}$ & 2017 & 9 & $0.72-1.24$ \\
\hline Stephan and Hasse ${ }^{138}$ & 2019 & 13 & $0.69-1.29$ \\
\hline \multicolumn{4}{|l|}{ SLE } \\
\hline Hansen and Verlet ${ }^{20}$ & 1969 & 4 & $0.75-2.74$ \\
\hline Hansen $^{50}$ & 1970 & 6 & $2.74-100$ \\
\hline Agrawal and Kofke 106 & 1995 & 37 & $0.69-274$ \\
\hline van der Hoef ${ }^{143}$ & 2000 & corr. ${ }^{(A)}$ & $0.1-2.0$ \\
\hline Barroso and Ferreira ${ }^{144}$ & 2002 & 18 & $0.69-4.5$ \\
\hline Morris and Song ${ }^{145}$ & 2002 & 12 & $0.72-2.65$ \\
\hline Errington $^{146}$ & 2004 & 2 & $0.75-2$ \\
\hline McNeil-Watson and Wilding ${ }^{147}$ & 2006 & 34 & $0.72-83$ \\
\hline Mastny and Pablo ${ }^{148}$ & 2007 & 5 & $1-20$ \\
\hline Ahmed and Sadus ${ }^{149,150}$ & 2009 & 5 & $0.8-2.74$ \\
\hline Sousa et al. ${ }^{151}$ & 2012 & 10 & $0.75-5$ \\
\hline Köster et al. ${ }^{91}$ & 2017 & 8 & $1.3-30$ \\
\hline Schultz and Kofke $e^{95}$ & 2018 & $\operatorname{corr}^{(\mathrm{A})}$ & $0.68-2272$ \\
\hline \multicolumn{4}{|l|}{ Isochoric heat capacity $c_{v}$} \\
\hline Wood and Parker ${ }^{15}$ & 1957 & 11 & 2.74 \\
\hline McDonald and Singer ${ }^{45}$ & 1967 & 26 & $0.72-1.24$ \\
\hline McDonald and Singer ${ }^{19}$ & 1967 & 48 & $1.45-3.53$ \\
\hline Adams $^{57}$ & 1975 & 12 & $2-4$ \\
\hline Adams $^{60}$ & 1979 & 31 & $1.15-1.35$ \\
\hline Saager et al. ${ }^{152,153}$ & 1990 & 12 & $1.1-1.35$ \\
\hline Boda et al. ${ }^{154}$ & 1996 & 9 & $1.31-2$ \\
\hline Roccatano et al. ${ }^{79}$ & 1998 & 10 & $1.4-10$ \\
\hline Meier 80,155 & 2002 & 327 & $0.7-6$ \\
\hline Baidakov et al. ${ }^{83}$ & 2008 & 208 & $0.35-2$ \\
\hline May and Mausbach ${ }^{85,156}$ & 2012 & 218 & $0.68-6.17$ \\
\hline Yigzawe and Sadus ${ }^{86,87}$ & 2012 & 406 & $1.3-2.62$ \\
\hline Mairhofer and Sadus ${ }^{88,89}$ & 2013 & 282 & $1.36-3.05$ \\
\hline Thol et al. ${ }^{90}$ & 2016 & 197 & $0.7-9$ \\
\hline Köster et al. ${ }^{91}$ & 2017 & 45 & $1.01-30$ \\
\hline Köster et al. ${ }^{91,92}$ & 2017 & 65 & $1.01-30$ \\
\hline this work & 2019 & 515 & $0.7-90$ \\
\hline \multicolumn{4}{|l|}{ Isobaric heat capacity $c_{p}$} \\
\hline Boda et al. ${ }^{154}$ & 1996 & 41 & $0.65-1.9$ \\
\hline
\end{tabular}




\begin{tabular}{|c|c|c|c|}
\hline Authors & Year & $\#$ & $T$ \\
\hline Lustig $^{84}$ & 2011 & 6 & $1.02-3$ \\
\hline May and Mausbach 85,156 & 2012 & 202 & $0.69-6.17$ \\
\hline Yigzawe and Sadus ${ }^{86,87}$ & 2012 & 406 & $1.3-2.62$ \\
\hline Mairhofer and Sadus ${ }^{88,89}$ & 2013 & 282 & $1.36-3.05$ \\
\hline Thol et al. ${ }^{90}$ & 2016 & 197 & $0.7-9$ \\
\hline Köster et al. ${ }^{91}$ & 2017 & 45 & $1.01-30$ \\
\hline Köster et al. ${ }^{91,92}$ & 2017 & 65 & $1.01-30$ \\
\hline this work & 2019 & 515 & $0.7-90$ \\
\hline \multicolumn{4}{|l|}{ Grüneisen coefficient $\Gamma$} \\
\hline Emampour et al. ${ }^{157}$ & 2011 & 26 & $1.2-1.8$ \\
\hline Mausbach and May ${ }^{158}$ & 2014 & 212 & $0.69-6.17$ \\
\hline Thol et al. ${ }^{90}$ & 2016 & 197 & $0.7-9$ \\
\hline Mausbach et al. ${ }^{159}$ & 2016 & 110 & $0.72-9$ \\
\hline Köster et al. ${ }^{91}$ & 2017 & 45 & $1.01-30$ \\
\hline Köster et al. ${ }^{91,92}$ & 2017 & 65 & $1.01-30$ \\
\hline this work & 2019 & 515 & $0.7-90$ \\
\hline \multicolumn{4}{|c|}{ Thermal expansion coefficient $\alpha$} \\
\hline McDonald and Singer ${ }^{45}$ & 1967 & 20 & $0.72-1.24$ \\
\hline Adams $^{57}$ & 1975 & 12 & $2-4$ \\
\hline Yigzawe and Sadus ${ }^{86,87}$ & 2012 & 406 & $1.3-2.62$ \\
\hline Thol et al. ${ }^{90}$ & 2016 & 197 & $0.7-9$ \\
\hline Köster et al. ${ }^{91}$ & 2017 & 45 & $1.01-30$ \\
\hline Köster et al. ${ }^{91,92}$ & 2017 & 65 & $1.01-30$ \\
\hline this work & 2019 & 515 & $0.7-90$ \\
\hline \multicolumn{4}{|c|}{ Isothermal compressibility $\beta$} \\
\hline McDonald and Singer ${ }^{45}$ & 1967 & 22 & $0.72-1.24$ \\
\hline Adams $^{57}$ & 1975 & 12 & $2-4$ \\
\hline Adams $^{60}$ & 1979 & 31 & $1.15-1.35$ \\
\hline Lotfi et al. ${ }^{71}$ & 1992 & 19 & $0.7-1.3$ \\
\hline Lustig $^{77}$ & 1994 & 2 & 1.18 \\
\hline Morsali et al. ${ }^{82}$ & 2007 & 13 & 3.76 \\
\hline May and Mausbach 85,156 & 2012 & 205 & $0.69-6.17$ \\
\hline Yigzawe and Sadus ${ }^{86,87}$ & 2012 & 406 & $1.3-2.62$ \\
\hline Mairhofer and Sadus ${ }^{88,89}$ & 2013 & 282 & $1.36-3.05$ \\
\hline Thol et al. ${ }^{90}$ & 2016 & 197 & $0.7-9$ \\
\hline Köster et al. ${ }^{91}$ & 2017 & 45 & $1.01-30$ \\
\hline Köster et al. ${ }^{91,92}$ & 2017 & 65 & $1.01-30$ \\
\hline this work & 2019 & 515 & $0.7-90$ \\
\hline \multicolumn{4}{|c|}{ Thermal pressure coefficient $\gamma$} \\
\hline McDonald and Singer ${ }^{45}$ & 1967 & 20 & $0.72-1.24$ \\
\hline
\end{tabular}




\begin{tabular}{|c|c|c|c|}
\hline Authors & Year & $\#$ & $T$ \\
\hline $\operatorname{Adams}^{57}$ & 1975 & 12 & $2-4$ \\
\hline Lustig $^{77}$ & 1994 & 2 & 1.18 \\
\hline Meier $^{80}$ & 2002 & 326 & $0.7-6$ \\
\hline Morsali et al. ${ }^{82}$ & 2007 & 13 & 3.76 \\
\hline May and Mausbach ${ }^{85,156}$ & 2012 & 205 & $0.69-6.17$ \\
\hline Yigzawe and Sadus ${ }^{86,87}$ & 2012 & 406 & $1.3-2.62$ \\
\hline Mairhofer and Sadus ${ }^{88,89}$ & 2013 & 282 & $1.36-3.05$ \\
\hline Thol et al. ${ }^{90}$ & 2016 & 197 & $0.7-9$ \\
\hline Köster et al. ${ }^{91}$ & 2017 & 45 & $1.01-30$ \\
\hline Köster et al. ${ }^{91,92}$ & 2017 & 65 & $1.01-30$ \\
\hline this work & 2019 & 515 & $0.7-90$ \\
\hline \multicolumn{4}{|l|}{ Speed of sound $w$} \\
\hline Meier 80 & 2002 & 349 & $0.7-6$ \\
\hline Lustig $^{84}$ & 2011 & 8 & $1.02-3$ \\
\hline May and Mausbach ${ }^{85,156}$ & 2012 & 205 & $0.69-6.17$ \\
\hline Yigzawe and Sadus ${ }^{86,87}$ & 2012 & 406 & $1.3-2.62$ \\
\hline Mairhofer and Sadus ${ }^{88,89}$ & 2013 & 282 & $1.36-3.05$ \\
\hline Thol et al. ${ }^{90}$ & 2016 & 197 & $0.7-9$ \\
\hline Köster et al. ${ }^{91}$ & 2017 & 45 & $1.01-30$ \\
\hline Köster et al. ${ }^{91,92}$ & 2017 & 65 & $1.01-30$ \\
\hline this work & 2019 & 515 & $0.7-90$ \\
\hline \multicolumn{4}{|c|}{ Joule-Thomson coefficient $\mu_{\text {JT }}$} \\
\hline Lustig $^{84}$ & 2011 & 8 & $1.02-3$ \\
\hline May and Mausbach ${ }^{85,156}$ & 2012 & 205 & $0.69-6.17$ \\
\hline Yigzawe and Sadus ${ }^{86,87}$ & 2012 & 406 & $1.3-2.62$ \\
\hline Mairhofer and Sadus ${ }^{88,89}$ & 2013 & 282 & $1.36-3.05$ \\
\hline Thol et al. ${ }^{90}$ & 2016 & 197 & $0.7-9$ \\
\hline Köster et al. ${ }^{91}$ & 2017 & 45 & $1.01-30$ \\
\hline Köster et al. ${ }^{91,92}$ & 2017 & 65 & $1.01-30$ \\
\hline this work & 2019 & 515 & $0.7-90$ \\
\hline \multicolumn{4}{|l|}{ Entropic properties $a, \mu$} \\
\hline Levesque and Verlet ${ }^{48}(a)$ & 1969 & 8 & 1.35 \\
\hline Hansen and Verlet $^{20}(a)$ & 1969 & 25 & $0.75-1.15$ \\
\hline Weeks et al. ${ }^{53}(a)$ & 1971 & 5 & $0.75-1.35$ \\
\hline Weeks et al. ${ }^{160}(a)$ & 1971 & 27 & $0.75-1.35$ \\
\hline $\operatorname{Adams}^{57}(\mu)$ & 1975 & 12 & $2-4$ \\
\hline Torrie and Valleau ${ }^{96}(a)$ & 1977 & 16 & $0.75-2.74$ \\
\hline $\operatorname{Adams}^{60}(\mu)$ & 1979 & 31 & $1.15-1.35$ \\
\hline Yao et al. ${ }^{63}(\mu)$ & 1982 & 12 & $1.15-1.25$ \\
\hline Powles et al. ${ }^{64}(\mu)$ & 1982 & 37 & $0.7-1.41$ \\
\hline Panagiotopoulos et al. ${ }^{101}(\mu)$ & 1988 & 18 & $0.75-1.3$ \\
\hline
\end{tabular}




\begin{tabular}{|c|c|c|c|}
\hline Authors & Year & $\#$ & $T$ \\
\hline Baranyai and Evans ${ }^{68}(a)$ & 1989 & 6 & 1.15 \\
\hline Lotfi et al. ${ }^{71}(\mu)$ & 1992 & 19 & $0.75-1.3$ \\
\hline $\operatorname{Han}^{161}(\mu)$ & 1992 & 3 & 1.2 \\
\hline Kolafa et al. ${ }^{74}(\mu)$ & 1993 & 7 & $1.2-1.45$ \\
\hline Lustig $^{77}(\mu)$ & 1994 & 2 & 1.18 \\
\hline Cuadros et al. ${ }^{162}(a)$ & 1996 & 269 & $0.7-2.6$ \\
\hline Hong and Jhon ${ }^{163}(a)$ & 1997 & 36 & $0.59-2.89$ \\
\hline Hong and Jang ${ }^{164}(a)$ & 2003 & 22 & $0.59-2.85$ \\
\hline Thol et al. ${ }^{90}(a, \mu)$ & 2016 & 197 & $0.7-9$ \\
\hline Köster et al. ${ }^{91,92}(a, \mu)$ & 2017 & 65 & $1.01-30$ \\
\hline Ustinov $^{93,94}(\mu)$ & 2017 & 232 & $0.76-1.14$ \\
\hline this work $(a, \mu)$ & 2019 & 655 & $0.7-90$ \\
\hline \multicolumn{4}{|l|}{ Virial coefficients $B, C$} \\
\hline Bird et al. ${ }^{165}(C)$ & 1950 & 74 & $0.7-400$ \\
\hline Hirschfelder et al. ${ }^{166}(B, C)$ & 1954 & 156 & $0.3-400$ \\
\hline Barker et al. ${ }^{167}(B, C)$ & 1966 & 66 & $0.625-20$ \\
\hline Nicolas et al. ${ }^{61}(B)$ & 1979 & 33 & $0.625-20$ \\
\hline Sun and Teja ${ }^{168}(B, C)$ & 1996 & 302 & $0.39-6.1$ \\
\hline Shaul et al. ${ }^{169}(B, C)$ & 2010 & 22 & $0.7-2$ \\
\hline Wheatley ${ }^{170,171}(B, C)$ & 2013 & 100 & $0.1-1000$ \\
\hline \multicolumn{4}{|l|}{ Ideal curves ID, BL, JTI, JI ${ }^{(\mathrm{B})}$} \\
\hline Heyes and Llaguno ${ }^{172}(\mathrm{JTI})$ & 1992 & 16 & $1.15-7.1$ \\
\hline Colina and Müller ${ }^{173}$ (JTI) & 1999 & 24 & $1.12-6.4$ \\
\hline Kioupis et al. ${ }^{124}$ (JTI) & 2002 & 29 & $1.3-6$ \\
\hline Vrabec et al. ${ }^{174}$ (JTI) & 2005 & 18 & $1.2-6.3$ \\
\hline Yigzawe and Sadus ${ }^{87}(\mathrm{JTI})$ & 2013 & 9 & $1.31-2.62$ \\
\hline Deiters and Neumaier ${ }^{43,97}$ (ID, BL, JTI, JI) & 2016 & 43 & $0.01-6.4$ \\
\hline
\end{tabular}

(A) The authors provided no numerical data, but a correlation that was parametrized to computer experiment data.

(B) ID: Classical ideal curve, BL: Boyle curve, JTI: Joule-Thomson inversion curve, JI: Joule inversion curve.

The simulation method proposed by Lustig ${ }^{84,175}$ provides Helmholtz energy derivatives with respect to the density and the inverse temperature simultaneously from a single simulation run. This technique can be combined with Widom's test particle insertion method ${ }^{176}$ for the determination of the Helmholtz energy itself. This approach was applied to the Lennard-Jones fluid by Thol et al. ${ }^{90}$ and Köster et al. ${ }^{91,92}$ in different fluid regions. The simulations of Thol et al. ${ }^{90}$ were 
restricted to stable fluid states below $T=9$, while the simulation data of Köster et al. ${ }^{91,92}$ focused on the high density region close to the freezing line. These two data sets are complemented in the present work by data on 655 additional homogeneous state points, cf. Figure. 1, that were also sampled with the Lustig formalism. The new data was taken in order to get more information on regions in which literature data was comparatively sparse. For the metastable region, only pressure, chemical potential, and internal energy are reported here. The entity of the simulation results of Refs. ${ }^{90-92}$ and from this work is referred to as the Lustig formalism (LF) data set in the following. All of them were sampled with the simulation program $m s 2^{177}$ using similar simulation settings. Details on the simulation procedure are given in Appendix .

Results on VLE properties of the Lennard-Jones fluid have been reported many times in the literature, cf. Table 1. 45 VLE data sets were found in the progress of this work. The simulation methods used in these studies can be separated in two types: simulations in which the liquid and the vapor phase are simulated in two separate volumes that are suitably coupled and simulations in which the liquid and the vapor phase coexist in a single volume that also contains the interface. The first are referred to as indirect methods and the latter as direct methods in the following. Both VLE simulation types provide in general vapor pressure $p^{s}$, saturated densities of the liquid and vapor phase $\rho^{\prime}$ and $\rho^{\prime \prime}$, respectively, and enthalpy of vaporization $\Delta h_{v}$ at a given temperature T. However, often only a subset of these properties was reported in the literature, cf. Table 1. Simulation techniques that belong to the indirect type ${ }^{178}$ are the Gibbs ensemble method, ${ }^{100,101}$ the Grand equilibrium method, ${ }^{179}$ the $N p T$ +test particle method, ${ }^{180}$ Gibbs-Duhem integration, ${ }^{181}$ and the Wang-Landau method. ${ }^{182}$ Additional information on interfacial properties can be obtained with the direct method. ${ }^{183,184}$ Data on the surface tension of the Lennard-Jones fluid are also included in the database, cf. Table 1. The Grand equilibrium method was employed in the present work to obtain an extended VLE data set for the Lennard-Jones fluid. Details are given in Appendix .

Data on the triple point ${ }^{20,24,95,106,144,148-151,185-188}$ and the critical point ${ }^{18,46,48,60,61,70,71,73,75,78,100,104,107,113,1}$ of the Lennard-Jones fluid have been reported many times. Numeric values of the critical point are summarized in Table 2 . The critical pressure has been less frequently reported than the critical temperature and density. The critical parameters of the LJ fluid reported in the literature scatter 
over a large range - especially regarding the temperature. However, 13 critical temperature and density data points (Refs. ${ }^{71,104,119,120,122,191,197,198,200,202}$ ) are in fairly good agreement, even though most of them do not agree within their combined statistical uncertainties. The critical temperature reported by Refs. ${ }^{71,104,119,120,122,191,197,198,200,202}$ scatters in a range of $T_{c}=1.31 . .1 .326$; the critical density in a range of $\rho_{c}=0.304 . .0 .318$. The reported critical densities obtained from computer experiment reported by Refs. ${ }^{71,104,119,120,122,191,198,200,202}$ cluster around two different values: $\rho_{c}=0.305$ and 0.316 . Other data points were discarded since they significantly deviate from this entity. The critical pressure reported by Refs. ${ }^{60,119,122,192,197}$ scatters in the range $p_{c}=0.125 . .0 .135$.

In the following, the critical point is presumed to be located at $T_{c}=1.321 \pm 0.007, \rho_{c}=0.316 \pm 0.005$ and $p_{c}=0.129 \pm 0.005$. The stated uncertainties were estimated from the standard deviation of the critical parameters reported by Refs. ${ }^{71,104,119,120,122,191,198,200,202}$ for the critical temperature and density and Refs. ${ }^{60,119,122,192,197}$ for the critical pressure.

The solid-fluid equilibrium of the Lennard-Jones fluid is used in this study only to delimit the fluid region, as molecular simulation results beyond the freezing line were excluded regarding the assessment. Solid-fluid equilibria have been investigated multiple times, cf. Table 1. The correlation for the freezing line by Köster et al. ${ }^{91}$ was employed here.

\section{Database of thermophysical properties}

All thermophysical property data (approximately 35,000 data points) considered in this work are summarized in a consistent form in an .xls spreadsheet in the electronic supplementary material of this work. All data are sorted by thermophysical properties in that spreadsheet. All numerical values are given in a consistent form, i.e. as residual properties with respect to the ideal gas and in the standard 'Lennard-Jones' units. Information on the statistical uncertainties were adopted from publications - wherever such information was reported, which is unfortunately not always the case. The methods for the estimation of statistical uncertainties differ significantly among the publications. Sometimes, statistical uncertainties were reported, but no description on how such were obtained is given. Due to this heterogeneity, statistical uncertainties could unfortunately not 
be used for the assessment of data points in this work.

The database furthermore contains notes on pitfalls regarding the conversion of the primary literature data to the format used in the database. Furthermore, known misprints in publications from the literature were corrected, e.g. ${ }^{90,92,155}$ A survey of these misprints is also given. Each data point possesses an additional mark, which indicates whether the data point was found to be an outlier or not, according the assessment described in the following.

\section{Assessment of molecular simulation data}

\section{Assessment of data on homogeneous states}

Thermophysical property data of the Lennard-Jones fluid reported in the literature (see Table 1) for homogeneous state points were assessed by an equation of state (EOS) consistency test. This

consistency test is based on the statistical method proposed by Rousseeuw and Croux ${ }^{206}$ for the detection of outliers that was adopted and extended for the purposes of the present work. The EOS test evaluates each data point individually by comparing it with the most accurate equations of state of the Lennard-Jones fluid available in the literature and computer experiment data in its vicinity. The EOS test is designed in a way to make a binary decision: either a data point is an outlier or not.

The relative deviation $\delta x$ of a given data point $(\mathrm{DP}) x_{\mathrm{DP}}(T, \rho)$ from a given EOS at the same temperature and density was computed as

$$
\delta x=\frac{x_{\mathrm{DP}}-x_{\mathrm{EOS}}}{x_{\mathrm{EOS}}},
$$

where $x$ is one of the following homogeneous bulk phase properties: pressure $p$, internal energy $u$, speed of the sound $w$, thermal pressure coefficient $\gamma$, Grüneisen coefficient $\Gamma$, thermal expansion coefficient $\alpha$, isothermal compressibility $\beta$, Joule-Thomson coefficient $\mu_{\mathrm{JT}}$, isochoric heat capacity $c_{v}$, isobaric heat capacity $c_{p}$, Helmholtz energy divided by the temperature $\tilde{a}=a / T$, or chemical potential $\mu$. 
Comprehensive comparisons of EOS for the Lennard-Jones fluid have been carried out recently. ${ }^{90,207}$ Six of the most accurate EOS were used here: Johnson et al. ${ }^{73}$ Kolafa and Nezbeda, ${ }^{76}$ Lafitte et al., ${ }^{11}$ Mecke et al., ${ }^{78,208}$ Stephan et al. ${ }^{207}$ and Thol et al. ${ }^{90}$ (alphabetically). However, also these EOS have deficiencies, e.g. the EOS of Thol et al. ${ }^{90}$ exhibits an unrealistic behavior in the two-phase region, that of Mecke et al. ${ }^{78,208}$ shows large deviations in the high density region close to the freezing line, that of Stephan et al. ${ }^{207}$ is less accurate in the high temperature region, the EOS of Kolafa and Nezbed ${ }^{76}$ produces distorted characteristic curves, ${ }^{43}$ and the one of Lafitte et al. ${ }^{11}$ is less accurate in the low density gas region. The EOS from Johnson et al. ${ }^{73}$ is overall less precise than the ones mentioned before. To take these deficiencies into account in evaluating an individual data point, only the four EOS were considered that yielded the smallest relative deviation $\delta x_{j}$ for a given data point. The four best EOS were identified and selected for each data point individually, to prevent that the mentioned deficiencies of the used EOS adulterate the assessment.

To take into account that the precision of molecular simulation results varies in different regions, the deviation of each individual state point was put into relation with the average deviation of the data points in its neighborhood in the $T-\rho$ plane. Each data point $j$ has its own neighborhood with $i=1 . . M$ neighbors, where the number of neighbors is $M=15 . .20$. The nearest neighbors were determined using the radial distance $\tilde{\delta} R$ between data points in the $T-\rho$ plane, i.e. $\tilde{\delta} R=$ $\left(\tilde{\delta} T^{2}+\tilde{\delta} \rho^{2}\right)^{0.5}$. The choice of the radius and, hence, of $M$ depends on the location of the data point in the $T-\rho$ plane, i.e. in a fluid region where data points are sparse or if a data point is close to a phase boundary the radius was chosen large enough, such that at least 15 neighbors were allocated to the neighborhood of each data point. The neighbors of each data point $j$ were taken from the entity of all data points $N$ of a given property, i.e. the neighborhood $M$ contains in general data points from different publications.

To decide whether a data point $j$ is an outlier, a measure $P_{j}$ is introduced. Following the ideas of Refs., ${ }^{38,39,206,209} P_{j}$ was defined as

$$
P_{j}=\frac{\left|\delta x_{j}-\operatorname{median}_{i=1}^{M}\left(\delta x_{j i}\right)\right|}{\operatorname{MAD}_{j}} \quad \text { with } i=1 . . M \text { and } j=1 . . N
$$


In the measure $P_{j}$, the deviation of a tested data point $j$ and an EOS are compared to the deviations of the data points from its neighborhood and that EOS. Details on the selection of the EOS are given below. The numerator on the right hand side of Eq. (3) is the absolute deviation of the data point $j$ and the median ${ }^{210}$ of the data points in its neighborhood. The denominator is a measure for the mean absolute deviation (MAD) of the data points in the neighborhood of the given data point $^{211} j$

$$
\mathrm{MAD}_{j}=k \cdot \operatorname{median}_{i=1}^{M}\left(\left|\delta x_{i j}-\operatorname{median}_{i=1}^{M}\left(\delta x_{i j}\right)\right|\right),
$$

where $k=1.4826$. The selection of that number is related to the assumption that the deviation data $\delta x_{i j}$ is normally distributed in the set of data points. ${ }^{212}$ The $\mathrm{MAD}_{j}$ quantifies how strong the data points of the neighborhood $M$ scatter on average from their median, i.e. how precise the data in the neighborhood is on average. The MAD is robust, as the median is less affected by outliers than the mean. Hence, this test takes the accuracy of the EOS and the accuracy of the computer experiment data of a certain thermophysical property in a certain fluid region into account. For brevity, it is called EOS test in the following.

The EOS test requires a sufficiently dense neighborhood of data points, to ensure that the accuracy of the reference, i.e. the EOS, remains fairly constant within the neighborhood. Since the data points for $T>6$ are sparsely distributed in the $T-\rho$ plane, the EOS test was only applied to data at $T \leqslant 6$.

The measure $P_{j}$ was computed for the four EOS that were selected as described above, and then compared with a parameter $P_{\max }$

$$
P_{j} \geqslant P_{\max }
$$

If this decision criterion was fulfilled for at least two of the four EOS, the data point $j$ was identified as an outlier. The parameter $P_{\max }$ introduces an unavoidable subjective attribute into the method 206,209 and regulates the severity of the EOS test. A fixed number for $P_{\max }$ is used here for all thermophysical properties, EOS, and fluid regions under consideration, which established a consistent framework. The parameter was set to $P_{\max }=4$. This results in a confirmation rate of approximately $90 \%$ of all homogeneous data. Vise versa, $10 \%$ of all homogeneous data points 
were identified as outliers. This rather conservative choice ${ }^{206,209}$ for $P_{\max }$ was used to prevent a false assessment of data points to be erroneous. A more stringent, but smaller database can be generated easily by increasing $P_{\max }$ and vice versa a larger database, that might however include less precise data, can be generated by decreasing $P_{\max }$. The EOS test enables a robust assessment of the quality of individual data points based on an assessment of the precision of the data points in its neighborhood.

Figure. 2 summarizes the results of the EOS test for the homogeneous state points for $P_{\max }=4$. About $85 \%$ of the $p v T$ data and the data for the internal energy $u$ passed the EOS test. These two thermophysical properties are the most frequently reported: the database contains about ten times more $p v T$ and internal energy data than any other thermophysical property. The relatively low confirmation rate of the $p v T$ and internal energy data is a result from significant differences in the precision among different publications that the EOS test used for the detection of the outliers.

Confirmation rates of $94-96 \%$ were obtained for data for the isochoric heat capacity $c_{v}$, isobaric heat capacity $c_{p}$, speed of sound $w$, thermal pressure coefficient $\gamma$, Joule-Thomson coefficient $\mu_{\mathrm{JT}}$, and Grüneisen coefficient $\Gamma$ - which are higher order temperature and density derivatives of the Helmholtz energy. The confirmation rate for the thermal expansion coefficient $\alpha$ and isothermal compressibility $\beta$ was $92-93 \%$. A confirmation rate of $89 \%$ was found for the chemical potential $\mu$, which is particularly challenging to determine by molecular simulation. The lowest confirmation rate of $72 \%$ was found for the Helmholtz energy $\tilde{a}$, which is mainly due to particularly low confirmation rates for the results from some publications. Details are given in the supplementary material.

Also, the numerical values of all data points and the corresponding result of the EOS test, i.e. whether a data point is confirmed or identified as an outlier with $P_{\max }=4$, are provided in the supplementary material. Moreover, information on the dependence of the total percentage of confirmed data on the choice of the number for $P_{\max }$ is presented there. 


\section{Assessment of vapor-liquid equilibrium data}

For the vapor-liquid equilibrium, both bulk and interfacial properties were considered: vapor pressure $p^{s}$, saturated liquid and vapor density $\rho^{\prime}$ and $\rho^{\prime \prime}$, and enthalpy of vaporization $\Delta h_{v}$ were considered for the bulk properties and the interfacial tension $\gamma$ as interfacial property, cf. Table 1.

\section{Bulk VLE data}

In contrast to the test on the homogeneous state points, which was assessed for each data point individually entire VLE bulk property data sets from a given publication were assessed and either confirmed or discarded. Furthermore, the EOS test for homogeneous data is designed to identify gross outliers, whereas the assessment of the VLE data additionally aims at determining the most precise data and to give an estimation of that precision. The estimated precision indicates the magnitude that a given data set scatters around the presumed true value. The true value, i.e. exact and correct, of a given property is approximated here by the mean of the most precise and accurate data sets.

VLE bulk data of the Lennard-Jones fluid were assessed by several independent consistency tests. Two of them were taken from the literature: the compressibility factor test proposed by Nezbeda $a^{213,214}$ and the Clausius-Clapeyron test. ${ }^{71}$ Furthermore, as a third test, outliers are determined from a direct comparison of the data points as described below in more detail. For brevity, this test is referred to as deviation test in the following. Data sets were discarded, if the majority of data points within a data set violate one or more consistency tests. For the following discussion, the vicinity to the critical point of the VLE region is defined to be above $95 \%$ of the critical temperature. Data points in that region were not included in the consistency tests.

To facilitate the assessment, empirical correlation functions for the vapor pressure $p^{s}$, saturated 
liquid and vapor density $\rho^{\prime}$ and $\rho^{\prime \prime}$, and enthalpy of vaporization $\Delta h_{v}$ were used:

$$
\begin{aligned}
\ln p^{s} & =n_{1} T+\frac{n_{2}}{T}+\frac{n_{3}}{T^{n_{4}}}, \\
\left(\frac{\rho^{\prime}}{\rho_{\mathrm{c}}}\right) & =1+\sum_{i=1}^{5} n_{i}\left(1-\frac{T}{T_{\mathrm{c}}}\right)^{t_{i}}, \\
\ln \left(\frac{\rho^{\prime \prime}}{\rho_{\mathrm{c}}}\right) & =\sum_{i=1}^{5} n_{i}\left(1-\frac{T}{T_{\mathrm{c}}}\right)^{t_{i}}, \\
\Delta h_{v} & =\sum_{i=1}^{4} n_{i}\left(T_{\mathrm{c}}-T\right)^{t_{i}} .
\end{aligned}
$$

In an initial step, the VLE data set from this work was used for the parametrization of Eqs. (6) - (9), as it is the most extensive data set. The absolute average deviation from the correlations (6) - (9) and the respective VLE data from this work is: $0.3 \%$ for the vapor pressure, $0.06 \%$ for the saturated liquid density, $0.5 \%$ for the saturated vapor density, and $0.3 \%$ for the enthalpy of vaporization. The numerical values of the parameters $n_{i}$ and $t_{i}$ are listed in Table 3 . The critical values used in Eqs. (6) - (9) are $T_{\mathrm{c}}=1.321$ and $\rho_{\mathrm{c}}=0.316$. They are called base correlations in the following. It will be shown below, that these correlations match the best available guess for the considered properties within their estimated precision.

In the test proposed by Nezbeda, ${ }^{213,214}$ the compressibility factor of the saturated vapor phase $Z^{\prime \prime}=p^{s} / \rho^{\prime \prime} T$ is considered. Starting at low temperatures close to the triple point, $Z^{\prime \prime}$ must be close to unity. With increasing temperature, $Z^{\prime \prime}$ must decrease monotonically until the compressibility factor at the critical point is reached. ${ }^{213,214}$

This simple criteria can be applied for sorting out outliers: $Z^{\prime \prime}>1$ is not acceptable and the slope $\mathrm{d} Z^{\prime \prime} / \mathrm{d} T$ must be negative. More generally, the slope $\mathrm{d} Z^{\prime \prime} / \mathrm{d} T$ is sensitive and useful for discriminating data points that deviate from the general trend. Furthermore, the data points were compared to the results for $Z^{\prime \prime}$ that was obtained from the base correlations. Data points for which the deviation in $Z^{\prime \prime}$ from that correlation is above $1.25 \%$ were considered as outliers. The number $1.25 \%$ was chosen on the basis of an examination of the scattering of the entire available data. Data sets of which more than $50 \%$ of the data points violate at least one of these criteria were discarded. The other data sets are considered as confirmed by the compressibility factor test. 
Figure. 3 shows the data sets that were confirmed by the compressibility factor test. These data are in good mutual agreement and in agreement with the base correlation computed from Eqs. (6) and (8). Excellent mutual agreement is found for the data sets from Agrawal and Kofke, ${ }^{106}$ Errington, ${ }^{125}$ Errington, ${ }^{126,127}$ Janeček et al.,${ }^{135,136}$ Lotfi et al.,${ }^{71}$ Mick et al.,${ }^{23}$ Okumura and Yonezawa, ${ }^{121,122}$ and from the present work. Taking these data as the reference, and their scattering as a measure for the precision, with which $Z^{\prime \prime}$ is known, that precision is estimated to be approximately $\pm 0.5 \%$ excluding the vicinity of the critical point. Furthermore, the data sets from Hansen and Verlet, ${ }^{20}$ Janeček ${ }^{131}$ Kofke,${ }^{104}$ Martínez-Ruiz et al.,${ }^{134}$ Okumura and Yonezawa, ${ }^{118,119}$ Stephan and Hasse, ${ }^{138}$ Stoll et al., ${ }^{128}$ and Werth et al. ${ }^{137}$ also agree well with the data mentioned above, but scatter more. That data were confirmed by the compressibility factor test. The larger scatter of the data of Refs. ${ }^{134,137,138}$ is probably due to the fact that these data were sampled with the direct simulation method. The data sets from Refs. ${ }^{118,119,125-127}$ each contain a single data point that is a clear outlier. These data points are explicitly pointed out in the supplementary information. However, apart from the outlier, these data sets are considered as confirmed by the compressibility factor test. Data sets from Refs. ${ }^{58,60,100,101,103,107,108,110,113-115,117,124,129,130}$ were discarded by the compressibility factor test. Details are given in the supplementary material.

The second consistency test that was employed for the VLE bulk data is based on the ClausiusClapeyron equation

$$
\frac{\mathrm{d} \ln p^{s}}{\mathrm{~d}(1 / T)}=-\frac{T \Delta h_{v}}{p^{s}\left(1 / \rho^{\prime \prime}-1 / \rho^{\prime}\right)}
$$

The left hand side (LHS) and right hand side (RHS) of Eq. (10) is considered individually for each data set and compared. The equality of the LHS and RHS within the corresponding statistical uncertainties indicates the thermodynamic consistency by the Clausius-Clapeyron equation. The RHS of Eq. (10) is therefor computed directly from the numerical values of the computer experiments. The statistical uncertainties of the RHS values are determined using the error propagation law. The LHS of Eq. (10) is calculated with an analytical function for the vapor pressure curve. The Clausius-Clapeyron test is applied here in two ways (Test A and Test B). In Test A, the LHS and RHS of a given data set are directly compared. This can only be done in a meaningful way 
for those data sets, that enable the parametrization of a correlation of $p^{s}(T)$ for the LHS in a sufficiently accurate manner. In Test B, the RHS of remaining data sets are compared with the LHS correlations established in Test A. Data points violate the Clausius-Clapeyron test in Test A, if the computed RHS of a given data point does not agree within the statistical uncertainty with the corresponding LHS-value. In the Test B, data points were considered as outliers, if they deviate $2 \%$ or more from the two correlations established in Test A. As for the compressibility factor test, data sets for which more than half of the data points violate the Clausius-Clapeyron test were discarded. The remaining data sets were considered as confirmed by the Clausius-Clapeyron test.

The data sets from Lotfi et al. ${ }^{71}$ and this work were employed in Test A. Correlations for $p^{s}(T)$ obtained from the other data sets were not found to be sufficiently accurate for carrying out a meaningful test of type A. For the data from this work, the base correlation for $p^{s}(T)$ from Eq. (6) was used for the comparison. The statistical uncertainty of the LHS was estimated from the error propagation law. Lotfi et al. ${ }^{71}$ published a correlation of their own $p^{s}$-results that was used here for computing the LHS of Eq. (10) for the comparison of the corresponding RHS-values.

Figure. 4 shows the VLE data sets that were confirmed by both the Clausius-Clapeyron test and the compressibility factor test. In the plot, results for the RHS of Eq. (10) that were obtained from the simulation data and are shown as symbols are compared with results for the LHS that were obtained from correlations of the vapor pressure curve and are shown as lines. Two such correlations are shown: one obtained from the data from the present work and one obtained by Lotfi et al. from their data. ${ }^{71}$ Lotfi et al. ${ }^{71}$ compared their results for the Lennard-Jones fluid with results for $\mathrm{Ne}, \mathrm{Ar}$, and $\mathrm{CH}_{4}$ and found very good qualitative agreement, i.e. a parabolic shape of $\mathrm{d}\left(\ln p^{s}\right) / \mathrm{d}\left(T^{-1}\right)$.

Figure. 4 shows that the two correlations of the LHS of Eq. (10) match well. The differences are far below the uncertainties of the data points for the RHS of Eq. (10). The data from the present work and from Lotfi et al. ${ }^{71}$ agree well with the correlations. There is one outlier in the data set from the present work. (It was not removed from the data set to ensure a fair comparison with the literature data, for which almost every data set was found to have at least one outlier). The LHS and RHS of Eq. (10) agree within the statistical uncertainties for all but this state point, 
which is likely the result of an overly optimistic error estimation. However, the data of Lotfi et al. ${ }^{71}$ and this work agree very well throughout, but the data of Lotfi et al. ${ }^{71}$ show a more pronounced scatter and larger error bars than the data from this work.

The RHS data of Janeček et al., ${ }^{135,136}$ Stoll et al., ${ }^{128}$ Mick et al., ${ }^{23}$ and Agrawal and Kofke ${ }^{106}$ are also in excellent agreement with the base correlation of the LHS of Eq. (10). The agreement is also very good for the data sets of Hansen and Verlet,${ }^{20}$ Janeček, ${ }^{131}$ Kofke, ${ }^{104}$ and Werth et al. ${ }^{137}$ but their RHS data exhibit considerable scatter, as does the data set of Lotfi et al. ${ }^{71}$

The data sets from Refs. ${ }^{58,60,100,101,103,108,110,124,130}$ were discarded by the Clausius-Clapeyron test. Details are given in the supplementary material. The results from the Clausius-Clapeyron test reinforce the findings from the compressibility factor test.

As a third assessment, the deviation plots of each VLE bulk property $\left(p^{s}, \rho^{\prime}, \rho^{\prime \prime}\right.$, and $\left.\Delta h_{\mathrm{v}}\right)$ from the base correlations were used as follows: First, the data sets with the best mutual agreement were identified, that were also confirmed by the previous two tests. From these data sets, a confidence interval $\pm X \%$ was estimated for each VLE property $\left(X=p^{s}, \rho^{\prime}, \rho^{\prime \prime}\right.$, and $\left.\Delta h_{\mathrm{v}}\right)$ individually, that is a measure for the precision of the available information. A data point was considered as an outlier, if its relative deviation to the corresponding base correlation exceeds 2.5 times the number of $\mathrm{X}$ of one of the four VLE properties. Again, data sets for which more than half of the data points of a given VLE property violate the criteria were discarded. The remaining data sets were considered as confirmed.

Figure. 5 shows the relative deviation of the VLE data (vapor pressure $p^{s}$, enthalpy of vaporization $\Delta h_{v}$, and the saturated densities $\rho^{\prime}$ and $\rho^{\prime \prime}$ ) from the base correlations. Excellent mutual agreement (and also agreement with the base correlations) was found for the data sets from Errington, ${ }^{126,127}$ Errington, ${ }^{125}$ Janeček et al.,${ }^{135,136}$ Lotfi et al.,${ }^{71}$ Mick et al.,${ }^{23}$ Okumura and Yonezawa ${ }^{121,122}$ and the data set from the present work. These seven data sets were also confirmed by the other two tests and comprise a total of 86 state points. Interestingly, the precision, i.e. the scatter, of each of the seven best data sets (Refs. ${ }^{23,71,121,122,125-127,135,136}$ and this work) is very similar. Only the saturated vapor density and vapor pressure data of Errington ${ }^{126,127}$ were found to be significantly more precise than the other data sets. However, the scatter of the saturated 
liquid density data of Errington ${ }^{126,127}$ is significantly larger than the scatter of the other selected best data sets (Refs. ${ }^{23,71,121,122,125-127,135,136}$ and this work).

The mutual agreement of these seven selected best bulk VLE data sets is approximately $\pm 1 \%$ for the vapor pressure, $\pm 0.2 \%$ for the saturated liquid density, $\pm 1 \%$ for the saturated vapor density, and $\pm 0.75 \%$ for the enthalpy of vaporization - excluding the region close to the critical point. Five of these seven best data sets contain a single or two clear outliers, which are listed in the supplementary material.

The mutual agreement of the seven selected best data sets (Refs. ${ }^{23,71,121,122,125-127,135,136}$ and the data set from the present work) significantly decreases in the region close to the critical point, i.e. $T \gtrsim 1.25$. The mutual agreement in the vicinity of the critical point is better for the vapor pressure and the saturated liquid density than for the enthalpy of vaporization and the saturated vapor density.

The following data sets agree well but scatter strongly (however, within the confidence interval and are thereby considered as confirmed by the deviation test): Betancourt-Cárdenas et al., ${ }^{130}$ Chen et al.,${ }^{24}$ Hansen and Verlet, ${ }^{20}$ Martinez-Ruiz et al.,${ }^{134}$ Okumura and Yonezawa, ${ }^{118,119}$ Stoll et al., ${ }^{128}$ Stephan and Hasse, ${ }^{138}$ and Werth et al. ${ }^{137}$ The data sets from Refs. ${ }^{58,60,98-115,117,123,124,129,131-133,135,197}$ were discarded because more than $50 \%$ of the respective data points exceed the confidence interval of one of the bulk VLE properties.

The seven data sets from Refs. ${ }^{23,71,121,122,125-127,135,136}$ and from the present work were confirmed by all applicable tests and were found to be in excellent mutual agreement and are therefore recommended as a reference. The data sets from Refs. ${ }^{20,118,119,128,134,137,138}$ were also confirmed by all applicable tests, but exhibit a significantly larger scattering compared to these seven most precise data sets. The data sets from Refs. ${ }^{24,98,102,105-107,111,112,116,120,123,132}$ were discarded by a single consistency test, the data sets from Refs. ${ }^{104,113-115,117,129}$ were discarded by two tests and the the data sets from Refs. ${ }^{60,100,101,103,124,130}$ by three tests. Note that, for most data sets only a selection of tests could be applied due to missing properties that were not reported in these publications. For all but one VLE data set, the results of the three applicable tests reinforce each other. The only exception is the data set of Agrawal and Kofke: ${ }^{106}$ the data set was confirmed 
by the compressibility factor test and the Clausius-Clapeyron test, but discarded by the deviation test.

For many of the investigated data sets, the data points do not agree with the seven selected best data sets within the reported statistical uncertainties. Therefore, systematic simulation errors ${ }^{29}$ are hold responsible for these deviations.

Fitting the combined data of the seven best data sets (Refs. ${ }^{23,71,121,122,125-127,135,136}$ and the data set from the present work) with the correlations presented in Eqs. (6) - (9) yields a fit that is essentially the same as the one presented in Table 3. For all studied properties, the differences between both fits are well below the differences among the data from the seven data sets. Hence, we simply recommend using the correlations from Table 3. 


\section{Interfacial VLE data}

As for the bulk VLE properties, the computer experiment data for the surface tension were assessed with respect to their mutual agreement. To facilitate this assessment, a base correlation function was also used for the temperature dependence of the surface tension

$$
\gamma=A\left(1-\frac{T}{T_{c}}\right)^{b}
$$

where $A$ and $b$ are parameters. $T_{c}=1.321$ was specified for the critical temperature in Eq. (11). In an initial step, the surface tension data from Refs. ${ }^{138,142}$ were used for the parametrization of Eq. (11). The obtained values for the parameters are: $A=3.03327$ and $b=1.27748$. The absolute average deviation between correlation (11) and the data used for the parametrization is $1.2 \%$. It will be shown below, that Eq. (11) matches the best available guess for the surface tension within its estimated precision.

The surface tension data was also assessed with the deviation test: First, the data sets with the best mutual agreement were identified. From these data sets, a confidence interval $\pm X \%$ was estimated as measure for the precision that the surface tension is known. For the assessment, a data point was considered as an outlier, if its relative deviation to the base correlation (11) exceeds 2.5 times the number of $\pm X \%$. Again, data sets for which more than half of the data points violate the criterion were discarded. The remaining data sets were considered as confirmed.

Figure 6 shows the surface tension data as a function of the temperature. Overall, the agreement is significantly poorer than for the bulk VLE properties. The best mutual agreement is found for the data sets of Chen et al. ${ }^{24}$, Janeček et al. ${ }^{135}$, Janeček ${ }^{131}$, Werth et al. ${ }^{142}$, Werth et al. ${ }^{137}$, Lee et al. ${ }^{98}$, Martinez-Ruiz et al. ${ }^{134}$, Mecke et al. ${ }^{140}$, Nijmeijer et al. ${ }^{102}$, Stephan and Hasse ${ }^{138}$, Shen et al. ${ }^{141}$. These data sets agree approximately within $\pm 4 \%$ - excluding the region close to the critical point.

The following data sets agree well, i.e. are considered as confirmed, but exhibit a more pronounced scatter or systematic deviations from the afore mentioned data sets: Trokhymchuk and Alejandre ${ }^{114}$, Potoff and Panagiotopoulos ${ }^{116}$, Miyazaki et al. ${ }^{139}$, Galliero et al. ${ }^{132}$, Errington ${ }^{125}$, 
Baidakov et al., ${ }^{129}$ Baidakov et al. ${ }^{123}$. The data sets of Refs. ${ }^{99,105,111,112,115,117}$ were discarded since more than $50 \%$ of the respective data points exceed the confidence interval. Details are given in the supplementary material.

\section{Conclusions}

This work reviews and assesses thermophysical property data for the Lennard-Jones fluid. Literature data (approximately 35,000 data points) were digitalized, evaluated, and provided in the supplementary material as a consistent database. For the homogeneous state points, approximately $10 \%$ of the data were identified to be gross outliers by an EOS test. The lack of information on the statistical uncertainties in some studies is unfortunate.

Three independent consistency tests were employed for the evaluation of the VLE data: The compressibility factor test considers only the vapor pressure and the saturated vapor density, whereas the Clausius-Clapeyron test also considers the saturated liquid density and the enthalpy of vaporization. The third test evaluates the mutual agreement of data sets for each VLE property individually. The VLE data sets of Errington, ${ }^{126,127}$ Errington, ${ }^{125}$ Janeček et al., ${ }^{135,136}$ Lotfi et al., ${ }^{71}$ Mick et al., ${ }^{23}$ Okumura and Yonezawa,${ }^{121,122}$ and this work were confirmed by all tests and found to be the most precise. The precision with which the VLE of the LJ fluid is known is thereby estimated to be $\pm 1 \%$ for the vapor pressure, $\pm 0.2 \%$ for the saturated liquid density, $\pm 1 \%$ for the saturated vapor density, and $\pm 0.75 \%$ for the enthalpy of vaporization - excluding the region close to the critical point. Since almost every VLE data set - even the most precise contain individual outliers, we recommend to use the combination of the above listed data sets as

reference. Furthermore, the data sets of Hansen and Verlet, ${ }^{20}$ Martínez-Ruiz et al., ${ }^{134}$ Okumura and Yonezawa, ${ }^{118,119}$ Stephan and Hasse, ${ }^{138}$ Stoll et al., ${ }^{128}$ and Werth et al. ${ }^{137}$ were found to be accurate, but not as precise. The precision of the surface tension is estimated to be $\pm 4 \%$, i.e. significantly larger than the VLE bulk properties.

The EOS test presented here can be adopted easily to other applications in thermodynamics. A test based on the ideas described here could for example be used for testing VLE data of a given 
mixture based on models of the Gibbs excess energy $G^{\mathrm{E}}$.

In a follow-up work we will use the consolidated data base that is presented here to investigate the performance of equations of state for the Lennard-Jones fluid. Future work should focus on the region in the direct vicinity of the critical point, where the available VLE data and accordingly the location of the critical point agree less well.

Among the molecular model fluids, the Lennard-Jones fluid has a similar role as water has among real fluids: it is the fluid that has been studied most extensively and most thoroughly. The present work summarizes the present state of these studies. The accuracy with which the properties of the LJ fluid are known is assessed here. It can be assumed that it is a lower limit to the accuracy with which properties of fluids can presently be determined with molecular simulations. That accuracy often exceeds the statistical uncertainties that are reported for individual data points.

\section{Acknowledgement}

The authors gratefully acknowledge the pleasant correspondence and the making available of their numerical values by J. R. Errington, J. Janeček, E. A. Ustinov, J. Fischer, K. Meier, H. Okumura, U. Deiters, R.J. Sadus, R. J. Wheatly, J. Mairhofer, A. Köster, and P. Mausbach. The authors are thankful for the support of the digitalization by J. Staubach, M. Bubel, C. Balzer, and M. Urschel. The authors are thankful for funding of the present work in the frame of the TaLPas project and by the European Research Council (ERC) in the frame of the Advanced Grant ENRICO (grant agreement No. 694807). The simulations were carried out on the ELWE supercomputer at Regional University Computing Center Kaiserslautern (RHRK) under the grant TUK-TLMV. The

present research was conducted under the auspices of the Boltzmann-Zuse Society of Computational Molecular Engineering (BZS). 


\section{Appendix}

\section{Supporting Information}

The supplementary material contains the database as an .xls spreadsheet of all discussed thermophysical property data in this work: Each data point is listed with the reference of the original publication, temperature, density, the property itself, eventually the reported statistical uncertainty, and the result of the EOS test. All data are given in standard Lennard-Jones reduced units. In addition, detailed remarks on individual data sets report on conversion pitfalls, likely misprints, etc. The supplementary material also contains the results of all three VLE tests for the discarded VLE data sets. Also, details on the EOS test are given in the supplementary material. The supporting material is available free of charge via the Internet at [http://pubs.acs.org].

\section{Molecular simulations in this work}

\section{Homogeneous state point simulations}

Simulations in the homogeneous state were carried out in the NVT ensemble using Monte Carlo (MC) sampling with an acceptance ratio of 0.5. The simulations contained 1372 particles. The initial conditions were generated as a fcc lattice and subsequently equilibrated for $2.5 \cdot 10^{5}$ cycles and then sampled for $3 \cdot 10^{6}$ cycles, where one cycle corresponds to 1372 attempts for a translational move. The cut-off radius was half the edge length of a cubic simulation volume. Statistical uncertainties were estimated by the block averaging method of Flyvbjerg and Petersen. ${ }^{34}$ Numerical values of the simulation results are reported in the supplementary material. Simulations were carried out with the simulation program $m s 2 .{ }^{177}$

\section{VLE simulations}

The vapor-liquid equilibrium of the Lennard-Jones fluid was obtained with the Grand Equilibrium method of Vrabec and Hasse. ${ }^{179}$ Two simulations were subsequently carried out to obtain the VLE properties at a given temperature: First, the chemical potential and the molar volume was sampled in a $N p T$ MD liquid phase simulation. Then, the vapor phase was simulated in a 
pseudo- $\mu V T$ ensemble using a MC algorithm. The pressure of the liquid state, was chosen to be approximately $7 \%$ above the vapor pressure as estimated by the Lennard-Jones EOS from Ref. ${ }^{207}$ 2048 particles are initialized on a fcc lattice for the $N p T$ simulations and then equilibrated for $3 \cdot 10^{5}$ time steps. The time step was set to $\Delta \tau=0.001$. Velocity scaling was used to specify the temperature. The chemical potential was sampled with Widom's test particle method, ${ }^{176}$ inserting 2500 test particles every time step. The chemical potential and its pressure derivative, i.e. the molar volume, were then used during the second simulation to obtain the saturated vapor phase properties. The pseudo- $\mu V T$ simulation was carried out such that the specified chemical potential was not constant but a function of the actual pressure of the vapor phase. The vapor phase simulations were started with 1000 particles using MC sampling with an acceptance ratio of 0.5. The system was equilibrated for $10^{5}$ time steps and production was carried out for $5 \cdot 10^{5}$ time steps. The initial vapor density was also estimated with the Lennard-Jones EOS from Ref. ${ }^{207}$ As for the homogeneous state point simulations, the cut-off radius was half the edge length of a cubic simulation volume and the statistical uncertainty was estimated by the block averaging method. ${ }^{34}$ The vapor-liquid equilibrium was sampled at 31 temperatures between $T=0.69$ and 1.28 . Numerical values of the obtained vapor pressure, saturated densities, and enthalpy of vaporization are reported in the supplementary material. 


\section{References}

(1) Chirico, R. D.; Frenkel, M.; Magee, J. W.; Diky, V.; Muzny, C. D.; Kazakov, A. F.; Kroenlein, K.; Abdulagatov, I.; Hardin, G. R.; Acree, W. E.; Brenneke, J. F.; Brown, P. L.; Cummings, P. T.; de Loos, T. W.; Friend, D. G.; Goodwin, A. R. H.; Hansen, L. D.; Haynes, W. M.; Koga, N.; Mandelis, A.; Marsh, K. N.; Mathias, P. M.; McCabe, C.; OâĂŹConnell, J. P.; Pádua, A.; Rives, V.; Schick, C.; Trusler, J. P. M.; Vyazovkin, S.; Weir, R. D.;

Wu, J. Improvement of Quality in Publication of Experimental Thermophysical Property Data: Challenges, Assessment Tools, Global Implementation, and Online Support. J. Chem. Eng. Data 2013, 58, 2699-2716.

(2) Dong, Q.; Yan, X.; Wilhoit, R. C.; Hong, X.; Chirico, R. D.; Diky, V. V.; Frenkel, M. Data Quality Assurance for Thermophysical Property Databases: Applications to the TRC SOURCE Data System. J. Chem. Inf. Comp. Sci. 2002, 42, 473-480.

(3) Voigt, W.; Brendler, V.; Marsh, K.; Rarey, R.; Wanner, H.; Gaune-Escard, M.; Cloke, P.; Vercouter, T.; Bastrakov, E.; Hagemann, S. Quality Assurance in Thermodynamic Databases for Performance Assessment Studies in Waste Disposal. Pure Appl. Chem. 2007, 79.

(4) Kang, J. W.; Diky, V.; Chirico, R. D.; Magee, J. W.; Muzny, C. D.; Abdulagatov, I.; Kazakov, A. F.; Frenkel, M. Quality Assessment Algorithm for Vapor-Liquid Equilibrium Data. J. Chem. Eng. Data 2010, 55, 3631-3640.

(5) Kang, J. W.; Diky, V.; Chirico, R. D.; Magee, J. W.; Muzny, C. D.; Kazakov, A. F.; Kroenlein, K.; Frenkel, M. Algorithmic Framework for Quality Assessment of Phase Equilibrium Data. J. Chem. Eng. Data 2014, 59, 2283-2293.

(6) Dortmund Data Bank (accessed June 2019). 2019, www.ddbst.com.

(7) Linstrom, P.; Mallard, W. NIST Chemistry WebBook, NIST Standard Reference Database 
Number 69. website, National Institute of Standards and Technology, Gaithersburg MD (retrieved April 16, 2019).

(8) Jones, J. On the Determination of Molecular Fields. I. From the Variation of the Viscosity of a Gas with Temperature. Proc. R. Soc. Lon. A 1924, 106, 441-462.

(9) Jones, J. On the Determination of Molecular Fields. II. From the Equation of State of a Gas. Proc. R. Soc. Lon. A 1924, 106, 463-477.

(10) van Westen, T.; Gross, J. A Critical Evaluation of Perturbation Theories by Monte Carlo Simulation of the First Four Perturbation Terms in a Helmholtz Energy Expansion for the Lennard-Jones Fluid. J. Chem. Phys. 2017, 147, 014503.

(11) Lafitte, T.; Apostolakou, A.; Avendano, C.; Galindo, A.; Adjiman, C. S.; Müller, E. A.; Jackson, G. Accurate Statistical Associating Fluid Theory for Chain Molecules formed from Mie Segments. The Journal of Chemical Physics 2013, 139, 154504.

(12) Stephan, S.; Liu, J.; Langenbach, K.; Chapman, W. G.; Hasse, H. Vapor-Liquid Interface of the Lennard-Jones Truncated and Shifted Fluid: Comparison of Molecular Simulation, Density Gradient Theory, and Density Functional Theory. J. Phys. Chem. C 2018, 122, $24705-24715$.

(13) Metropolis, N.; Rosenbluth, A. W.; Rosenbluth, M. N.; Teller, A. H.; Teller, E. Equation of State Calculations by Fast Computing Machines. J. Chem. Phys. 1953, 21, 1087-1092.

(14) Rosenbluth, M. N.; Rosenbluth, A. W. Further Results on Monte Carlo Equations of State. J. Chem. Phys. 1954, 22, 881-884.

(15) Wood, W. W.; Parker, F. R. Monte Carlo Equation of State of Molecules Interacting with the Lennard-Jones Potential. I. A Supercritical Isotherm at about Twice the Critical Temperature. J. Chem. Phys. 1957, 27, 720-733.

(16) Alder, B. J.; Wainwright, T. E. Studies in Molecular Dynamics. I. General Method. J. Chem. Phys. 1959, 31, 459-466. 
(17) Rahman, A. Correlations in the Motion of Atoms in Liquid Argon. Phys. Rev. 1964, 136, A405-A411.

(18) Verlet, L. Computer Experiments on Classical Fluids. I. Thermodynamical Properties of Lennard-Jones Molecules. Phys. Rev. 1967, 159, 98-103.

(19) McDonald, I. R.; Singer, K. Machine Calculation of Thermodynamic Properties of a Simple Fluid at Supercritical Temperatures. J. Chem. Phys. 1967, 47, 4766-4772.

(20) Hansen, J.-P.; Verlet, L. Phase Transitions of the Lennard-Jones System. Phys. Rev. 1969, 184, 151-161.

(21) Stephan, S.; Horsch, M.; Vrabec, J.; Hasse, H. MolMod - an Open Access Database of Force Fields for Molecular Simulations of Fluids. Mol. Simulat. 2019, 45, 806-814.

(22) Rutkai, G.; Thol, M.; Span, R.; Vrabec, J. How Well Does the Lennard-Jones Potential Represent the Thermodynamic Properties of Noble Gases? Mol. Phys. 2017, 115, 11041121.

(23) Mick, J.; Hailat, E.; Russo, V.; Rushaidat, K.; Schwiebert, L.; Potoff, J. GPU-accelerated Gibbs Ensemble Monte Carlo Simulations of Lennard-Jonesium. Comput. Phys. Commun. 2013, 184, $2662-2669$.

(24) Chen, B.; Siepmann, J. I.; Klein, M. L. Direct Gibbs Ensemble Monte Carlo Simulations for Solid-Vapor Phase Equilibria: Applications to Lennard-Jonesium and Carbon Dioxide. J. Phys. Chem. B 2001, 105, 9840-9848.

(25) Chapela, G. A.; Scriven, L. E.; Davis, H. T. Molecular Dynamics for Discontinuous Potential. IV. Lennard-Jonesium. J. Chem. Phys. 1989, 91, 4307-4313.

(26) Koppenol, W.; Corish, J.; Garcia-Martinez, J.; Meija, J.; Reedijk, J. How to Name new Chemical Elements (IUPAC Recommendations 2016). Pure Appl. Chem. 2016, 88, 401405. 
(27) Allen, M. P.; Tildesley, D. J. Computer Simulation of Liquids; Oxford University Press: Oxford, 1989.

(28) Hasse, H.; Lenhard, J. In Mathematics as a Tool: Tracing New Roles of Mathematics in the Sciences; Lenhard, J., Carrier, M., Eds.; Springer International Publishing, 2017; pp 93-115.

(29) Schappals, M.; Mecklenfeld, A.; Kröger, L.; Botan, V.; Köster, A.; Stephan, S.; Garcia, E. J.; Rutkai, G.; Raabe, G.; Klein, P.; Leonhard, K.; Glass, C. W.; Lenhard, J.; Vrabec, J.; Hasse, H. Round Robin Study: Molecular Simulation of Thermodynamic Properties from Models with Internal Degrees of Freedom. J. Chem. Theory Comput. 2017, 13, 4270-4280.

(30) Frenkel, D. Simulations: The Dark Side. The European Physical Journal Plus 2013, 128, 10.

(31) Nezbeda, I.; Kolafa, J. The Use of Control Quantities in Computer Simulation Experiments: Application to the Exp-6 Potential Fluid. Mol. Simulat. 1995, 14, 153-163.

(32) Stephan, S.; Dyga, M.; Alhafez, I. A.; Urbassek, H. M.; Hasse, H. Statistical Uncertainties in Molecular Friction Simulations. in preparation 2019,

(33) Forte, E.; Jirasek, F.; Bortz, M.; Burger, J.; Vrabec, J.; Hasse, H. Digitalization in Thermodynamics. Chem. Ing. Tech. 2019, 91, 201-214.

(34) Flyvbjerg, H.; Petersen, H. G. Error Estimates on Averages of Correlated Data. J. Chem. Phys. 1989, 91, 461-466.

(35) Lyu, M. R., Ed. Handbood of Software Reliability Engineering; McGraw-Hill: New York, 1996.

(36) Kofke, D. A.; Cummings, P. T. Quantitative Comparison and Optimization of Methods for Evaluating the Chemical Potential by Molecular Simulation. Mol. Phys. 1997, 92, 973-996.

(37) Barnett, V.; Lewis, T. Outliers in Statistical Data; Wiley: Chichester, 1978. 
(38) Hodge, V.; Austin, J. A Survey of Outlier Detection Methodologies. Artif. Intell. Rev. 2004, 22, 85-126.

(39) Knorr, E. M.; Ng, R. T.; Tucakov, V. Distance-Based Outliers: Algorithms and Applications. The VLDB Journal 2000, 8, 237-253.

(40) Tong, H.; Crowe, C. M. Detection of Gross Erros in Data Reconciliation by Principal Component Analysis. AIChE J. 1995, 41, 1712-1722.

(41) Egan, W. J.; Morgan, S. L. Outlier Detection in Multivariate Analytical Chemical Data. Anal. Chem. 1998, 70, 2372-2379.

(42) Span, R.; Wagner, W. On the Extrapolation Behavior of Empirical Equations of State. Int. J. Thermophys. 1997, 18, 1415-1443.

(43) Deiters, U. K.; Neumaier, A. Computer Simulation of the Characteristic Curves of Pure Fluids. J. Chem. Eng. Data 2016, 61, 2720-2728.

(44) Fickett, W.; Wood, W. W. Shock Hugoniots for Liquid Argon. Phys. Fluids 1960, 3, 204209.

(45) McDonald, I. R.; Singer, K. Calculation of Thermodynamic Properties of Liquid Argon from Lennard-Jones Parameters by a Monte Carlo Method. Discuss. Faraday Soc. 1967, 43, 40-49.

(46) Verlet, L.; Levesque, D. On the Theory of Classical Fluids VI. Physica 1967, 36, 254-268.

(47) Wood, W. W. In Physics of Simple Liquids; Temperley, H. N. V., Rowlinson, J. S., Rushbrooke, G. S., Eds.; North Holland Publishing Company: Amsterdam, 1968; pp 115-230.

(48) Levesque, D.; Verlet, L. Perturbation Theory and Equation of State for Fluids. Phys. Rev. 1969, 182, 307-316.

(49) McDonald, I. R.; Singer, K. Examination of the Adequacy of the 12-6 Potential for Liquid Argon by Means of Monte Carlo Calculations. J. Chem. Phys. 1969, 50, 2308-2315. 
(50) Hansen, J.-P. Phase Transition of the Lennard-Jones System. II. High-Temperature Limit. Phys. Rev. A 1970, 2, 221-230.

(51) McDonald, I. R.; Woodcock, L. V. Triple-Dipole Dispersion Forces in Dense Fluids. J. Phys. C 1970, 3, 722 .

(52) Toxvaerd, S.; Praestgaard, E. Equation of State for a Lennard-Jones Fluid. J. Chem. Phys. 1970, 53, 2389-2392.

(53) Weeks, J. D.; Chandler, D.; Andersen, H. C. Role of Repulsive Forces in Determining the Equilibrium Structure of Simple Liquids. J. Chem. Phys. 1971, 54, 5237-5247.

(54) McDonald, I.; Singer, K. An Equation of State for Simple Liquids. Mol. Phys. 1972, 23, 29-40.

(55) Schofield, P. Computer Simulation Studies of the Liquid State. Comput. Phys. Commun. 1973, 5, 17-23.

(56) Streett, W. B.; Raveché, H. J.; Mountain, R. D. Monte Carlo Studies of the Fluid-Solid Phase Transition in the Lennard-Jones System. J. Chem. Phys. 1974, 61, 1960-1969.

(57) Adams, D. J. Grand Canonical Ensemble Monte Carlo for a Lennard-Jones Fluid. Mol. Phys. 1975, 29, 307-311.

(58) Adams, D. J. Calculating the Low Temperature Vapour Line by Monte Carlo. Mol. Phys. 1976, 32, 647-657.

(59) Carley, D. D. Integral Equation and Perturbation Method for Equations of State for a Low Temperature Lennard-Jones Gas. J. Chem. Phys. 1977, 67, 4812-4818.

(60) Adams, D. J. Calculating the High-Temperature Vapour Line by Monte Carlo. Mol. Phys. 1979, 37, 211-221.

(61) Nicolas, J. J.; Gubbins, K. E.; Streett, W. B.; Tildesley, D. J. Equation of State for the Lennard-Jones Fluid. Molecular Physics 1979, 37, 1429. 
(62) Ree, F. H. Analytic Representation of Thermodynamic Data for the Lennard-Jones Fluid. J. Chem. Phys. 1980, 73, 5401-5403.

(63) Yao, J.; Greenkorn, R.; Chao, K. Monte Carlo Simulation of the Grand Canonical Ensemble. Mol. Phys. 1982, 46, 587-594.

(64) Powles, J.; Evans, W.; Quirke, N. Non-Destructive Molecular-Dynamics Simulation of the Chemical Potential of a Fluid. Mol. Phys. 1982, 46, 1347-1370.

(65) Lucas, K. Angewandte Statistische Thermodynamik, 1st ed.; Springer Verlag: Berlin, 1986.

(66) Shaw, M. S. A Density of States Transformation Monte Carlo Method: Thermodynamics of the Lennard-Jones Fluid. J. Chem. Phys. 1988, 89, 2312-2323.

(67) Adachi, Y.; Fijihara, I.; Takamiya, M.; Nakanishi, K. Generalized Equation of State for Lennard-Jones Fluids - I. Pure Fluids and Simple Mixtures. Fluid Phase Equilibr. 1988, 39, $1-38$.

(68) Baranyai, A.; Evans, D. J. Direct Entropy Calculation from Computer Simulation of Liquids. Phys. Rev. A 1989, 40, 3817-3822.

(69) Saager, B.; Fischer, J. Predictive Power of Effective Intermolecular Pair Potentials: MD Simulation Results for Methane up to 1000 MPa. Fluid Phase Equilibr. 1990, 57, 35-46.

(70) Sowers, G. M.; Sandler, S. I. Equations of State from Generalized Perturbation Theory: Part II. The Lennard-Jones Fluid. Fluid Phase Equilibr. 1991, 67, 127-150.

(71) Lotfi, A.; Vrabec, J.; Fischer, J. Vapour Liquid Equilibria of the Lennard-Jones Fluid from the NpT Plus Test Particle Method. Mol. Phys. 1992, 76, 1319-1333.

(72) Giaquinta, P. V.; Giunta, G.; Prestipino Giarritta, S. Entropy and the Freezing of Simple Liquids. Phys. Rev. A 1992, 45, R6966-R6968.

(73) Johnson, J. K.; Zollweg, J. A.; Gubbins, K. E. The Lennard-Jones Equation of State Revisited. Mol. Phys. 1993, 78, 591. 
(74) Kolafa, J.; Vörtler, H. L.; Aim, K.; Nezbeda, I. The Lennard-Jones Fluid Revisited: Computer Simulation Results. Mol. Simulat. 1993, 11, 305-319.

(75) Miyano, Y. An Equation of State for Lennard-Jones Pure Fluids Applicable over a very wide Temperature Range. Fluid Phase Equilibr. 1993, 85, 71-80.

(76) Kolafa, J.; Nezbeda, I. The Lennard-Jones Fluid: an Accurate Analytic and TheoreticallyBased Equation of State. Fluid Phase Equilibr. 1994, 100, 1-34.

(77) Lustig, R. Statistical Thermodynamics in the Classical Molecular Dynamics Ensemble. III. Numerical Results. J. Chem. Phys. 1994, 100, 3068-3078.

(78) Mecke, M.; Müller, A.; Winkelmann, J.; Vrabec, J.; Fischer, J.; Span, R.; Wagner, W. An Accurate Van der Waals-Type Equation of State for the Lennard-Jones Fluid. Int. J. Thermophys. 1996, 17, 391-404.

(79) Roccatano, D.; Amadei, A.; Apol, M. E. F.; Di Nola, A.; Berendsen, H. J. C. Application of the Quasi-Gaussian Entropy Theory to Molecular Dynamics Simulations of Lennard-Jones Fluids. J. Chem. Phys. 1998, 109, 6358-6363.

(80) Meier, K. Computer Simulation and Interpretation of the Transport Coefficients of the Lennard-Jones Model Fluid. Dissertation, University of the Federal Armed Forces Hamburg, 2002.

(81) Linhart, A.; Chen, C.-C.; Vrabec, J.; Hasse, H. Thermal Properties of the Metastable Supersaturated Vapor of the Lennard-Jones Fluid. J. Chem. Phys. 2005, 122, 144506.

(82) Morsali, A.; Beyramabadi, S. A.; Bozorgmehr, M. R. Evaluation of P-V-T Differential Properties of the Lennard-Jones Fluid Using Radial Distribution Functions and Molecular Dynamics. Chem. Phys. 2007, 335, 194-200.

(83) Baidakov, V. G.; Protsenko, S. P.; Kozlova, Z. R. Thermal and Caloric Equations of State for Stable and Metastable Lennard-Jones Fluids: I. Molecular-Dynamics Simulations. Fluid Phase Equilibr. 2008, 263, 55-63. 
(84) Lustig, R. Direct Molecular NVT Simulation of the Isobaric Heat Capacity, Speed of Sound and Joule-Thomson Coefficient. Mol. Simulat. 2011, 37, 457-465.

(85) May, H.-O.; Mausbach, P. Fluid Properties from Equations of State Compared with Direct Molecular Simulations for the Lennard-Jones System. Aip Conf. Proc. 2012, 1501, 954-960.

(86) Yigzawe, T. M. Molecular Dynamics Simulation of the Thermodynamic Properties of Water and Atomistic Fluids. Dissertation, Swinburne University of Technology, Melbourne, 2012.

(87) Yigzawe, T. M.; Sadus, R. J. Intermolecular Interactions and the Thermodynamic Properties of Supercritical Fluids. J. Chem. Phys. 2013, 138, 194502.

(88) Mairhofer, J.; Sadus, R. J. Thermodynamic Properties of Supercritical n-m Lennard-Jones Fluids and Isochoric and Isobaric Heat Capacity Maxima and Minima. J. Chem. Phys. 2013, 139, 154503.

(89) private communication with Mairhofer, J. BASF, 2019. Data from Ref.: ${ }^{88}$, Thermodynamic Properties of Supercritical n-m Lennard-Jones Fluids and Isochoric and Isobaric Heat Capacity Maxima and Minima. J. Chem. Phys. 2013, 139, 154503.

(90) Thol, M.; Rutkai, G.; Köster, A.; Lustig, R.; Span, R.; Vrabec, J. Equation of State for the Lennard-Jones Fluid. J. Phys. Chem. Ref. Data 2016, 45, 023101.

(91) Köster, A.; Mausbach, P.; Vrabec, J. Premelting, Solid-Fluid Equilibria, and Thermodynamic Properties in the High Density Region Based on the Lennard-Jones Potential. $J$. Chem. Phys. 2017, 147, 144502.

(92) private communication with Köster, A. Universität Paderborn and Mausbach, P. Technical University of Cologne, 2019. Data from Ref.: ${ }^{91}$, Premelting, Solid-Fluid Equilibria, and Thermodynamic Properties in the High Density Region Based on the Lennard-Jones Potential. J. Chem. Phys. 2017, 147, 144502.

(93) private communication with Ustinov, E.A. Polytechnicheskaya St. Petersburg, 2019. Data from Ref.: ${ }^{94}$, Efficient Chemical Potential Evaluation with Kinetic Monte Carlo Method and 
Non-Uniform External Potential: Lennard-Jones Fluid, Liquid, and Solid. J. Chem. Phys. 2017, 147, 014105.

(94) Ustinov, E. A. Efficient Chemical Potential Evaluation with Kinetic Monte Carlo Method and Non-Uniform External Potential: Lennard-Jones Fluid, Liquid, and Solid. J. Chem. Phys. 2017, 147 , 014105.

(95) Schultz, A. J.; Kofke, D. A. Comprehensive High-Precision High-Accuracy Equation of State and Coexistence Properties for Classical Lennard-Jones Crystals and Low-Temperature Fluid Phases. J. Chem. Phys. 2018, 149, 204508.

(96) Torrie, G.; Valleau, J. Nonphysical Sampling Distributions in Monte Carlo Free-Energy Estimation: Umbrella Sampling. J. Comput. Phys. 1977, 23, 187-199.

(97) private communication with Deiters, U.K. University of Cologne, 2019. Data from Ref.: ${ }^{43}$, Computer Simulation of the Characteristic Curves of Pure Fluids. J. Chem. Eng. Data 2016, $61,2720-2728$.

(98) Lee, J. K.; Barker, J. A.; Pound, G. M. Surface Structure and Surface Tension: Perturbation Theory and Monte Carlo Calculation. J. Chem. Phys. 1974, 60, 1976-1980.

(99) Chapela, G. A.; Saville, G.; Thompson, S. M.; Rowlinson, J. S. Computer Simulation of a Gas-Liquid Surface. Part 1. J. Chem. Soc. Farad. T. 2. 1977, 73, 1133-1144.

(100) Panagiotopoulos, A. Z. Direct Determination of Phase Coexistence Properties of Fluids by Monte Carlo Simulation in a New Ensemble. Mol. Phys. 1987, 61, 813-826.

(101) Panagiotopoulos, A. Z.; Quirke, N.; Stapleton, M.; Tildesley, D. J. Phase Equilibria by Simulation in the Gibbs ensemble. Mol. Phys. 1988, 63, 527-545.

(102) Nijmeijer, M. J. P.; Bakker, A. F.; Bruin, C.; Sikkenk, J. H. A Molecular Dynamics Simulation of the Lennard-Jones Liquid-Vapor Interface. J. Chem. Phys. 1988, 89, 3789-3792. 
(103) Smit, B.; Frenkel, D. Calculation of the Chemical Potential in the Gibbs Ensemble. Mol. Phys. 1989, 68, 951-958.

(104) Kofke, D. A. Direct Evaluation of Phase Coexistence by Molecular Simulation via Integration along the Saturation Line. J. Chem. Phys. 1993, 98, 4149-4162.

(105) Holcomb, C. D.; Clancy, P.; Zollweg, J. A. A Critical Study of the Simulation of the LiquidVapour Interface of a Lennard-Jones Fluid. Mol. Phys. 1993, 78, 437-459.

(106) Agrawal, R.; Kofke, D. A. Thermodynamic and Structural Properties of Model Systems at Solid-Fluid Coexistence. Mol. Phys. 1995, 85, 43-59.

(107) Hunter, J. E.; Reinhardt, W. P. Finite-Size Scaling Behavior of the Free Energy Barrier Between Coexisting Phases: Determination of the Critical Temperature and Interfacial Tension of the Lennard-Jones Fluid. J. Chem. Phys. 1995, 103, 8627-8637.

(108) Sadus, R. J.; Prausnitz, J. M. Three-Body Interactions in Fluids from Molecular Simulation: Vapor-Liquid Phase Coexistence of Argon. J. Chem. Phys. 1996, 104, 4784-4787.

(109) Mecke, M.; Winkelmann, J.; Fischer, J. Molecular Dynamics Simulation of the Liquid-Vapor Interface: The Lennard-Jones Fluid. J. Chem. Phys. 1997, 107, 9264-9270.

(110) Plačkov, D.; Sadus, R. J. Molecular Simulation of Intermolecular Attraction and Repulsion in Coexisting Liquid and Vapour Phases. Fluid Phase Equilibr. 1997, 134, 77-85.

(111) Guo, M.; Peng, D.-Y.; C.-Y.Lu, B. On the Long-Range Corrections to Computer Simulation Results for the Lennard-Jones Vapor-Liquid Interface. Fluid Phase Equilibr. 1997, 130, 19 30.

(112) Guo, M.; Lu, B. C.-Y. Long Range Corrections to Thermodynamic Properties of Inhomogeneous Systems with Planar Interfaces. J. Chem. Phys. 1997, 106, 3688-3695.

(113) Martin, M. G.; Siepmann, J. I. Transferable Potentials for Phase Equilibria. 1. United-Atom Description of n-Alkanes. J. Phys. Chem. B 1998, 102, 2569-2577. 
(114) Trokhymchuk, A.; Alejandre, J. Computer Simulations of Liquid/Vapor Interface in Lennard-Jones Fluids: Some Questions and Answers. J. Chem. Phys. 1999, 111, 85108523.

(115) Anisimov, S. I.; Dunikov, D. O.; Zhakhovskii, V. V.; Malyshenko, S. P. Properties of a Liquid-Gas Interface at High-Rate Evaporation. J. Chem. Phys. 1999, 110, 8722-8729.

(116) Potoff, J. J.; Panagiotopoulos, A. Z. Surface Tension of the Three-Dimensional LennardJones Fluid from Histogram-Reweighting Monte Carlo Simulations. J. Chem. Phys. 2000, 112, 6411-6415.

(117) Baidakov, V.; Chernykh, G.; Protsenko, S. Effect of the Cut-Off Radius of the Intermolecular Potential on Phase Equilibrium and Surface Tension in Lennard-Jones Systems. Chem. Phys. Lett. 2000, 321, 315-320.

(118) private communication with Okumura, H. Keio University, 2019. Data from Ref.: ${ }^{119}$, LiquidVapor Coexistence Curves of Several Interatomic Model Potentials. J. Chem. Phys. 2000, $113,9162-9168$.

(119) Okumura, H.; Yonezawa, F. Liquid-Vapor Coexistence Curves of Several Interatomic Model Potentials. J. Chem. Phys. 2000, 113, 9162-9168.

(120) Shi, W.; Johnson, J. Histogram Reweighting and Finite-Size Scaling Study of the LennardJones Fluids. Fluid Phase Equilibria 2001, 187, 171-191.

(121) private communication with Okumura, H. Keio University, 2019. Data from Ref.: ${ }^{122}$, Reliable Determination of the Liquid-Vapor Critical Point by the NVT Plus Test Particle Method. J. Phys. Soc. Jpn. 2001, 70, 1990-1994.

(122) Okumura, H.; Yonezawa, F. Reliable Determination of the Liquid-Vapor Critical Point by the NVT Plus Test Particle Method. J. Phys. Soc. Jpn. 2001, 70, 1990-1994. 
(123) Baidakov, V. G.; Protsenko, S. P.; Chernykh, G. G.; Boltachev, G. S. Statistical Substantiation of the van der Waals Theory of Inhomogeneous Fluids. Phys. Rev. E 2002, 65, 041601.

(124) Kioupis, L. I.; Arya, G.; Maginn, E. J. Pressure-Enthalpy Driven Molecular Dynamics for Thermodynamic Property Calculation II: Applications. Fluid Phase Equilibr. 2002, 200, 93-110.

(125) Errington, J. R. Evaluating Surface Tension using Grand-Canonical Transition-Matrix Monte Carlo Simulation and Finite-Size Scaling. Phys. Rev. E 2003, 67, 012102.

(126) Errington, J. R. Direct Calculation of Liquid-Vapor Phase Equilibria from Transition Matrix Monte Carlo Simulation. J. Chem. Phys. 2003, 118, 9915-9925.

(127) private communication with Errington, J.R. University at Buffalo, 2019. Data from Ref.: ${ }^{126}$, Direct Calculation of Liquid-Vapor Phase Equilibria from Transition Matrix Monte Carlo Simulation. J. Chem. Phys. 2003, 118, 9915-9925.

(128) Stoll, J.; Vrabec, J.; Hasse, H. Comprehensive Study of the Vapour-Liquid Equilibria of the Pure Two-Centre Lennard-Jones Plus Pointdipole Fluid. Fluid Phase Equilibr. 2003, 209, $29-53$.

(129) Baidakov, V. G.; Protsenko, S. P.; Kozlova, Z. R.; Chernykh, G. G. Metastable Extension of the Liquid-Vapor Phase Equilibrium Curve and Surface Tension. J. Chem. Phys. 2007, 126, 214505.

(130) Betancourt-Cardenas, F.; Galicia-Luna, L.; Sandler, S. Equation of State for the LennardJones Fluid Based on the Perturbation Theory. Fluid Phase Equilibr. 2008, 264, 174-183.

(131) Janeček, J. Effect of the Interfacial Area on the Equilibrium Properties of Lennard-Jones Fluid. J. Chem. Phys. 2009, 131, 124513. 
(132) Galliero, G.; Pineiro, M. M.; Mendiboure, B.; Miqueu, C.; Lafitte, T.; Bessieres, D. Interfacial Properties of the Mie-6 Fluid: Molecular Simulations and Gradient Theory Results. J. Chem. Phys. 2009, 130, 104704.

(133) Sadus, R. J. Molecular Simulation of the Phase Behavior of Fluids and Fluid Mixtures using the Synthetic Method. J. Chem. Phys. 2012, 137, 054507.

(134) Martinez-Ruiz, F. J.; Blas, F. J.; Mendiboure, B.; Moreno-Ventas Bravo, A. I. Effect of Dispersive Long-Range Corrections to the Pressure Tensor: The Vapour-Liquid Interfacial Properties of the Lennard-Jones System Revisited. J. Chem. Phys. 2014, 141, 184701.

(135) Janeček, J.; Said-Aizpuru, O.; Paricaud, P. Long Range Corrections for Inhomogeneous Simulations of Mie n-m Potential. J. Chem. Theory Comput. 2017, 13, 4482-4491.

(136) Janeček, J.; Said-Aizpuru, O.; Paricaud, P. Long Range Corrections for Inhomogeneous Simulations of Mie n-m Potential. J. Chem. Theory Comput. 2017, 13, 4482-4491, (GEMC results from private communication with Janeček, 2019).

(137) Werth, S.; Stöbener, K.; Horsch, M.; Hasse, H. Simultaneous Description of Bulk and Interfacial Properties of Fluids by the Mie Potential. Mol. Phys. 2017, 115, 1017-1030.

(138) Stephan, S.; Hasse, H. Influence of Dispersive Long Range Interactions on Properties of Vapour-Liquid Interfaces of Binary Lennard-Jones Mixtures. submitted to Molecular Physics 2019 ,

(139) Miyazaki, J.; Barker, J. A.; Pound, G. M. A new Monte Carlo Method for Calculating Surface Tension. J. Chem. Phys. 1976, 64, 3364-3369.

(140) Mecke, M.; Müller, A.; Winkelmann, J.; Fischer, J. An Equation of State for Two-Center Lennard-Jones Fluids. Int. J. Thermophys. 1997, 18, 683-698.

(141) Shen, V. K.; Mountain, R. D.; Errington, J. R. Comparative Study of the Effect of Tail Corrections on Surface Tension Determined by Molecular Simulation. J. Phys. Chem. B 2007, 111, 6198-6207. 
(142) Werth, S.; Lishchuk, S. V.; Horsch, M.; Hasse, H. The Influence of the Liquid Slab Thickness on the Planar Vapor-Liquid Interfacial Tension. Physica A 2013, 392, 2359.

(143) van der Hoef, M. A. Free Energy of the Lennard-Jones Solid. J. Chem. Phys. 2000, 113, $8142-8148$.

(144) Barroso, M. A.; Ferreira, A. L. Solid-Fluid Coexistence of the Lennard-Jones System from Absolute Free Energy Calculations. J. Chem. Phys. 2002, 116, 7145-7150.

(145) Morris, J. R.; Song, X. The Melting Lines of Model Systems Calculated from Coexistence Simulations. J. Chem. Phys. 2002, 116, 9352-9358.

(146) Errington, J. R. Solid-Liquid Phase Coexistence of the Lennard-Jones System through Phase-Switch Monte Carlo Simulation. J. Chem. Phys. 2004, 120, 3130-3141.

(147) McNeil-Watson, G. C.; Wilding, N. B. Freezing Line of the Lennard-Jones Fluid: A Phase Switch Monte Carlo Study. J. Chem. Phys. 2006, 124, 064504.

(148) Mastny, E. A.; de Pablo, J. J. Melting Line of the Lennard-Jones System, Infinite Size, and Full Potential. J. Chem. Phys. 2007, 127, 104504.

(149) Ahmed, A.; Sadus, R. J. Solid-Liquid Equilibria and Triple Points of n-6 Lennard-Jones Fluids. J. Chem. Phys. 2009, 131, 174504.

(150) Ahmed, A.; Sadus, R. J. Erratum: Solid-Liquid Equilibria and Triple Points of n-6 LennardJones fluids [J. Chem. Phys. 131, 174504 (2009)]. J. Chem. Phys. 2010, 133, 229902.

(151) Sousa, J. M. G.; Ferreira, A. L.; Barroso, M. A. Determination of the Solid-Fluid Coexistence of the n-6 Lennard-Jones System from Free Energy Calculations. J. Chem. Phys. 2012, 136, 174502.

(152) private communication with Fischer, J. Universität fÂĺur Bodenkultur, 2019. Data from Ref.: ${ }^{153}$, Prediction of Gas PVT Data with Effective Intermolecular Potentials using the 
Haar-Shenker-Kohler Equation and Computer Simulations. Fluid Phase Equilibr. 1990, 54, $237-246$.

(153) Saager, B.; Lotfi, A.; Bohn, M.; Nguyen, V. N.; Fischer, J. Prediction of Gas PVT Data with Effective Intermolecular Potentials using the Haar-Shenker-Kohler Equation and Computer Simulations. Fluid Phase Equilibr. 1990, 54, 237 - 246.

(154) Boda, D.; Lukács, T.; Liszi, J.; Szalai, I. The Isochoric-, Isobaric- and Saturation-Heat Capacities of the Lennard-Jones Fluid from Equations of State and Monte Carlo Simulations. Fluid Phase Equilibr. 1996, 119, 1-16.

(155) private communication with Meier, K. Helmut-Schmidt-Universität, 2019. Data from Ref.: ${ }^{80}$, Computer Simulation and Interpretation of the Transport Coefficients of the Lennard-Jones Model Fluid. Dissertation, University of the Federal Armed Forces Hamburg, 2002.

(156) private communication with Mausbach, P. Technical University of Cologne, 2019. Additional unpublished data from Ref.: ${ }^{85}$, Fluid Properties from Equations of State Compared with Direct Molecular Simulations for the Lennard-Jones System. Aip Conf. Proc. 2012, 1501, $954-960$.

(157) Emampour, J. S.; Morsali, A.; Beyramabadi, S. A.; Bozorgmehr, M. R.; Khakzadan, K. The Pseudo Grüneisen Parameter in Dense Fluids from Distribution Functions. Int. J. Phys. Sci. 2011, 6, 5731-5737.

(158) Mausbach, P.; May, H.-O. Direct Molecular Simulation of the Grüneisen Parameter and Density Scaling Exponent in Fluid Systems. Fluid Phase Equilibr. 2014, 366, 108-116.

(159) Mausbach, P.; Köster, A.; Rutkai, G.; Thol, M.; Vrabec, J. Comparative Study of the Grüneisen Parameter for 28 Pure Fluids. J. Chem. Phys. 2016, 144, 244505.

(160) Weeks, J. D.; Chandler, D.; Andersen, H. C. Role of Repulsive Forces in Determining the Equilibrium Structure of Simple Liquids. J. Chem. Phys. 1971, 54, 5237-5247. 
(161) Han, K.-K. A new Monte Carlo Method for Estimating Free Energy and Chemical Potential. Phys. Lett. A 1992, 165, 28-32.

(162) Cuadros, F.; Mulero, A.; Ahumada, W. An Extensive Study of the Helmholtz Free Energy of Lennard-Jones Fluids using WCA Theory. Thermochim. Acta 1996, 277, 85-105.

(163) Hong, S. D.; Jhon, M. S. Calculation of Excess Free Energy from the Averaged Effective Acceptance Ratio for the Lennard-Jones Fluid and the Inverse Twelve Fluid. Chem. Phys. Lett. 1997, 273, 79-82.

(164) Hong, S.-D.; Jang, D.-J. Direct Calculation of the Free Energy Using Reduced Boltzmann Factors: Application to Lennard-Jones Fluids and Square-Well Fluids. B. Korean Chem. Soc. 2003, 24, 1351-1353.

(165) Bird, R. B.; Spotz, E. L.; Hirschfelder, J. O. The Third Virial Coefficient for Non-Polar Gases. J. Chem. Phys. 1950, 18, 1395-1402.

(166) Hirschfelder, J. O., Curtiss, C. F., Bird, R. B., Eds. Molecular Theory of Gases and Liquids; Wiley, New York, 1954.

(167) Barker, J. A.; Leonard, P. J.; Pompe, A. Fifth Virial Coefficients. J. Chem. Phys. 1966, 44, $4206-4211$.

(168) Sun, T.; Teja, A. S. An Equation of State for Real Fluids Based on the Lennard-Jones Potential. J. Phys. Chem. 1996, 100, 17365-17372.

(169) Shaul, K. R. S.; Schultz, A. J.; Kofke, D. A. The Effect of Truncation and Shift on Virial Coefficients of Lennard-Jones Potentials. Collect. Czech. Chem. C. 2010, 75, 447-462.

(170) Wheatley, R. J. Calculation of High-Order Virial Coefficients with Applications to Hard and Soft Spheres. Phys. Rev. Lett. 2013, 110, 200601.

(171) private communication with Wheatley, R.J. University of Nottingham, 2019. Data from 
Ref.: ${ }^{170}$, Calculation of High-Order Virial Coefficients with Applications to Hard and Soft Spheres. Phys. Rev. Lett. 2013, 110, 200601.

(172) Heyes, D. M.; Llaguno, C. T. Computer Simulation and Equation of State Study of the Boyle and Inversion Temperature of Simple Fluids. Chem. Phys. 1992, 168, 61-68.

(173) Colina, C. M.; Müller, E. A. Molecular Simulation of Joule-Thomson Inversion Curves. Int. J. Thermophys. 1999, 20, 229-235.

(174) Vrabec, J.; Kedia, G. K.; Hasse, H. Prediction of Joule-Thomson Inversion Curves for Pure Fluids and One Mixture by Molecular Simulation. Cryogenics 2005, 45, 253-258.

(175) Lustig, R. Statistical Analogues for Fundamental Equation of State Derivatives. Mol. Phys. 2012, 110, 3041-3052.

(176) Widom, B. Some Topics in the Theory of Fluids. J. Chem. Phys. 1963, 39, 2808.

(177) Rutkai, G.; Köster, A.; Guevara-Carrion, G.; Janzen, T.; Schappals, M.; Glass, C. W.; Bernreuther, M.; Wafai, A.; Stephan, S.; Kohns, M.; Reiser, S.; Deublein, S.; Horsch, M.; Hasse, H.; Vrabec, J. ms2: A Molecular Simulation Tool for Thermodynamic Properties, Release 3.0. Comput. Phys. Commun. 2017, 221, 343-351.

(178) Panagiotopoulos, A. Z. Monte Carlo Methods for Phase Equilibria of Fluids. J. Phys.: Condens. Matter 1999, 12, R25-R52.

(179) Vrabec, J.; Hasse, H. Grand Equilibrium: Vapour-Liquid Equilibria by a New Molecular Simulation Method. Mol. Phys. 2002, 100, 3375.

(180) Möller, D.; Fischer, J. Vapour Liquid Equilibrium of a Pure Fluid from Test Particle Method in Combination with NpT Molecular Dynamics Simulations. Mol. Phys. 1990, 69, 463-473.

(181) Kofke, D. A. Gibbs-Duhem Integration: A New Method for Direct Evaluation of Phase Coexistence by Molecular Simulation. Mol. Phys. 1993, 78, 1331-1336. 
(182) Ngale, K. N.; Desgranges, C.; Delhommelle, J. Wang-Landau Configurational Bias Monte Carlo Simulations: Vapour-Liquid Equilibria of Alkenes. Mol. Simulat. 2012, 38, 653-658.

(183) Ghoufi, A.; Malfreyt, P.; Tildesley, D. J. Computer Modelling of the Surface Tension of the Gas-Liquid and Liquid-Liquid Interface. Chem. Soc. Rev. 2016, 45, 1387-1409.

(184) Stephan, S.; Langenbach, K.; Hasse, H. Interfacial Properties of Binary Lennard-Jones Mixtures by Molecular Simulations and Density Gradient Theory. The Journal of Chemical Physics 2019, 150, 174704.

(185) Kataoka, Y.; Yamada, Y. Phase Diagram for a Lennard-Jones System Obtained through Constant-Pressure Molecular Dynamics Simulations. J. Comput. Chem., Jpn. 2014, 13, $257-262$.

(186) Kataoka, Y.; Yamada, Y. Thermodynamics and Molecular Dynamic Simulations of Threephase Equilibrium in Argon (v8). J. Comput. Chem., Jpn. 2014, 13, 130-138.

(187) Heng, V. R.; Nayhouse, M.; Crose, M.; Tran, A.; Orkoulas, G. Communication: Direct Determination of Triple-Point Coexistence through Cell Model Simulation. J. Chem. Phys. 2012, 137, 141101.

(188) Ladd, A.; Woodcock, L. Interfacial and Co-Existence Properties of the Lennard-Jones System at the Triple Point. Mol. Phys. 1978, 36, 611-619.

(189) Adams, D. J.; Adams, E. M.; Hills, G. J. The Computer Simulation of Polar Liquids. Mol. Phys. 1979, 38, 387-400.

(190) Amadei, A.; Apol, M. E. F.; Chillemi, G.; Berendsen, H. J. C.; Di Nola, A. Derivation of a General Fluid Equation of State Based on the Quasi-Gaussian Entropy Theory: Application to the Lennard-Jones Fluid. Mol. Phys. 1999, 96, 1469-1490.

(191) Caillol, J. M. Critical-Point of the Lennard-Jones Fluid: A Finite-Size Scaling Study. J. Chem. Phys. 1998, 109, 4885-4893. 
(192) Dunikov, D. O.; Malyshenko, S. P.; Zhakhovskii, V. V. Corresponding States Law and Molecular Dynamics Simulations of the Lennard-Jones Fluid. J. Chem. Phys. 2001, 115, 6623-6631.

(193) Hess, S. Augmented van der Waals Equation of State for the Lennard-Jones Fluid. Physica A 1999, 267, 58-70.

(194) Kim, S.; Henderson, D.; Barker, J. A. Perturbation Theory of Fluids and Deviations from Classical Behavior. Can. J. Phys. 1969, 47, 99-102.

(195) Koutras, N.; Harismiadis, V.; Tassios, D. A Simple Equation of State for the Lennard-Jones Fluid: A New Reference Term for Equations of State and Perturbation Theories. Fluid Phase Equilibr. 1992, '77, 13-38.

(196) May, H.-O.; Mausbach, P. Riemannian Geometry Study of Vapor-Liquid Phase Equilibria and Supercritical Behavior of the Lennard-Jones Fluid. Phys. Rev. E 2012, 85, 031201.

(197) Potoff, J. J.; Panagiotopoulos, A. Z. Critical Point and Phase Behavior of the Pure Fluid and a Lennard-Jones Mixture. J. Chem. Phys. 1998, 109, 10914-10920.

(198) Smit, B. Phase diagrams of Lennard-Jones fluids. J. Chem. Phys. 1992, 96, 8639-8640.

(199) Song, Y.; Mason, E. A. Statistical-mechanical theory of a new analytical equation of state. J. Chem. Phys. 1989, 91, 7840-7853.

(200) Sung, S. H.; Chandler, D. Optimized Cluster Theory, the Lennard-Jones Fluid, and the Liquid-Gas Phase Transition. Phys. Rev. A 1974, 9, 1688-1697.

(201) Kulinskii, V. L. The Critical Compressibility Factor of Fluids from the Global Isomorphism Approach. J. Chem. Phys. 2013, 139, 184119.

(202) Heyes, D. The Lennard-Jones Fluid in the Liquid-Vapour Critical Region. CMST 2015, 21, 169. 
(203) Heyes, D. M.; Woodcock, L. V. Critical and Supercritical Properties of Lennard-Jones Fluids. Fluid Phase Equilibr. 2013, 356, 301-308.

(204) Kataoka, Y. Extension of a van der Waals Equation of State to the Solid-liquid-vapor Phases of a Lennard-Jones System. J. Comput. Chem., Jpn. 2018, 4.

(205) Lishchuk, S. V.; Fischer, J. Communication: Long Range Corrections in Liquid-Vapor Interface Simulations. J. Chem. Phys. 2018, 149, 091102.

(206) Rousseeuw, P. J.; Croux, C. Alternatives to the Median Absolute Deviation. J. Am. Stat. Assoc. 1993, 88, 1273-1283.

(207) Stephan, S.; Staubach, J.; Forte, E.; Hasse, H. Equations of state of the Lennard-Jones fluid: a systematic comparison. in preparation $\mathbf{2 0 1 9}$,

(208) Mecke, M.; Müller, A.; Winkelmann, J.; Vrabec, J.; Fischer, J.; Span, R.; Wagner, W. Erratum - An Accurate Van der Waals-Type Equation of State for the Lennard-Jones Fluid. Int. J. Thermophys. 1998, 19, 1493-1498.

(209) Leys, C.; Ley, C.; Klein, O.; Bernard, P.; Licata, L. Detecting Outliers: Do Not Use Standard Deviation Around the Mean, Use Absolute Deviation Around the Median. J. Exp. Soc. Psychol. 2013, 49, 764-766.

(210) Gauss, C. Bestimmung der Genauigkeit der Beobachtungen. Zeitschrift für Astronomie und verwandte Wissenschaften 1816, 1, 185.

(211) Hampel, F. R. The Influence Curve and its Role in Robust Estimation. J. Am. Stat. Assoc. 1974, 69, 383-393.

(212) Huber, P. Robust Statistics; John Wiley: New York, 1981.

(213) Nezbeda, I. Simulations of Vapor-Liquid Equilibria: Routine versus Thoroughness. J. Chem. Eng. Data 2016, 61, 3964-3969. 
(214) Nezbeda, I. Vapour-Liquid Equilibria from Molecular Simulations: Some Issues Affecting Reliability and Reproducibility. Mol. Phys. 2019, 0, 1-8.

(215) Meroni, A.; Parola, A.; Reatto, L. Differential Approach to the Theory of Fluids. Phys. Rev. A 1990, 42, 6104-6115.

(216) private communication of Valleau with Mecke, M. Universität Halle-Wittenberg, 1995. Data from Ref.: ${ }^{78}$, Critical Point Data of the Lennard-Jones Fluid. 1994,

(217) Pérez-Pellitero, J.; Ungerer, P.; Orkoulas, G.; Mackie, A. D. Critical Point Estimation of the Lennard-Jones Pure Fluid and Binary Mixtures. J. Chem. Phys. 2006, 125, 054515. 
Table 2: Critical point data for the Lennard-Jones fluid obtained by computer experiment reported in the literature. The numbers in parentheses indicate the reported uncertainties of the last decimal digits.

\begin{tabular}{|c|c|c|c|c|}
\hline publication & year & $T_{c}$ & $\rho_{c}$ & $p_{c}$ \\
\hline Barker et al. ${ }^{167}$ & 1966 & 1.291 & 0.547 & 0.249 \\
\hline Verlet \& Levesque 46 & 1967 & 1.26 & 0.316 & 0.118 \\
\hline Verlet $^{18}$ & 1967 & 1.32 & 0.31 & 0.12276 \\
\hline \multirow{2}{*}{ Kim et al. ${ }^{194}$} & 1969 & 1.35 & 0.3 & 0.14 \\
\hline & & 1.28 & 0.29 & 0.13 \\
\hline Levesque \& Verlet $^{48}$ & 1969 & $1.36(3)$ & $0.36(3)$ & 0.151776 \\
\hline Sung \& Chandler 200 & 1974 & 1.348 & 0.349 & 0.148 \\
\hline Adams 60 & 1979 & $1.30(2)$ & $0.33(3)$ & $0.13(2)$ \\
\hline Panagiotopoulos ${ }^{100}$ & 1987 & $<1.35$ & $0.31(2)$ & \\
\hline Meroni et al. ${ }^{215}$ & 1990 & 1.3317 & 0.332 & \\
\hline Smit $^{198}$ & 1992 & $1.316(6)$ & $0.304(6)$ & \\
\hline Lotfi et al. ${ }^{71}$ & 1992 & 1.31 & 0.314 & \\
\hline \multirow[t]{2}{*}{ Kofke $e^{104}$} & 1993 & $1.321(4)$ & $0.306(1)$ & \\
\hline & & $1.324(12)$ & $0.305(2)$ & \\
\hline Valleau $^{78,216}$ & 1994 & $1.328(3)$ & & \\
\hline Hunter \& Reinhardt ${ }^{107}$ & 1995 & 1.32 & & \\
\hline Mecke et al. ${ }^{78}$ & 1996 & $1.328(3)$ & 0.3107 & \\
\hline Mecke et al. ${ }^{109}$ & 1997 & 1.32521 & & \\
\hline Caillol $^{191}$ & 1998 & $1.326(2)$ & $0.316(2)$ & $0.1472(3)$ \\
\hline \multirow[t]{2}{*}{ Martin $\&$ Siepmann ${ }^{113}$} & 1998 & $1.294(9)$ & $0.311(6)$ & 0.1144 \\
\hline & & $1.311(9)$ & & \\
\hline Potoff \& Panagiotopoulos ${ }^{197}$ & 1998 & $1.3120(7)$ & $0.316(1)$ & $0.1279(6)$ \\
\hline \multirow[t]{2}{*}{ Potoff \& Panagiotopoulos ${ }^{116}$} & 2000 & $1.311(2)$ & & \\
\hline & & $1.3120(7)$ & $0.316(2)$ & \\
\hline Okumura $\&$ Yonezawa ${ }^{119}$ & 2000 & $1.313(2)$ & $0.304(2)$ & $0.125(1)$ \\
\hline \multirow[t]{2}{*}{ Shi \& Johnson ${ }^{120}$} & 2001 & $1.3241(9)$ & $0.3165(7)$ & \\
\hline & & $1.3145(2)$ & $0.316(1)$ & \\
\hline Dunikov et al. ${ }^{192}$ & 2001 & $1.350(5)$ & $0.310(5)$ & $0.126(8)$ \\
\hline Okumura $\&$ Yonezawa ${ }^{122}$ & 2001 & $1.3207(4)$ & $0.316(1)$ & $0.1288(5)$ \\
\hline \multirow[t]{3}{*}{ Pérez-Pellitero et al. ${ }^{217}$} & 2006 & $1.3123(6)$ & $0.3174(6)$ & \\
\hline & & $1.3126(6)$ & $0.3174(6)$ & \\
\hline & & $1.313(1)$ & $0.317(1)$ & \\
\hline Betancourt-Cárdenas et al. ${ }^{130}$ & 2008 & $1.302(6)$ & $0.311(2)$ & $0.12(3)$ \\
\hline Kulinskii ${ }^{201}$ & 2013 & 1.333 & 0.325 & 0.122 \\
\hline Heyes \&6 Woodcock ${ }^{203}$ & 2013 & $1.3365(5)$ & & $0.1405(2)$ \\
\hline Heyes $^{202}$ & 2015 & $1.316(1)$ & $0.309(6)$ & \\
\hline Lishchuk \& Fischer ${ }^{205}$ & 2018 & 1.31766 & & \\
\hline
\end{tabular}


Table 3: Parameters of correlations (6) - (9) for the vapor pressure $p^{s}$, saturated liquid density $\rho^{\prime}$, saturated vapor density $\rho^{\prime \prime}$, and enthalpy of vaporization $\Delta h_{v}$.

\begin{tabular}{rrrrrrrrr}
\hline \hline & $p^{s}$ & Eq. (6) & \multicolumn{2}{c}{$\rho^{\prime}$} & Eq. (7) & $\rho^{\prime \prime}$ & Eq. (8) & \multicolumn{2}{c}{$\Delta h_{v}$ Eq. (9) } \\
\cline { 2 - 8 } & \multicolumn{1}{c}{$n_{i}$} & \multicolumn{1}{c}{$n_{i}$} & \multicolumn{1}{c}{$n_{i}$} & \multicolumn{1}{c}{$t_{i}$} & \multicolumn{1}{c}{$n_{i}$} & \multicolumn{1}{l}{$t_{i}$} \\
\hline 1 & 1.156551 & 1.341700 & 0.327140 & -8.135822 & 1.651685 & 6.456728 & 0.411342 \\
2 & -4.431519 & 2.075332 & 0.958759 & -102.919110 & 43.469214 & 2.700099 & 0.460416 \\
3 & -0.423028 & -2.123475 & 1.645654 & -3.037979 & 0.462877 & -3.073573 & 2.350953 \\
4 & 2.638743 & 0.328998 & 17.000001 & -44.381841 & 11.500462 & 3.149052 & 5.017010 \\
5 & & 1.386131 & 2.400858 & -34.55892948 & 5.394370 & & \\
\hline \hline
\end{tabular}



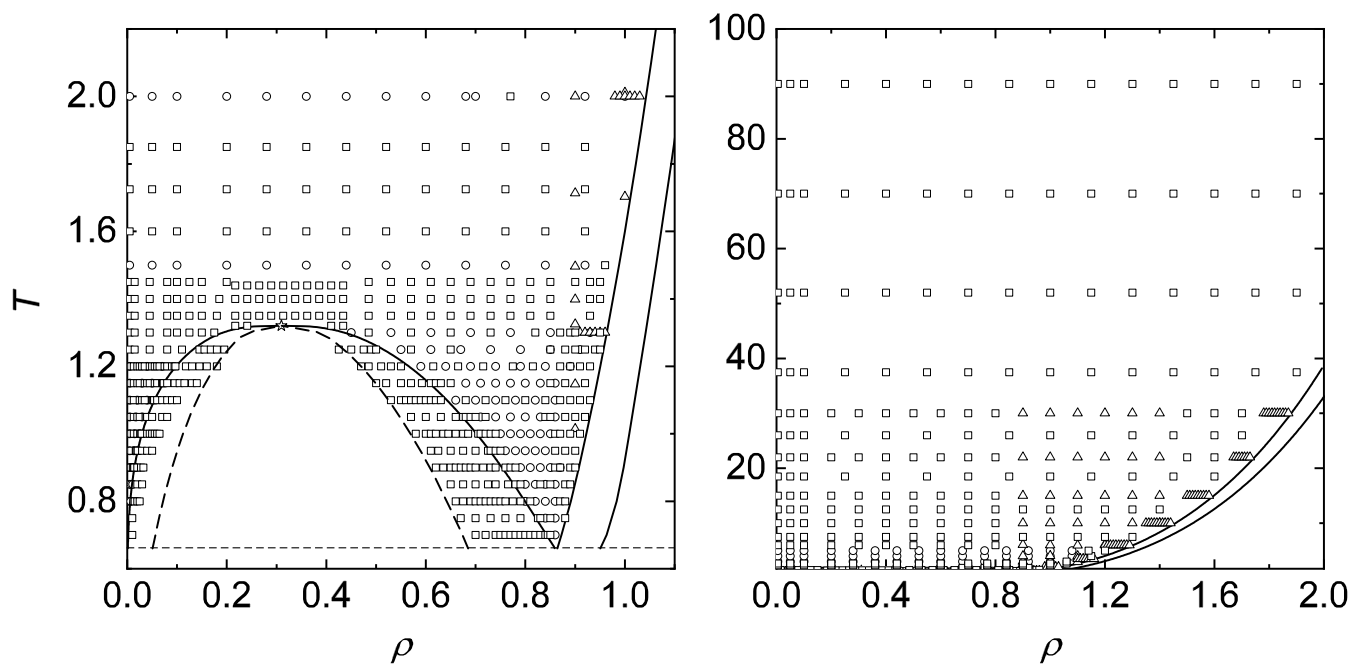

Figure 1: Overview of 963 state points that were studied with the Lustig formalism ${ }^{84,175}$ by different authors. Circles: Thol et al.; ${ }^{90}$ triangles: Köster et al.; ${ }^{91,92}$ squares: this work. Data for the Helmholtz energy and its density and inverse temperature derivatives up to second order are available for the stable state points. For the metastable state points, the derivatives are available up to first order. 


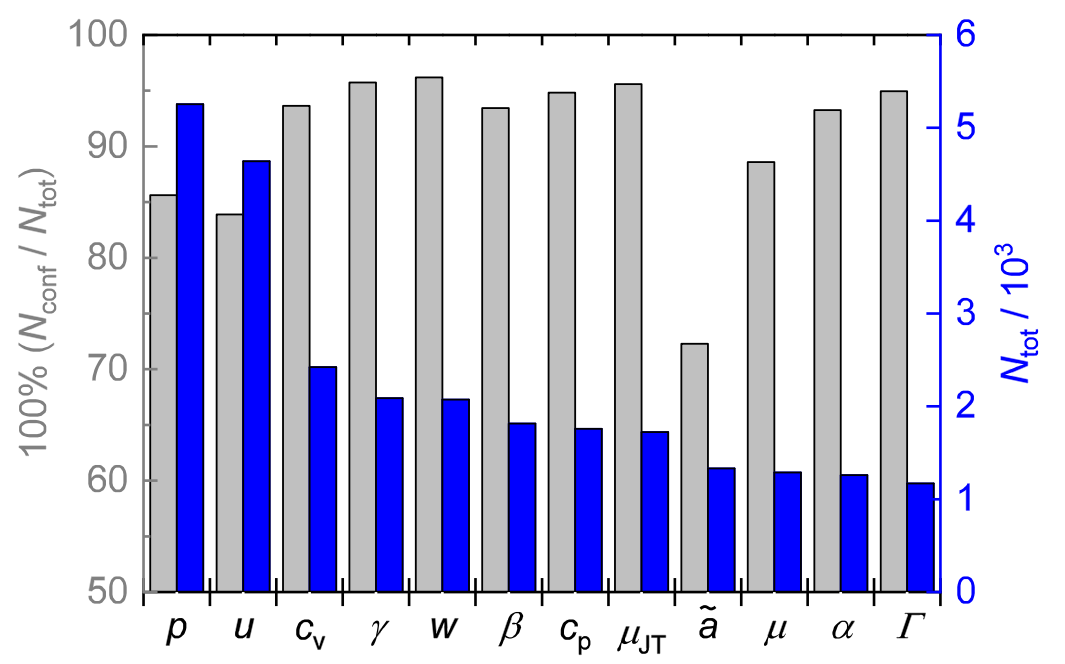

Figure 2: Statistics of the EOS test for homogeneous state points for different thermophysical properties: pressure $p$, internal energy $u$, isochoric heat capacity $c_{v}$, thermal pressure coefficient $\gamma$, speed of sound $w$, isothermal compressibility $\beta$, isobaric heat capacity $c_{p}$, Joule-Thomson coefficient $\mu_{\mathrm{JT}}$, Helmholtz energy divided by temperature $\tilde{a}=a / T$, chemical potential $\mu$, thermal expansion coefficient $\alpha$, and Grüneisen coefficient $\Gamma$. The left ordinate indicates the percentage of confirmed data and the right coordinate the total number of data points available for each thermophysical property. 


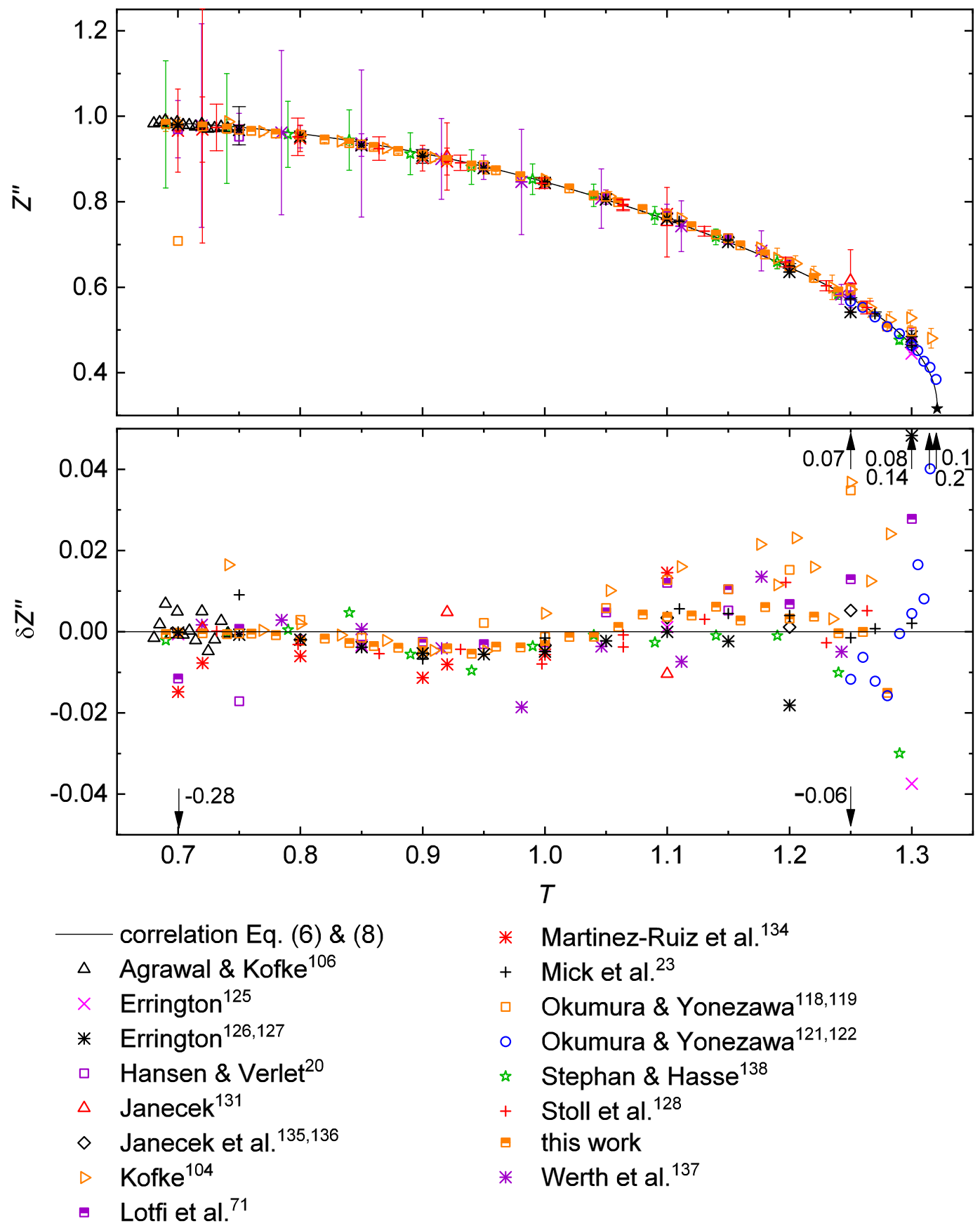

Figure 3: Compressibility factor test for the vapor-liquid equilibrium data of the Lennard-Jones fluid: saturated vapor phase compressibility factor $Z^{\prime \prime}$ as function of the temperature $T$ (top) and the relative deviation of $Z^{\prime \prime}$ from the base correlations (6) and (8) (bottom). The black filled star indicates the compressibility factor at the critical point according the base correlation. Error bars are omitted in the bottom plot to avoid visual clutter. 


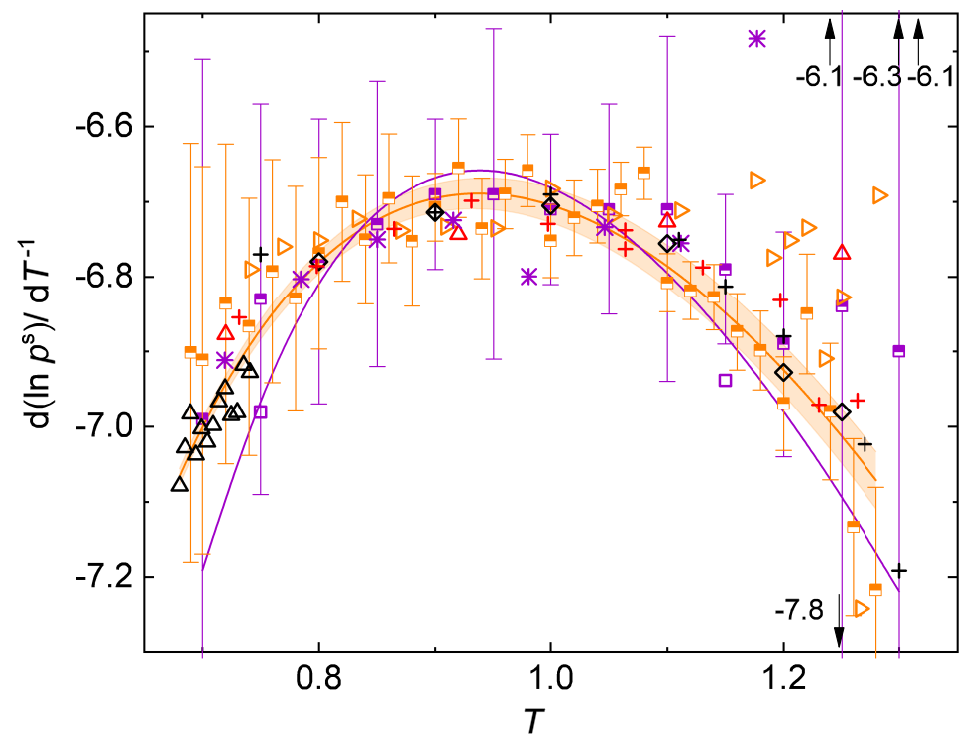

\begin{tabular}{|c|c|c|}
\hline & Eq. (6) & $\triangleright$ Kofke $^{104}$ \\
\hline & -Eq. (22) from Ref. ${ }^{12}$ & - Lotfi et al. ${ }^{71}$ \\
\hline$\Delta$ & Agrawal \& Kofke ${ }^{106}$ & + Mick et al. $^{23}$ \\
\hline 口 & Hansen \& Verlet ${ }^{20}$ & + Stoll et al. ${ }^{128}$ \\
\hline$\Delta$ & Janecek $^{131}$ & this work \\
\hline$\diamond$ & Janecek et al. 135,136 & * Werth et al. ${ }^{137}$ \\
\hline
\end{tabular}

Figure 4: Clausius-Clapeyron test according Eq. (10). Symbols indicate the RHS and the lines the LHS of Eq. (10). The orange shaded area indicates the statistical uncertainty of $\pm 2 \%$ computed for the LHS of Eq. (6). The purple line is Eq. (22) from Ref. ${ }^{71}$ 

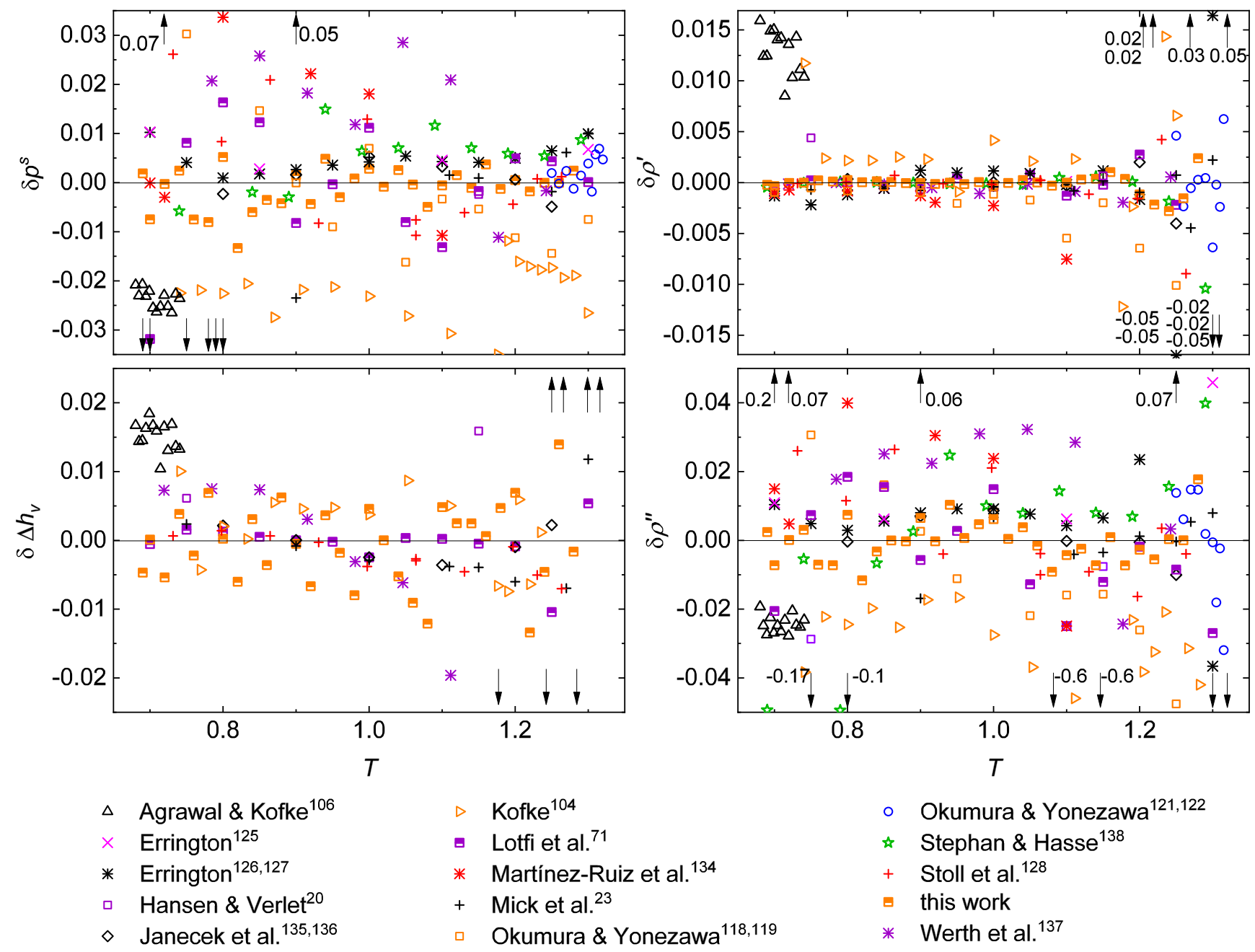

- Okumura \& Yonezawa ${ }^{121,122}$
* $\quad$ Stephan \& Hasse ${ }^{138}$
$+\quad$ Stoll et al. ${ }^{128}$
- this work $^{137}$
$* \quad$ Werth et al. ${ }^{137}$

Figure 5: Relative deviations of the vapor-liquid equilibrium data for the vapor pressure $p^{s}$, saturated liquid density $\rho^{\prime}$, saturated vapor density $\rho^{\prime \prime}$, and enthalpy of vaporization $\Delta h_{v}$ from correlations (6) - (9) as a function of the temperature $T$. Error bars were omitted to avoid visual clutter. For clarity, numerical values for out-of-range data points with negative deviation for the vapor pressure, the enthalpy of vaporization, and the saturated vapor density $\rho^{\prime \prime}$ are omitted in the respective plot; they lie in the range of $\delta p^{s}=-0.05 . .-0.16, \delta \Delta h_{v}=-0.15 . .0 .45$, and $\delta \rho^{\prime \prime}=-0.08 . .-0.2$. 

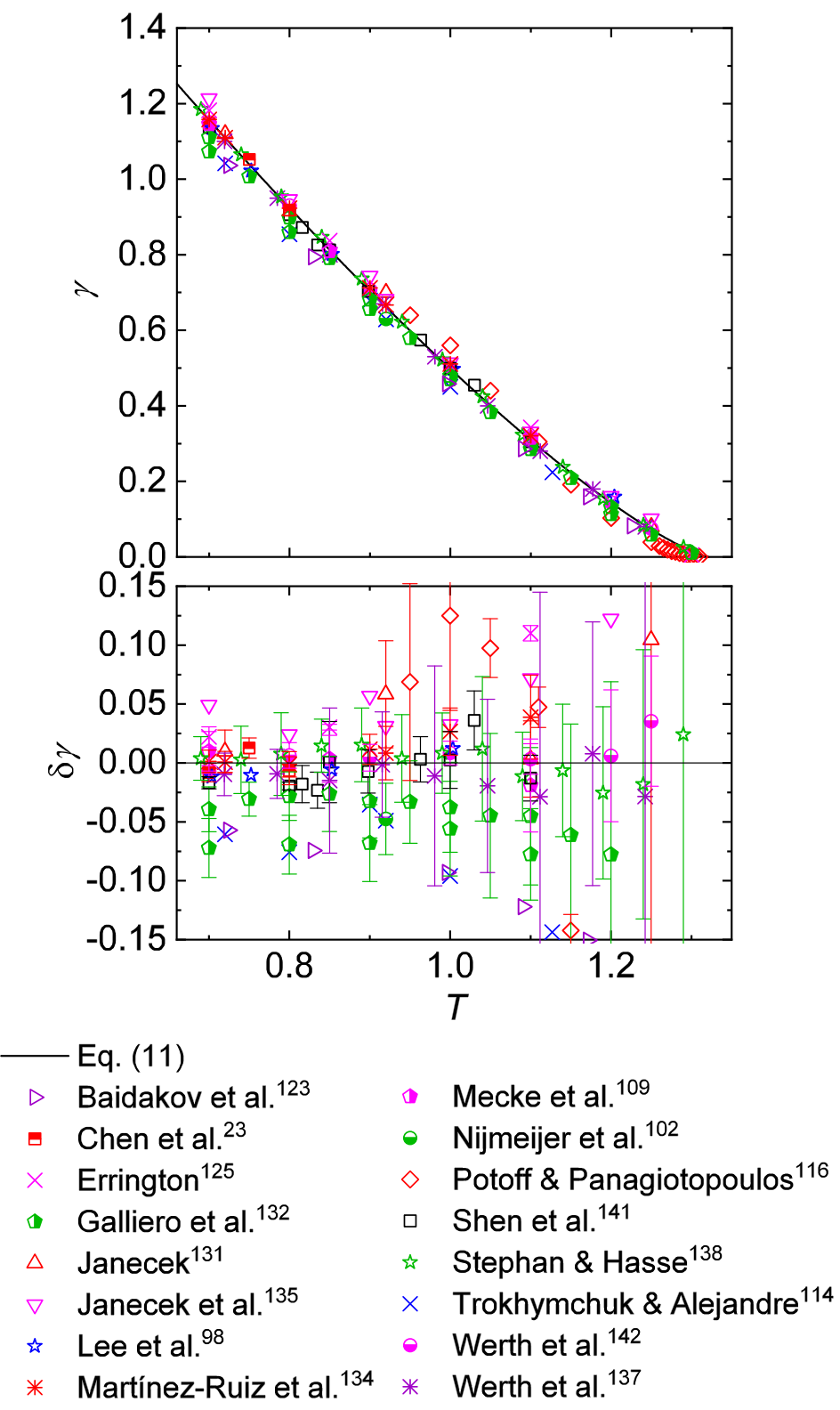

Figure 6: Surface tension of the LJ fluid as a function of the temperature (top) and the corresponding relative deviation plot (bottom). The black line (top) and baseline (bottom) indicates Eq. (11). Symbols indicate computer experiment data. For clarity, numerical values for out-of-range data points in the vicinity of the critical temperature are omitted in the deviation plot. 


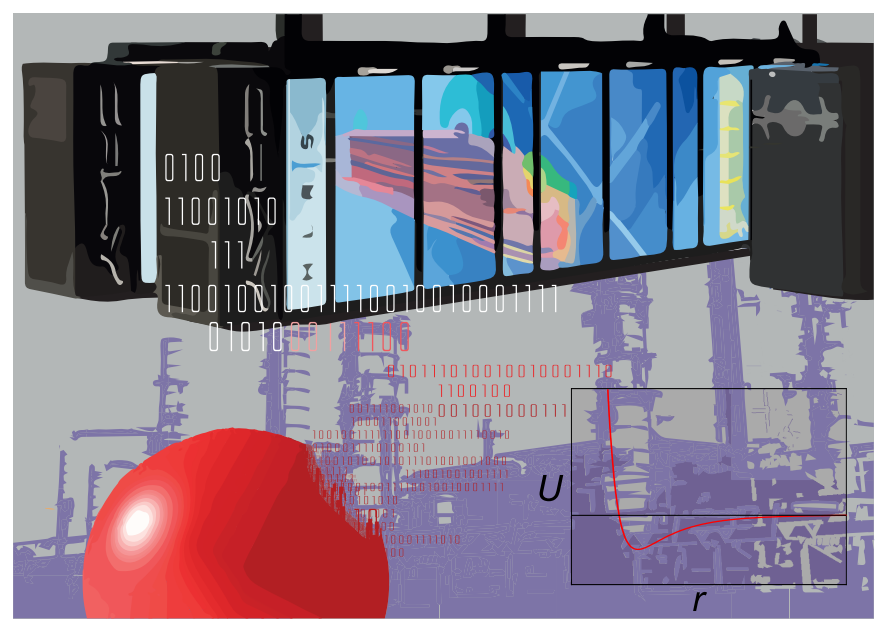

TOC graphic 


\section{Thermophysical Properties of the}

\section{Lennard-Jones Fluid: Database and}

\section{Data Assessment}

Simon Stephan ${ }^{1 *}$, Monika Thol $^{2}$, Jadran Vrabec ${ }^{3}$ and Hans Hasse ${ }^{1}$

${ }^{1}$ Laboratory of Engineering Thermodynamics (LTD), TU Kaiserslautern, Kaiserslautern 67663, Germany

${ }^{2}$ Lehrstuhl für Thermodynamik, Ruhr-Universität Bochum, Bochum 44801, Germany

${ }^{3}$ Thermodynamics and Process Engineering, TU Berlin, Berlin 10587, Germany 
This supplementary material of the publication Thermophysical Properties of the LennardJones Fluid: Database and Data Assessment contains the following:

1. The full database of thermophysical property data of the Lennard-Jones fluid discussed in this work is appended as an .xls-spreadsheet, including an indication for each bulk data point whether it was identified as an outlier by the EOS test.

2. Applied conversions for each property in a given publication included in the database to indicate how the values in the database were obtained from those printed in the publication. Also, remarks on individual publications are given regarding possible misprints, conversion pitfalls, outliers etc.

3. Additional information on the EOS test for the assessment of the homogeneous state points.

4. Details on the computation of thermophysical properties with the Lustig formalism.

5. Additional information on the compressibility factor test, the Clausius-Clapeyron test, and the deviation test of the VLE bulk data, cf. Fig. S2, S3, and S4, respectively. All available VLE data (both confirmed and discarded) are shown and discussed in detail.

6. Additional information on the surface tension data, cf. Fig. S5.

7. List of clear outliers (eight data point) from the seven best VLE data sets (Refs. ${ }^{23,71,121,122,125-127,135,136}$ and this work), cf. Table S4.

8. Numerical values of the VLE data set from this work, cf. Table S3. 


\section{Database: .xls-spreadsheet}

The entire database of thermophysical properties considered in this work is provided as an .xls spreadsheet in the electronic supplementary material.

\section{List of applied conversions and remarks}

For all considered studies in the database, the applied conversions from the numerical values printed in a publication to obtain the numerical values given in the database are given in Table S1. Data are consistently reported as residual properties (with respect to the ideal gas) and in the standard 'Lennard-Jones' units.

In Table S1, the hat ${ }^{\wedge}$ indicates the obtained value after the conversion as given in the database. Non-hat symbols were adopted as it stands in the corresponding publication. The asterisk indicates 'Lennard-Jones' units. Non-asterisk values indicate other units systems as applied in a given publication. The tilde $\tilde{a}$ indicates the Helmholtz energy per particle $a$ divided by the temperature, i.e. $\tilde{a}=\frac{a}{T}$. The $[$ Acronym $]$ indicates the string used in the electronic database to uniquely identify a certain publication. The state point is the given temperature and density and their respective conversions in case of homogeneous data; and only the temperature in case of VLE data. The column property lists the given thermophysical properties and their respective conversions of a given publication.

In cases where multiple results where reported for a given property and state point in a given publication (e.g. simulations performed with varying cut-off, particle number, sampling length etc.), only the numeric value with the largest particle number etc. were included in the database.

For the conversions given in Table $\mathrm{S} 1$, the constants $N_{\mathrm{AV}}=6.02214076 \cdot 10^{23} \mathrm{~mol}^{-1}$ and $k_{\mathrm{B}}=$ $1.380649 \cdot 10^{-23} \mathrm{~J} \cdot \mathrm{K}^{-1}$ were applied.

For the studies that report data based on the Lustig formalism, the Helmholtz energy and the respective derivatives are listed in Table S1. According the thermodynamic relations (see below), other properties were computed from that data and also included in the database. 
Table S1: List of conversions from the publications from the literature to the consistent units system (Lennard-Jones units \& residual properties with respect to the ideal gas).

\begin{tabular}{|c|c|c|}
\hline $\begin{array}{l}\text { Authors \& } \\
\text { [Acronym] }\end{array}$ & $\begin{array}{l}\text { state point } \\
T, \rho\end{array}$ & $\begin{array}{l}\text { thermophysical } \\
\text { properties }\end{array}$ \\
\hline Wood and Parker ${ }^{15}$ & $\widehat{T}^{*}=T^{*}$ & $\widehat{c}_{v}^{*}=\frac{3}{2}+C_{v}^{\prime} / R$ \\
\hline \multirow[t]{2}{*}{ [WOODPAR] } & $\widehat{\rho}^{*}=\left(\frac{v}{v^{*}}\right)^{-1}$ & $\widehat{u}_{\mathrm{res}}^{*}=\widehat{T}^{*} \cdot \frac{E^{\prime}}{R T}$ \\
\hline & & $\widehat{p}^{*}=\widehat{\rho}^{*} \cdot \widehat{T}^{*} \cdot \frac{p v}{R T}$ \\
\hline Hansen $^{50}$ & $\widehat{T}^{*}=T^{*}$ & $\widehat{p}^{*}=\widehat{\rho}^{*} \cdot \widehat{T}^{*} \cdot\left(\frac{\beta p}{\rho}\right)^{\mathrm{ex}}$ \\
\hline [HANSEN] & $\widehat{\rho}^{*}=\rho^{*}$ & $\widehat{u}_{\mathrm{res}}^{*}=\widehat{T}^{*} \cdot(\beta U)^{\mathrm{ex}}$ \\
\hline Levesque and Verlet ${ }^{48}$ & $\widehat{T}^{*}=T^{*}$ & $\widehat{\tilde{a}}_{\mathrm{res}, 00}^{*}=\frac{F_{1}}{N \cdot k_{\mathrm{B}} \cdot T}$ \\
\hline \multirow[t]{2}{*}{ [LEVEVER] } & $\widehat{\rho}^{*}=\rho^{*}$ & $\widehat{p}^{*}=\widehat{\rho}^{*} \cdot \widehat{T}^{*} \cdot Z$ \\
\hline & & $\widehat{u}_{\mathrm{res}}^{*}=u_{i}$ \\
\hline Thol et al. ${ }^{90}$ & $\widehat{T}^{*}=T^{*}$ & $\widehat{\tilde{a}}_{\mathrm{res}, 00}^{*}=A_{\mathrm{r}, 00}$ \\
\hline \multirow[t]{5}{*}[\mathrm{THOL}]{} & $\widehat{\rho}^{*}=\rho^{*}$ & $\widehat{\tilde{a}}_{\mathrm{res}, 01}^{*}=A_{\mathrm{r}, 01}$ \\
\hline & & $\widehat{\tilde{a}}_{\mathrm{res}, 10}^{*}=A_{\mathrm{r}, 10}$ \\
\hline & & $\widehat{\tilde{a}}_{\mathrm{res}, 11}^{*}=A_{\mathrm{r}, 11}$ \\
\hline & & $\widehat{\tilde{a}}_{\mathrm{res}, 02}^{*}=A_{\mathrm{r}, 02}$ \\
\hline & & $\widehat{\tilde{a}}_{\mathrm{res}, 20}^{*}=A_{\mathrm{r}, 20}$ \\
\hline
\end{tabular}

The numeric values provided in the supplementary material in Ref. ${ }^{90}$ contains some typos. Those are corrected here.

\begin{tabular}{lll}
\hline Verlet $^{18}$ & $\widehat{T}^{*}=T^{*}$ & $\widehat{p}^{*}=\widehat{\rho}^{*} \cdot \widehat{T}^{*} \cdot\left(\frac{\beta p}{\rho}\right)$ \\
[VERLET] & $\widehat{\rho}^{*}=\rho^{*}$ & $\widehat{u}_{\mathrm{res}}^{*}=U^{i}$ \\
\hline Hansen and Verlet ${ }^{20}$ & $\widehat{T}^{*}=T^{*}$ & $\widehat{p}^{*}=\widehat{\rho}^{*} \cdot \widehat{T}^{*} \cdot \frac{\beta p}{\rho}$ \\
[HANSVER] & $\widehat{\rho}^{*}=\rho^{*}$ & $\widehat{\tilde{a}}_{\mathrm{res}, 00}^{*}=\frac{F_{l}}{\widehat{T}^{*}}-\ln \left(\widehat{\rho}^{*}\right)+1$ \\
& & $\widehat{p}_{v}^{*}=p_{v}^{*}$ \\
& & $\Delta \widehat{h}_{v}^{*}=\mathcal{L}$ \\
\hline McDonald and Singer & & $\widehat{p}^{*}=p^{*}$ \\
\hline \hline
\end{tabular}

Continued on next page 


\begin{tabular}{|c|c|c|}
\hline $\begin{array}{l}\text { Authors \& } \\
{[\text { Acronym] }}\end{array}$ & $\begin{array}{l}\text { state point } \\
T, \rho\end{array}$ & $\begin{array}{l}\text { thermophysical } \\
\text { properties }\end{array}$ \\
\hline$[\mathrm{MCDSING}]$ & $\widehat{\rho}^{*}=\rho^{*}$ & $\widehat{u}_{\mathrm{res}}^{*}=u_{r}^{*}$ \\
\hline Weeks et al. ${ }^{160}$ & $\widehat{T}^{*}=T^{*}$ & $\widehat{\tilde{a}}_{\mathrm{res}, 00}^{*}=-\frac{\beta \Delta A}{N}$ \\
\hline [WEEKCHA1] & $\widehat{\rho}^{*}=\rho^{*}$ & \\
\hline Adams $^{57}$ & $\widehat{T}^{*}=T^{*}$ & $\widehat{\alpha}^{*}=\alpha^{*}$ \\
\hline \multirow[t]{6}{*}[\mathrm{ADAMS}]{} & $\widehat{\rho}^{*}=\rho^{*}$ & $\widehat{\beta}^{*}=\beta^{*}$ \\
\hline & & $\widehat{\gamma}^{*}=\gamma^{*}$ \\
\hline & & $\widehat{p}^{*}=p *$ \\
\hline & & $\widehat{\mu}_{\mathrm{res}}^{*}=\mu_{r}^{*}$ \\
\hline & & $\widehat{u}_{\mathrm{res}}^{*}=-u_{r}^{*}$ \\
\hline & & $\widehat{c}_{v}^{*}=\frac{3}{2}+c_{v, r}^{*}$ \\
\hline$R e e^{62}$ & $\widehat{T}^{*}=T^{*}$ & $\widehat{p}^{*}=\widehat{\rho}^{*} \cdot \widehat{T}^{*} \cdot \frac{P}{\rho k T}$ \\
\hline$[\mathrm{REE}]$ & $\widehat{\rho}^{*}=\rho^{*}$ & $\widehat{u}_{\mathrm{res}}^{*}=\frac{U^{e}}{k T} \cdot \widehat{T}^{*}$ \\
\hline Panagiotopoulos ${ }^{100}$ & $\widehat{T}^{*}=T^{*}$ & $\widehat{p}_{v}^{*}=P^{*}$ \\
\hline$[\mathrm{PANAGIO}]$ & $\widehat{\rho}^{*}=\rho^{*}$ & $\Delta \widehat{h}_{v}^{*}=\left(E_{\mathrm{v}}^{*}+\frac{\widehat{p}_{\mathrm{v}}^{*}}{\widehat{\rho}_{\mathrm{v}}^{*}}-\left(E_{1}^{*}+\frac{\widehat{p}_{1}^{*}}{\widehat{\rho}_{1}^{*}}\right)\right)$ \\
\hline Shaw $^{66}$ & $\widehat{T}^{*}=T^{*}$ & $\widehat{p}^{*}=p^{*}$ \\
\hline$[\mathrm{SHAW}]$ & $\widehat{\rho}^{*}=\rho^{*}$ & $\widehat{u}_{\mathrm{res}}^{*}=u_{r}^{*}$ \\
\hline Panagiotopoulos et al. ${ }^{101}$ & $\widehat{T}^{*}=T^{*}$ & $\widehat{p}_{v}^{*}=P$ \\
\hline$[\mathrm{PANQUIR}]$ & $\widehat{\rho}^{*}=\rho^{*}$ & $\Delta \widehat{h}_{v}^{*}=\left(E^{\mathrm{G}}+\frac{p^{\mathrm{G}}}{\rho^{\mathrm{G}}}-\left(E^{\mathrm{L}}+\frac{p^{\mathrm{L}}}{\rho^{\mathrm{L}}}\right)\right)$ \\
\hline Smit and Frenkel ${ }^{103}$ & $\widehat{T}^{*}=T^{*}$ & $\Delta \widehat{h}_{v}^{*}=\left(E_{\mathrm{v}}^{*}+\frac{\widehat{P}_{\mathrm{v}}^{*}}{\widehat{\rho}_{\mathrm{v}}^{*}}-\left(E_{\mathrm{l}}^{*}+\frac{\widehat{p}_{1}^{*}}{\widehat{\rho}_{1}^{*}}\right)\right)$ \\
\hline [SMITFRE] & $\widehat{\rho}^{*}=\rho^{*}$ & $\widehat{p}_{v}^{*}=p_{v}^{*}$ \\
\hline Meier ${ }^{80,155}$ & $\widehat{T}^{*}=T^{*}$ & $\widehat{c}_{v}^{*}=c_{v}^{*}$ \\
\hline \multirow[t]{4}{*}{ [MEIER] } & $\widehat{\rho}^{*}=\rho^{*}$ & $\widehat{p}^{*}=p^{*}$ \\
\hline & & $\widehat{u}_{\mathrm{res}}^{*}=u_{\mathrm{r}}^{*}$ \\
\hline & & $\widehat{w}^{*}=w_{o}^{\prime *}$ \\
\hline & & $\widehat{\gamma}^{*}=\gamma^{*}$ \\
\hline
\end{tabular}

Continued on next page 


\begin{tabular}{|c|c|c|}
\hline $\begin{array}{l}\text { Authors \& } \\
\text { [Acronym] }\end{array}$ & $\begin{array}{l}\text { state point } \\
T, \rho\end{array}$ & $\begin{array}{l}\text { thermophysical } \\
\text { properties }\end{array}$ \\
\hline \multicolumn{3}{|c|}{$\begin{array}{l}\text { Two typos in Ref. }{ }^{80} \text { are known }{ }^{155} \text { that were corrected in the database: A '0' is miss- } \\
\text { ing for the numbers of the reported pressure of two state points }\left(\widehat{\rho}^{*}=0.01 \& \widehat{T}^{*}=\right. \\
\left.0.900501 \text { and } \widehat{\rho}^{*}=0.005 \& \widehat{T}^{*}=0.900929\right) \text {. }\end{array}$} \\
\hline Johnson et al. ${ }^{73}$ & $\widehat{T}^{*}=T^{*}$ & $\widehat{p}^{*}=p^{*}$ \\
\hline [JOHNSON] & $\widehat{\rho}^{*}=\rho^{*}$ & $\widehat{u}_{\mathrm{res}}^{*}=u_{r}^{*}$ \\
\hline Hunter and Reinhardt ${ }^{107}$ & $\widehat{T}^{*}=T^{*}$ & $\widehat{p}_{v}^{*}=p_{v}^{*}$ \\
\hline [HUNTERR] & $\widehat{\rho}^{*}=\rho^{*}$ & \\
\hline McDonald and Singer ${ }^{45}$ & $\widehat{T}^{*}=\frac{T \cdot k_{\mathrm{B}}}{\varepsilon}$ & $\widehat{p}^{*}=\frac{10^{-23} \cdot \sigma^{3} \cdot p^{*}}{\varepsilon}$ \\
\hline \multirow[t]{6}{*}{ [MCDSIN1] } & $\widehat{\rho}^{*}=\frac{10^{-24} \cdot \sigma^{3} \cdot N_{\mathrm{AV}}}{v}$ & $\widehat{u}_{\mathrm{res}}^{*}=-u_{\mathrm{r}}^{*} \cdot \widehat{T}^{*}$ \\
\hline & & $\widehat{\alpha}^{*}=\frac{\alpha^{*} \cdot 10^{-3} \cdot \varepsilon}{k_{\mathrm{B}}}$ \\
\hline & & $\widehat{\beta}^{*}=\frac{\beta^{*} \cdot 10^{21} \cdot \varepsilon}{\sigma^{3}}$ \\
\hline & & $\widehat{\gamma}^{*}=\frac{\gamma^{*} \cdot 10^{-24} \cdot \sigma^{3}}{k_{\mathrm{B}}}$ \\
\hline & & $\widehat{c}_{v}^{*}=\frac{3}{2}+c_{v, \mathrm{r}}^{*}$ \\
\hline & & with $\frac{\varepsilon}{k_{\mathrm{B}}}=119.76 \mathrm{~K}, \sigma=3.405 \AA$ \\
\hline McDonald and Singer ${ }^{19}$ & $\widehat{T}^{*}=\frac{k_{\mathrm{B}}(T+273.15 \mathrm{~K})}{\varepsilon}$ & $\widehat{p}^{*}=Z \cdot \widehat{\rho}^{*} \cdot \widehat{T}^{*}$ \\
\hline \multirow[t]{3}{*}{ [MCDSIN2] } & $\widehat{\rho}^{*}=\left(\frac{v}{v^{*}}\right)^{-1}$ & $\widehat{u}_{\mathrm{res}}^{*}=-\widehat{T}^{*} \cdot u_{\mathrm{r}}^{*}$ \\
\hline & & $\widehat{c}_{v}^{*}=\frac{3}{2}+c_{v, \mathrm{r}}^{*}$ \\
\hline & & with $\frac{\varepsilon}{\mathrm{k}_{\mathrm{B}}}=119.76 \mathrm{~K}, \sigma=3.405 \AA$ \\
\hline Barker et al. ${ }^{167}$ & $\widehat{T}^{*}=T^{*}$ & $\widehat{B}=\frac{2}{3} \pi \cdot B^{*}$ \\
\hline [BARLEOP] & & $\widehat{C}=\left(\frac{2}{3} \pi\right)^{2} \cdot C^{*}$ \\
\hline McDonald and Singer ${ }^{49}$ & $\widehat{T}^{*}=T \frac{k_{\mathrm{B}}}{\varepsilon}$ & $\widehat{p}^{*}=\frac{P \cdot 101.325 \cdot \sigma^{3} \cdot 10^{-27}}{\varepsilon}$ \\
\hline [MCDSIN3] & $\widehat{\rho}^{*}=\frac{10^{-24} \cdot \sigma^{3} \cdot N_{\mathrm{AV}}}{V}$ & $\widehat{u}_{\mathrm{res}}^{*}=\frac{-u \cdot 4.184}{N_{\mathrm{AV}} \cdot \varepsilon}$ \\
\hline & & with $\frac{\varepsilon}{k_{\mathrm{B}}}=119.8 \mathrm{~K}, \sigma=3.405 \AA$ \\
\hline Adams $^{60}$ & $\widehat{T}^{*}=T^{*}$ & $\widehat{c}_{v}^{*}=\frac{3}{2}+c_{v, \mathrm{r}}^{*}$ \\
\hline [ADAMS2] & $\widehat{\rho}^{*}=\rho^{*}$ & $\widehat{\mu}^{*}=\widetilde{\mu}^{\prime}$ \\
\hline
\end{tabular}

Continued on next page 


\begin{tabular}{|c|c|c|}
\hline $\begin{array}{l}\text { Authors \& } \\
\text { [Acronym] }\end{array}$ & $\begin{array}{l}\text { state point } \\
T, \rho\end{array}$ & $\begin{array}{l}\text { thermophysical } \\
\text { properties }\end{array}$ \\
\hline & & $\widehat{p}^{*}=p^{*}$ \\
\hline & & $\widehat{u}_{\mathrm{res}}^{*}=u_{\mathrm{r}}^{*}$ \\
\hline & & $\widehat{\beta}^{*}=\widetilde{\beta}$ \\
\hline & & $\Delta \widehat{h}_{v}^{*}=\Delta \widetilde{h}$ \\
\hline Sowers and Sandler ${ }^{70}$ & $\widehat{T}^{*}=T^{*}$ & $\widehat{p}^{*}=Z \cdot \widehat{\rho}^{*} \cdot \widehat{T}^{*}$ \\
\hline [SOWSAND] & $\widehat{\rho}^{*}=\rho^{*}$ & $\widehat{u}_{\mathrm{res}}^{*}=u_{\mathrm{r}}^{*}$ \\
\hline Shi and Johnson ${ }^{120}$ & $\widehat{T}^{*}=T^{*}$ & \\
\hline [SHIJOHN] & $\widehat{\rho}^{*}=\rho^{*}$ & \\
\hline Morsali et al. ${ }^{82}$ & $\widehat{T}^{*}=T^{*}$ & $\widehat{\beta}^{*}=\kappa_{\mathrm{MD}}^{*}$ \\
\hline \multirow[t]{2}{*}[\text{MORSALI}]{} & $\widehat{\rho}^{*}=\rho^{*}$ & $\widehat{\gamma}^{*}=\gamma_{\mathrm{MD}}^{*}$ \\
\hline & & $\widehat{p}^{*}=p^{*}$ \\
\hline Kolafa and Nezbeda ${ }^{76}$ & $\widehat{T}^{*}=T^{*}$ & $\widehat{p}^{*}=p^{*}$ \\
\hline [KOLNEZB] & $\widehat{\rho}^{*}=\rho^{*}$ & $\widehat{u}_{\mathrm{res}}^{*}=u_{\mathrm{r}}^{*}$ \\
\hline Lotfi et al. ${ }^{71}$ & $\widehat{T}^{*}=T^{*}$ & $\widehat{\beta}^{*}=\beta *$ \\
\hline \multirow[t]{4}{*}{ [LOTVRAB] } & $\widehat{\rho}^{*}=\rho^{*}$ & $\widehat{p}^{*}=p^{*}$ \\
\hline & & $\widehat{u}_{\mathrm{res}}^{*}=u_{r}^{*}$ \\
\hline & & $\widehat{\Delta h}_{v}^{*}=h^{\prime \prime}-h^{\prime *}$ \\
\hline & & $\widehat{\mu}_{\mathrm{res}}^{*}=\left(\widetilde{\mu}-\ln \left(\widehat{\rho}^{*}\right)\right) \cdot \widehat{T}^{*}$ \\
\hline Miyano $^{75}$ & $\widehat{T}^{*}=T^{*}$ & $\widehat{p}^{*}=Z \cdot \widehat{\rho}^{*} \cdot \widehat{T}^{*}$ \\
\hline [MIYANO] & $\widehat{\rho}^{*}=\rho^{*}$ & $\widehat{u}_{\mathrm{res}}^{*}=u_{\mathrm{r}}^{*} \cdot \widehat{T}^{*}$ \\
\hline Nicolas et al. ${ }^{61}$ & $\widehat{T}^{*}=T^{*}$ & $\widehat{p}^{*}=p^{*}$ \\
\hline \multirow[t]{2}{*}{ [NICOLAS] } & $\widehat{\rho}^{*}=\rho^{*}$ & $\widehat{u}_{\mathrm{res}}^{*}=u_{\mathrm{r}}^{*}$ \\
\hline & & $\widehat{B}=B \cdot \frac{2 \pi}{3}$ \\
\hline Saager and Fischer 69 & $\widehat{T}^{*}=T^{*}$ & $\widehat{p}^{*}=p^{*}$ \\
\hline$[\mathrm{SAAGFI}]$ & $\widehat{\rho}^{*}=\rho^{*}$ & $\widehat{u}_{\mathrm{res}}^{*}=u_{\mathrm{r}}^{*}$ \\
\hline
\end{tabular}

Continued on next page 


\begin{tabular}{|c|c|c|}
\hline $\begin{array}{l}\text { Authors \& } \\
\text { [Acronym] }\end{array}$ & $\begin{array}{l}\text { state point } \\
T, \rho\end{array}$ & $\begin{array}{l}\text { thermophysical } \\
\text { properties }\end{array}$ \\
\hline$K o f k e^{104}$ & $\widehat{T}^{*}=T^{*}$ & $\widehat{p}_{v}^{*}=P$ \\
\hline$[\mathrm{KOFKE}]$ & $\widehat{\rho}^{*}=\rho^{*}$ & $\Delta \widehat{h}_{v}^{*}=\left(u_{1}-\frac{\widehat{p}_{1}^{*}}{\widehat{\rho}_{1}^{*}}+\left(-\widehat{u}_{\mathrm{v}}+\frac{\widehat{p}_{\mathrm{v}}^{*}}{\widehat{\rho}_{\mathrm{v}}^{*}}\right)\right)$ \\
\hline $\operatorname{Adams}^{58}$ & $\widehat{T}^{*}=T^{*}$ & $\widehat{p}^{*}=p^{*}$ \\
\hline \multirow[t]{4}{*}{ [ADAMS3] } & $\widehat{\rho}^{*}=\rho^{*}$ & $\widehat{u}_{\mathrm{res}}^{*}=u_{\mathrm{r}}^{*}$ \\
\hline & & $\widehat{\mu}_{\mathrm{res}}^{*}=\mu_{\mathrm{r}}^{*}$ \\
\hline & & $\Delta \widehat{h}_{v}^{*}=\Delta h_{v}^{*}$ \\
\hline & & $\widehat{p}_{v}^{*}=p_{v}^{*}$ \\
\hline Mecke et al. ${ }^{78}$ & $\widehat{T}^{*}=T^{*}$ & $\widehat{u}_{\mathrm{res}}^{*}=u_{\mathrm{r}}^{*}$ \\
\hline$[\mathrm{MECK}]$ & $\widehat{\rho}^{*}=\rho^{*}$ & $\widehat{p}^{*}=p^{*}$ \\
\hline Potoff and Panagiotopoulos ${ }^{116}$ & $\widehat{T}^{*}=T^{*}$ & $\widehat{\gamma}^{*}=\gamma^{*}$ \\
\hline [POTPAN2] & $\widehat{\rho}^{*}=\rho^{*}$ & \\
\hline Kolafa et al. ${ }^{74}$ & $\widehat{T}^{*}=T^{*}$ & $\widehat{p}^{*}=p^{*}$ \\
\hline \multirow[t]{2}{*}[\text{KOLVOR}]{} & $\widehat{\rho}^{*}=\rho^{*}$ & $\widehat{u}_{\mathrm{res}}^{*}=u_{\mathrm{r}}^{*}$ \\
\hline & & $\widehat{\mu}_{\mathrm{res}}^{*}=\widehat{T}^{*} \cdot \mu_{\mathrm{r}}^{*}$ \\
\hline Emampour et al. ${ }^{157}$ & $\widehat{T}^{*}=T^{*}$ & $\widehat{\Gamma}^{*}=\Gamma^{*}$ \\
\hline$[\mathrm{EMAM}]$ & $\widehat{\rho}^{*}=\rho^{*}$ & \\
\hline Agrawal and Kofke ${ }^{106}$ & $\widehat{T}^{*}=\frac{1}{T^{*}}$ & $\widehat{p}_{v}^{*}=10^{-3} \cdot p^{*}$ \\
\hline \multirow[t]{2}{*}[\mathrm{AGRKOF}]{} & $\widehat{\rho}_{1}^{*}=\rho_{1}^{*}$ & $\Delta \widehat{h}_{v}^{*}=\left(-u_{\mathrm{g}}^{*}+\frac{\widehat{p}^{*}}{\widehat{\rho}_{\mathrm{v}}^{*}}+u_{1}^{*}-\frac{\widehat{p}^{*}}{\widehat{\rho}_{1}^{*}}\right)$ \\
\hline & $\widehat{\rho}_{\mathrm{v}}^{*}=\rho_{\mathrm{g}}^{*} \cdot 10^{-3}$ & \\
\hline Lustig $^{77}$ & $\widehat{T}^{*}=T^{*}$ & $\widehat{p}^{*}=p^{*}$ \\
\hline \multirow[t]{4}{*}{ [LUSTIG] } & $\widehat{\rho}^{*}=\rho^{*}$ & $\widehat{u}_{\mathrm{res}}^{*}=u_{\mathrm{r}}^{*}$ \\
\hline & & $\widehat{\beta}^{*}=\beta^{*}$ \\
\hline & & $\widehat{\mu}_{\mathrm{res}}^{*}=\mu_{\mathrm{r}}^{*}$ \\
\hline & & $\widehat{\gamma}^{*}=\gamma^{*}$ \\
\hline Boda et al. ${ }^{154}$ & $\widehat{T}^{*}=T^{*}$ & $\widehat{c}_{v}^{*}=\frac{3}{2}+c_{v, \mathrm{r}}^{*}$ \\
\hline
\end{tabular}

Continued on next page 


\begin{tabular}{|c|c|c|}
\hline $\begin{array}{l}\text { Authors \& } \\
\text { [Acronym] }\end{array}$ & $\begin{array}{l}\text { state point } \\
T, \rho\end{array}$ & $\begin{array}{l}\text { thermophysical } \\
\text { properties }\end{array}$ \\
\hline$[\mathrm{BODA}]$ & $\widehat{\rho}^{*}=\rho^{*}$ & $\widehat{c}_{p}^{*}=c_{p, \mathrm{r}}^{*}+1.5$ \\
\hline Weeks et al. ${ }^{53}$ & $\widehat{T}^{*}=T^{*}$ & $\widehat{u}_{\mathrm{res}}^{*}=-u_{\mathrm{r}}$ \\
\hline \multirow[t]{2}{*}{ [WEECKCHA2] } & $\hat{\rho}^{*}=\rho^{*}$ & $\widehat{\tilde{a}}_{\mathrm{res}, 00}^{*}=-A_{\mathrm{r}, 00}$ \\
\hline & & $\widehat{p}^{*}=\widehat{\rho}^{*} \cdot \widehat{T}^{*} \cdot \frac{\beta p}{\rho}$ \\
\hline Verlet and Levesque & $\widehat{T}^{*}=T^{*}$ & $\widehat{u}_{\mathrm{res}}^{*}=u_{\mathrm{r}}^{*}$ \\
\hline [VERLEV] & $\widehat{\rho}^{*}=\rho^{*}$ & $\widehat{p}^{*}=\widehat{\rho}^{*} \cdot \widehat{T}^{*} \cdot \frac{\beta p}{\rho}$ \\
\hline Mecke et al. ${ }^{109}$ & $\widehat{T}^{*}=T^{*}$ & $\widehat{\gamma}^{*}=\gamma^{*}$ \\
\hline [MECKWIN] & $\widehat{\rho}^{*}=\rho^{*}$ & \\
\hline Toxvaerd and Praestgaard ${ }^{52}$ & $\widehat{T}^{*}=T^{*}$ & $\widehat{p}^{*}=\widehat{\rho}^{*} \cdot \widehat{T}^{*} \cdot Z$ \\
\hline [TOXPRA] & $\widehat{\rho}^{*}=\rho^{*}$ & \\
\hline Street et al. ${ }^{56}$ & $\widehat{T}^{*}=T^{*}$ & $\widehat{p}^{*}=p^{*}$ \\
\hline [STRRAV] & $\widehat{\rho}^{*}=\rho^{*}$ & $\widehat{u}_{\mathrm{res}}^{*}=u_{\mathrm{r}}^{*}$ \\
\hline Carley $^{59}$ & $\widehat{T}^{*}=T^{*}$ & $\widehat{p}^{*}=\widehat{\rho}^{*} \cdot \widehat{T}^{*} \cdot Z$ \\
\hline [CARL] & $\widehat{\rho}^{*}=\rho^{*}$ & \\
\hline Schofield $^{55}$ & $\widehat{T}^{*}=T^{*}$ & $\widehat{p}^{*}=\rho^{*} \cdot \widehat{T}^{*} \frac{p_{f}}{n \cdot k_{\mathrm{B}} \cdot T^{*}}$ \\
\hline$[\mathrm{SCHOF}]$ & $\widehat{\rho}^{*}=\rho^{*}$ & \\
\hline McDonald and Woodcock 51 & $\widehat{T}^{*}=\frac{T \cdot k_{\mathrm{B}}}{\varepsilon}$ & $\widehat{p}^{*}=\widehat{\rho}^{*} \cdot \widehat{T}^{*} \cdot Z$ \\
\hline \multirow[t]{3}{*}{ [MCDWOO] } & $\widehat{\rho}^{*}=\frac{\rho \cdot N_{\mathrm{AV}} \cdot \sigma^{3} \cdot 10^{-24}}{M}$ & $u_{\mathrm{res}}^{*}=u^{*} \cdot \widehat{T}^{*}$ \\
\hline & & $\frac{\varepsilon}{k_{\mathrm{B}}}=117.2 \mathrm{~K}, \sigma=3.405 \AA$ \\
\hline & & $M=39.948 \frac{\mathrm{g}}{\mathrm{mol}}$ \\
\hline Roccatano et al. ${ }^{79}$ & $\widehat{T}^{*}=T^{*}$ & $\widehat{c}_{v}^{*}=\frac{3}{2}+c_{v, \mathrm{r}}^{*}$ \\
\hline \multirow[t]{2}{*}{ [ROCAMA] } & $\widehat{\rho}^{*}=\rho^{*}$ & $\widehat{u}_{\mathrm{res}}^{*}=u_{\mathrm{r}}^{*}$ \\
\hline & & $\widehat{p}^{*}=p^{*}$ \\
\hline Lustig $^{84}$ & $\widehat{T}^{*}=T^{*}$ & $\widehat{c}_{p}^{*}=c_{p}^{*}$ \\
\hline [LUSTIG2] & $\widehat{\rho}^{*}=\rho^{*}$ & $\widehat{\mu}_{\mathrm{JT}}^{*}=\mu_{\mathrm{JT}}^{*}$ \\
\hline
\end{tabular}

Continued on next page 


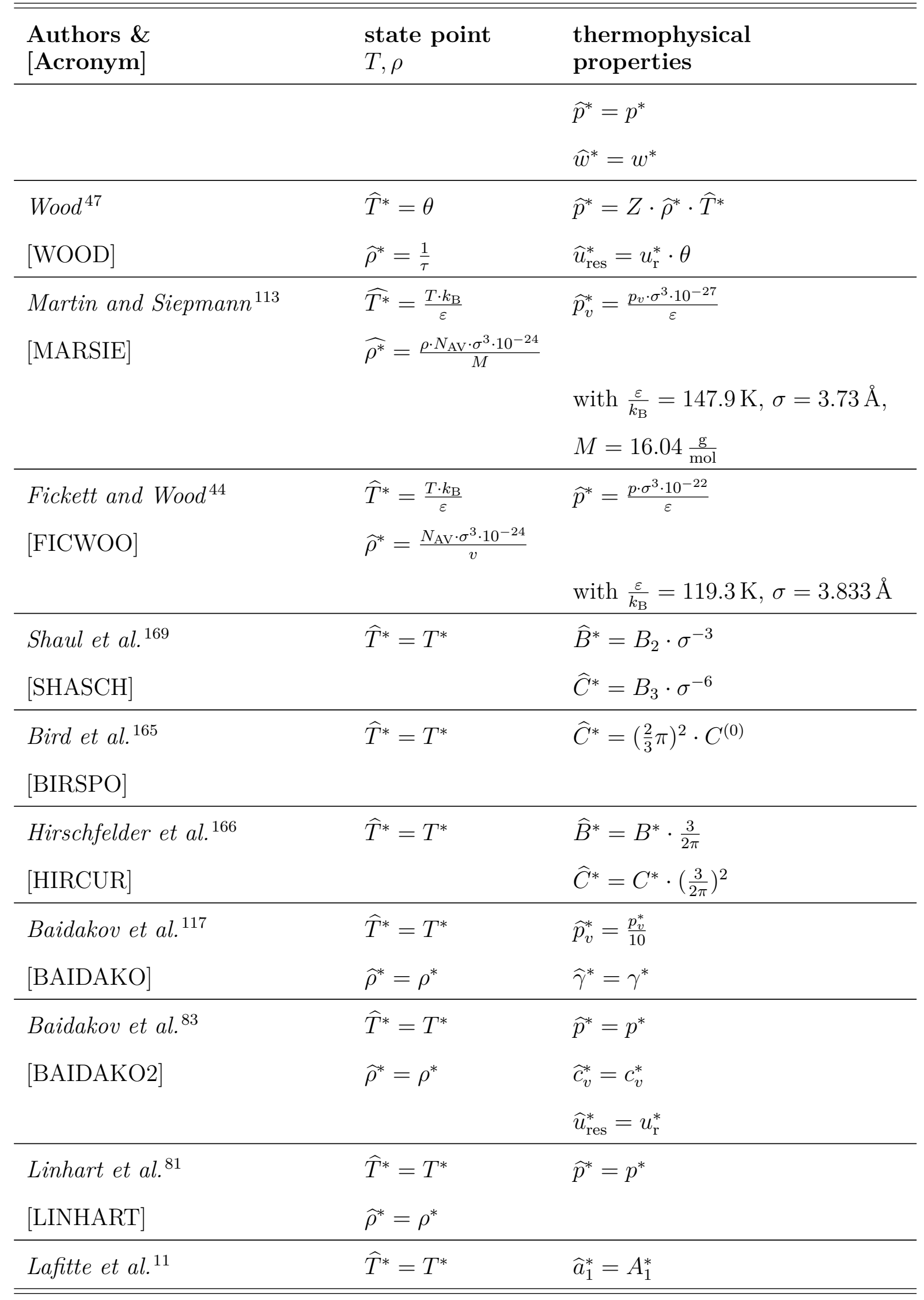

Continued on next page 


\begin{tabular}{lll}
\hline \hline $\begin{array}{l}\text { Authors \& } \\
{[\text { Acronym }]}\end{array}$ & $\begin{array}{l}\text { state point } \\
T, \rho\end{array}$ & $\begin{array}{l}\text { thermophysical } \\
\text { properties }\end{array}$ \\
\hline [LAFITTE] & $\widehat{\rho^{*}}=\rho^{*}$ & $\widehat{a}_{2}^{*}=A_{2}^{*}$ \\
& & $\widehat{a}_{3}^{*}=A_{3}^{*}$
\end{tabular}

Data was digitalized from the corresponding plots from Ref. ${ }^{11}$

\begin{tabular}{|c|c|c|}
\hline Lucas $^{65}$ & $\widehat{T}^{*}=T^{*}$ & $\widehat{p}^{*}=Z \cdot \widehat{\rho}^{*} \cdot \widehat{T}^{*}$ \\
\hline [LUCAS] & $\widehat{\rho^{*}}=\rho^{*}$ & $\widehat{u}_{\mathrm{res}}^{*}=\frac{E}{R \cdot T} \cdot \widehat{T}^{*}$ \\
\hline Betancourt-Cárdenas et al. ${ }^{130}$ & $\widehat{T}^{*}=T^{*}$ & $\widehat{p}^{*}=p^{*}$ \\
\hline [BETANCOU] & $\widehat{\rho}^{*}=\rho^{*}$ & $\begin{array}{l}\widehat{a}_{2}=A_{2}, \quad \widehat{a}_{1}=A_{1} \\
\Delta \widehat{h}_{v}^{*}=u_{\mathrm{v}}^{*}+\frac{\widehat{p}_{\mathrm{v}}^{*}}{\widehat{\rho}_{\mathrm{v}}^{*}}-u_{1}^{*}-\frac{\widehat{p}_{1}^{*}}{\widehat{\rho}_{1}^{*}}\end{array}$ \\
\hline Janeček et al. ${ }^{135}$ & $\widehat{T}^{*}=T^{*}$ & $\widehat{p}_{v}^{*}=p_{v}^{*}$ \\
\hline$[\mathrm{JANECEK}]$ & $\widehat{\rho}^{*}=\rho^{*}$ & $\begin{array}{l}\Delta \widehat{h}_{v}^{*}=\Delta h_{v}^{*} \\
\widehat{\gamma}^{*}=\gamma^{*}\end{array}$ \\
\hline
\end{tabular}

The VLE data from the inhomogeneous simulations were digitalized from the corresponding plots from Ref. ${ }^{135}$ In this work the inhomogeneous simulations-data from Janeček et al. ${ }^{135}$ is referred to as data from Ref. ${ }^{135}$ and [JANECEK]. For the GEMC results from the same publication see [PRIVJANEC].

\begin{tabular}{lll}
\hline Guo et al. ${ }^{111}$ & $\widehat{T}^{*}=T^{*}$ & $\widehat{\gamma}^{*}=\gamma^{*}$ \\
[GUO $]$ & $\widehat{\rho}^{*}=\rho^{*}$ &
\end{tabular}

Surface tension data was digitalized from the corresponding plots from Ref. ${ }^{111}$

\begin{tabular}{lll}
\hline Lee et $a .^{98}$ & $\widehat{T}^{*}=\frac{T \cdot k_{\mathrm{B}}}{\varepsilon}$ & $\widehat{\gamma}^{*}=0.001 \cdot \gamma \sigma^{2} \varepsilon^{-1}$ \\
[LEE] & $\widehat{\rho}^{*}=\rho^{*}$ & \\
& & with $\frac{\varepsilon}{k_{\mathrm{B}}}=119.4 \mathrm{~K}, \sigma=3.4 \AA$ \\
\hline Werth et al. ${ }^{137}$ & $\widehat{T}^{*}=T^{*}$ & $\Delta \widehat{h}_{v}^{*}=\Delta h_{v} *$ \\
{$[$ WERTH] } & $\widehat{\rho}^{*}=\rho^{*}$ & $\widehat{p}_{v}^{*}=p_{v}^{*}$ \\
& & $\widehat{\gamma}^{*}=\gamma^{*}$ \\
\hline Stephan and Hasse ${ }^{138}$ & $\widehat{T}^{*}=T^{*}$ & $\widehat{p}_{v}^{*}=p_{v}^{*}$ \\
\hline \hline
\end{tabular}

Continued on next page 


\begin{tabular}{|c|c|c|}
\hline $\begin{array}{l}\text { Authors \& } \\
\text { [Acronym] }\end{array}$ & $\begin{array}{l}\text { state point } \\
T, \rho\end{array}$ & $\begin{array}{l}\text { thermophysical } \\
\text { properties }\end{array}$ \\
\hline [STEPHAN2] & $\widehat{\rho}^{*}=\rho^{*}$ & \\
\hline Nijmeijer et al. ${ }^{102}$ & $\widehat{T}^{*}=T^{*}$ & $\widehat{\gamma}^{*}=\gamma^{*}$ \\
\hline [NIJMEIJ] & $\widehat{\rho}^{*}=\rho^{*}$ & \\
\hline Errington $^{125}$ & $\widehat{T}^{*}=T^{*}$ & $\widehat{p}_{v}^{*}=p_{v}^{*}$ \\
\hline [ERRING] & $\widehat{\rho}^{*}=\rho^{*}$ & $\widehat{\gamma}^{*}=\gamma^{*}$ \\
\hline Baidakov et al. ${ }^{129}$ & $\widehat{T}^{*}=T^{*}$ & $\widehat{p}_{v}^{*}=p_{v}^{*}$ \\
\hline [BAIDAKOV1] & $\widehat{\rho}^{*}=\rho^{*}$ & $\widehat{\gamma}^{*}=\gamma^{*}$ \\
\hline Janeček $^{131}$ & $\widehat{T}^{*}=T^{*}$ & $\Delta \widehat{h}_{v}^{*}=\Delta h_{v} *$ \\
\hline \multirow[t]{2}{*}[\mathrm{JANECE}]{} & $\widehat{\rho}^{*}=\rho^{*}$ & $\widehat{p}_{v}^{*}=p_{v}^{*}$ \\
\hline & & $\widehat{\gamma}^{*}=\gamma^{*}$ \\
\hline Martinez-Ruiz et al. ${ }^{134}$ & $\widehat{T}^{*}=T^{*}$ & $\widehat{p}_{v}^{*}=p_{v}^{*}$ \\
\hline [MARTINEZ] & $\widehat{\rho}^{*}=\rho^{*}$ & $\widehat{\gamma}^{*}=\gamma^{*}$ \\
\hline Chen et al. ${ }^{24}$ & $\widehat{T}^{*}=T^{*}$ & $\widehat{\gamma}^{*}=\gamma^{*}$ \\
\hline [CHEN] & $\widehat{\rho}^{*}=\rho^{*}$ & \\
\hline Galliero et al. ${ }^{132}$ & $\widehat{T}^{*}=T^{*}$ & $\widehat{\gamma}^{*}=\gamma^{*}$ \\
\hline [GALLIER] & $\widehat{\rho}^{*}=\rho^{*}$ & \\
\hline Trokhymchuk and Alejandre ${ }^{114}$ & $\widehat{T}^{*}=T^{*}$ & $\widehat{p}_{v}^{*}=p_{v}^{*}$ \\
\hline$[\mathrm{TROK}]$ & $\widehat{\rho}^{*}=\rho^{*}$ & $\widehat{\gamma}^{*}=\gamma^{*}$ \\
\hline Anisimov et al. ${ }^{115}$ & $\widehat{T}^{*}=T^{*}$ & $\widehat{p}_{v}^{*}=p_{v}^{*} \cdot 10^{-3}$ \\
\hline [ANISIMOV] & $\widehat{\rho}^{*}=\rho^{*}$ & $\widehat{\gamma}^{*}=\gamma^{*}$ \\
\hline Guo and $L u^{112}$ & $\widehat{T}^{*}=T^{*}$ & $\widehat{\gamma}^{*}=\gamma^{*}$ \\
\hline [GUOLU] & $\widehat{\rho}^{*}=\rho^{*}$ & \\
\hline Mausbach et al. ${ }^{159}$ & $\widehat{T}^{*}=T^{*}$ & $\widehat{\Gamma}^{*}=\Gamma^{*}$ \\
\hline [MAUSVRAB] & $\widehat{\rho}^{*}=\rho^{*}$ & \\
\hline Köster et al. ${ }^{91}$ & $\widehat{T}^{*}=T^{*}$ & $\widehat{\tilde{a}}_{\mathrm{res}, 01}^{*}=A_{\mathrm{r}, 01}^{*}$ \\
\hline
\end{tabular}

Continued on next page 


\begin{tabular}{|c|c|c|}
\hline $\begin{array}{l}\text { Authors \& } \\
\text { [Acronym] }\end{array}$ & $\begin{array}{l}\text { state point } \\
T, \rho\end{array}$ & $\begin{array}{l}\text { thermophysical } \\
\text { properties }\end{array}$ \\
\hline \multirow[t]{4}{*}[\mathrm{KOESTER}]{} & \multirow[t]{4}{*}{$\widehat{\rho}^{*}=\rho^{*}$} & $\widehat{\tilde{a}}_{\mathrm{res}, 11}^{*}=A_{\mathrm{r}, 11}^{*}$ \\
\hline & & $\widehat{\tilde{a}}_{\mathrm{res}, 10}^{*}=A_{\mathrm{r}, 10}^{*}$ \\
\hline & & $\widehat{\tilde{a}}_{\mathrm{res}, 20}^{*}=A_{\mathrm{r}, 20}^{*}$ \\
\hline & & $\widehat{\tilde{a}}_{\mathrm{res}, 02}^{*}=A_{\mathrm{r}, 02}^{*}$ \\
\hline Köster et al. ${ }^{91,92}$ & $\widehat{T}^{*}=T^{*}$ & $\widehat{\tilde{a}}_{\mathrm{res}, 00}^{*}=A_{\mathrm{r}, 00}^{*}$ \\
\hline \multirow[t]{5}{*}{ [KOESTERPRIVCOM] } & \multirow[t]{5}{*}{$\widehat{\rho}^{*}=\rho^{*}$} & $\widehat{\tilde{a}}_{\mathrm{res}, 01}^{*}=A_{\mathrm{r}, 01}^{*}$ \\
\hline & & $\widehat{\tilde{a}}_{\mathrm{res}, 10}^{*}=A_{\mathrm{r}, 10}^{*}$ \\
\hline & & $\widehat{\tilde{a}}_{\mathrm{res}, 11}^{*}=A_{\mathrm{r}, 11}^{*}$ \\
\hline & & $\widehat{\tilde{a}}_{\mathrm{res}, 02}^{*}=A_{\mathrm{r}, 02}^{*}$ \\
\hline & & $\widehat{\tilde{a}}_{\mathrm{res}, 20}^{*}=A_{\mathrm{r}, 20}^{*}$ \\
\hline \multicolumn{3}{|c|}{$\begin{array}{l}\text { Some of the numerical values from Ref. }{ }^{91} \text { were not included in the correspond- } \\
\text { ing supplementary material. This data was made available by the authors and is } \\
\text { included in the database as [KOESTERPRIVCOM] and referred to as data from } \\
\text { Ref. }{ }^{91,92}\end{array}$} \\
\hline Yao et al. ${ }^{63}$ & $\widehat{T}^{*}=T^{*}$ & $\widehat{p}^{*}=p^{*}$ \\
\hline \multirow[t]{2}{*}[\mathrm{YAO}]{} & \multirow[t]{2}{*}{$\widehat{\rho}^{*}=\rho^{*}$} & $\widehat{u}_{\mathrm{res}}^{*}=-U^{*} / N$ \\
\hline & & $\widehat{\mu}_{\mathrm{res}}^{*}=-\mu^{*}-\ln \left(\widehat{\rho}^{*}\right) \cdot T^{*}$ \\
\hline Baidakov et al. ${ }^{123}$ & $\widehat{T}^{*}=T^{*}$ & $\widehat{\rho}^{*}=\rho^{*}$ \\
\hline$[\mathrm{BAIDAKOV} 2]$ & $\widehat{\rho}^{*}=\rho^{*}$ & $\widehat{\gamma}^{*}=\gamma^{*}$ \\
\hline \multicolumn{3}{|c|}{ Data was digitalized from the corresponding plots from Ref. ${ }^{123}$} \\
\hline Hong and Jhon ${ }^{163}$ & $\widehat{T}^{*}=T^{*}$ & $\widehat{\widetilde{a}}_{\mathrm{res}, 00}^{*}=-A_{\mathrm{res}, 00}^{*}$ \\
\hline$[\mathrm{SEUNGMU}]$ & \multicolumn{2}{|l|}{$\widehat{\rho}^{*}=\rho^{*}$} \\
\hline Torrie and Valleau ${ }^{96}$ & $\widehat{T}^{*}=T^{*}$ & $\widehat{u}_{\mathrm{res}}^{*}=u_{\mathrm{r}}^{*}$ \\
\hline$[$ TORVAL $]$ & $\widehat{\rho}^{*}=N / V^{*}$ & $\widehat{\tilde{a}}_{\mathrm{res}, 00}^{*}=\left(\frac{A_{c}}{N \cdot \varepsilon}\right) \cdot \frac{1}{\widehat{T}^{*}}-\ln \left(\widehat{\rho}^{*}\right)+1$ \\
\hline Schultz and Kofke ${ }^{95}$ & $\widehat{T}^{*}=T^{*}$ & $\widehat{p}^{*}=p^{*}$ \\
\hline
\end{tabular}

Continued on next page 


\begin{tabular}{|c|c|c|}
\hline $\begin{array}{l}\text { Authors \& } \\
{[\text { Acronym] }}\end{array}$ & $\begin{array}{l}\text { state point } \\
T, \rho\end{array}$ & $\begin{array}{l}\text { thermophysical } \\
\text { properties }\end{array}$ \\
\hline [SCHULTZKOFKE] & $\widehat{\rho}^{*}=\rho^{*}$ & $\widehat{u}_{\mathrm{res}}^{*}=u_{\mathrm{r}}^{*}$ \\
\hline van Westen and Gross ${ }^{10}$ & $\widehat{T}^{*}=T^{*}$ & $\widehat{a}_{1}^{*}=A_{1}^{*}$ \\
\hline \multirow[t]{2}{*}{ [WESTGROSS] } & $\widehat{\rho}^{*}=\rho^{*}$ & $\widehat{a}_{2}^{*}=A_{2}^{*}$ \\
\hline & & $\widehat{a}_{3}^{*}=A_{3}^{*}$ \\
\hline this work & $\widehat{T}^{*}=T^{*}$ & $\widehat{\tilde{a}}_{\mathrm{res}, 00}^{*}=\tilde{a}_{\mathrm{res}, 00}^{*}$ \\
\hline \multirow[t]{7}{*}{ [STEPHANTHIS] } & $\widehat{\rho}^{*}=\rho^{*}$ & $\widehat{\tilde{a}}_{\mathrm{res}, 01}^{*}=\tilde{a}_{\mathrm{res}, 01}^{*}$ \\
\hline & & $\widehat{\tilde{a}}_{\mathrm{res}, 10}^{*}=\tilde{a}_{\mathrm{res}, 10}^{*}$ \\
\hline & & $\widehat{\tilde{a}}_{\mathrm{res}, 11}^{*}=\tilde{a}_{\mathrm{res}, 11}^{*}$ \\
\hline & & $\widehat{\tilde{a}}_{\mathrm{res}, 02}^{*}=\tilde{a}_{\mathrm{res}, 02}^{*}$ \\
\hline & & $\widehat{\tilde{a}}_{\mathrm{res}, 20}^{*}=\tilde{a}_{\mathrm{res}, 20}^{*}$ \\
\hline & & $p_{v}^{*}=p_{v}^{*}$ \\
\hline & & $\Delta h_{v}^{*}=\Delta h_{v}^{*}$ \\
\hline Errington ${ }^{126,127}$ & $\widehat{T}^{*}=T^{*}$ & $\widehat{p}_{v}^{*}=p_{v}^{*}$ \\
\hline [ERRING2] & $\widehat{\rho}^{*}=\rho^{*}$ & \\
\hline \multicolumn{3}{|c|}{$\begin{array}{l}\text { The data listed as [ERRING2] was forwarded by J. Errington. }{ }^{127} \text { Please note, that } \\
\text { two websites provide numerical values for the LJ VLE. }{ }^{215,216} \text { The website can be } \\
\text { understood in a way that the numeric values might be those of Ref., }{ }^{126} \mathrm{cf} \text {. Ref. }{ }^{10} \\
\text { The numeric values from }{ }^{215,216} \text { are however dissimilar than those from. }{ }^{126,127}\end{array}$} \\
\hline Janeček et al. ${ }^{135,136}$ & $\widehat{T}^{*}=T^{*}$ & $\Delta \widehat{h}_{v}^{*}=\Delta h_{v}^{*}$ \\
\hline [PRIVJANEC] & $\widehat{\rho}^{*}=\rho^{*}$ & $\widehat{p}_{v}^{*}=p_{v}^{*}$ \\
\hline \multicolumn{3}{|c|}{$\begin{array}{l}\text { The numeric values of the VLE data from the GEMC simulations from Ref. }{ }^{135} \text { were } \\
\text { made available by J. Janeček }{ }^{136} \text { and are listed in the database as [PRIVJANEC]. }\end{array}$} \\
\hline Okumura and Yonezawa ${ }^{118,119}$ & $\widehat{T}^{*}=T^{*}$ & $\widehat{p}_{v}^{*}=p_{v}^{*}$ \\
\hline [PRIVOKU] & $\widehat{\rho}^{*}=\rho^{*}$ & \\
\hline Kioupis et al. ${ }^{124}$ & $\widehat{T}^{*}=T^{*}$ & $\widehat{p}_{v}^{*}=p_{\mathrm{sat}}$ \\
\hline [KIOUPIS] & $\widehat{\rho}^{*}=\rho$ & $\Delta \widehat{h}_{v}^{*}=H_{\mathrm{vap}}-H_{\mathrm{liq}}$ \\
\hline
\end{tabular}

Continued on next page 


\begin{tabular}{|c|c|c|}
\hline $\begin{array}{l}\text { Authors \& } \\
\text { [Acronym] }\end{array}$ & $\begin{array}{l}\text { state point } \\
T, \rho\end{array}$ & $\begin{array}{l}\text { thermophysical } \\
\text { properties }\end{array}$ \\
\hline Okumura and Yonezawa ${ }^{121,122}$ & $\widehat{T}^{*}=T^{*}$ & $\widehat{p}_{v}^{*}=p_{v}^{*}$ \\
\hline [PRIVOKU2] & $\widehat{\rho}^{*}=\rho^{*}$ & \\
\hline Mick et al. ${ }^{23}$ & $\widehat{T}^{*}=T^{*}$ & ${\widehat{p^{*}}}_{v}=p_{v}^{*}$ \\
\hline$[\mathrm{MICK}]$ & $\hat{\rho}^{*}=\rho^{*}$ & $\Delta \widehat{h}_{v}^{*}=u_{v}^{*}+\frac{p^{*}}{\hat{\rho}_{v}^{*}}-u_{l}^{*}-\frac{p^{*}}{\hat{\rho}_{l}^{*}}$ \\
\hline Ustinov $^{93,94}$ & $\widehat{T}^{*}=T^{*}$ & $\widehat{p}^{*}=p^{*}$ \\
\hline \multirow[t]{2}{*}{ [PRIVUSTINOV] } & $\widehat{\rho}^{*}=\rho^{*}$ & $\widehat{u}_{\mathrm{res}}^{*}=u_{\mathrm{r}}^{*}$ \\
\hline & & $\widehat{\mu}_{\mathrm{res}}^{*}=\mu^{*}$ \\
\hline Hong and Jang ${ }^{164}$ & $\widehat{T}^{*}=T^{*}$ & $\widehat{\tilde{a}}_{\mathrm{res}, 00}^{*}=-A^{\mathrm{ex}} / N k_{\mathrm{B}} T$ \\
\hline$[\mathrm{HONG}]$ & $\widehat{\rho}^{*}=\rho^{*}$ & \\
\hline Giaquinta et al. ${ }^{72}$ & $\widehat{T}^{*}=T^{*}$ & $\widehat{p}^{*}=\frac{\beta P}{\rho} \cdot \widehat{\rho}^{*} \cdot \widehat{T}^{*}$ \\
\hline [GIAQUI] & $\widehat{\rho}^{*}=\rho^{*}$ & $\widehat{u}_{\mathrm{res}}^{*}=u_{\mathrm{r}}^{*}$ \\
\hline Cuadros et al. ${ }^{162}$ & $\widehat{T}^{*}=T^{*}$ & $\widehat{\widehat{a}}_{\mathrm{res}, 00}^{*}=\frac{A_{\mathrm{r}, 00}}{\widehat{T}^{*}}$ \\
\hline [CUADROS] & $\widehat{\rho}^{*}=\rho^{*}$ & \\
\hline Adachi et al. ${ }^{67}$ & $\widehat{T}^{*}=T^{*}$ & $\widehat{p}^{*}=p^{*}$ \\
\hline$[\mathrm{ADACHI}]$ & $\widehat{\rho}^{*}=\rho^{*}$ & \\
\hline Baranyai et al. ${ }^{68}$ & $\widehat{T}^{*}=T^{*}$ & $\widehat{p}^{*}=p^{*}$ \\
\hline [BARANY] & $\widehat{\rho}^{*}=\rho^{*}$ & $\widehat{u}_{\mathrm{res}}^{*}=u_{\mathrm{r}}^{*}$ \\
\hline & & $\widehat{\tilde{a}}_{\mathrm{res}, 00}^{*}=\frac{A^{\mathrm{ex}} / N}{\widehat{T}^{*}}$ \\
\hline Powles et al. ${ }^{64}$ & $\widehat{T}^{*}=T^{*}$ & $\widehat{p}^{*}=p-1.09 \cdot\left(\widehat{\rho}^{*}\right)^{2}$ \\
\hline [POWLES] & $\widehat{\rho}^{*}=\rho^{*}$ & $\widehat{\mu}_{\mathrm{res}}^{*}=\mu_{1}-\widehat{T}^{*} \cdot \ln \left(\widehat{\rho}^{*}\right)$ \\
\hline & & $+\frac{3}{2} \widehat{T}^{*} \cdot \ln \left(\widehat{T}^{*}\right)-1.86 \widehat{\rho}^{*}$ \\
\hline $\operatorname{Han}^{161}$ & $\widehat{T}^{*}=T^{*}$ & $\widehat{\mu}_{\mathrm{res}}^{*}=\widehat{T}^{*} \cdot \mu_{\mathrm{r}}^{*}$ \\
\hline$[\mathrm{HAN}]$ & $\widehat{\rho}^{*}=\rho^{*}$ & \\
\hline Holcomb et al. ${ }^{105}$ & $\widehat{T}^{*}=T^{*}$ & $\widehat{\gamma}^{*}=\gamma^{*}$ \\
\hline$[\mathrm{HOLCOMB}]$ & $\widehat{\rho}^{*}=\widehat{\rho}^{*}$ & \\
\hline
\end{tabular}

Continued on next page 


\begin{tabular}{|c|c|c|}
\hline $\begin{array}{l}\text { Authors \& } \\
\text { [Acronym] }\end{array}$ & $\begin{array}{l}\text { state point } \\
T, \rho\end{array}$ & $\begin{array}{l}\text { thermophysical } \\
\text { properties }\end{array}$ \\
\hline Stoll et al. ${ }^{128}$ & $\widehat{T}^{*}=\frac{T^{*}}{4}$ & $\widehat{p}_{v}^{*}=\frac{1}{4} p_{v}^{*}$ \\
\hline [STOLL] & $\widehat{\rho}^{*}=\widehat{\rho}^{*}$ & $\Delta \widehat{h}_{v}^{*}=\frac{h_{v, v a p}-h_{v, l i q}}{4}$ \\
\hline Deiters and Neumaier ${ }^{43,97}$ & $\widehat{T}^{*}=\frac{T \cdot k_{\mathrm{B}}}{\varepsilon}$ & $\widehat{p}^{*}=\frac{p^{*} \cdot 10^{-24} \cdot \sigma^{3}}{\left(\varepsilon / k_{\mathrm{B}}\right) \cdot k_{\mathrm{B}}}$ \\
\hline$[\mathrm{DEINEU}]$ & $\widehat{\rho}^{*}=\frac{10^{-24} \cdot N_{\mathrm{AV}} \cdot \sigma^{3}}{V_{M}}$ & $\begin{array}{l}\widehat{u}_{\mathrm{res}}^{*}=\frac{u_{\mathrm{m}}}{\left(\varepsilon / k_{\mathrm{B}}\right) \cdot k_{\mathrm{B}} \cdot N_{\mathrm{AV}}} \\
\text { with } \frac{\varepsilon}{k_{\mathrm{B}}}=119.8 \mathrm{~K}, \sigma=3.405 \AA\end{array}$ \\
\hline Sun and Teja ${ }^{168}$ & $\widehat{T}^{*}=T^{*}$ & $\widehat{B}^{*}=\frac{2}{3} \pi \cdot B_{2}^{*}$ \\
\hline [SUNTEJA] & & $\widehat{C}^{*}=\left(\frac{2}{3} \pi\right)^{2} \cdot B_{3}^{*}$ \\
\hline Sadus and Prausnitz ${ }^{108}$ & $\widehat{T}^{*}=T^{*}$ & $\widehat{p}_{v}^{*}=P^{*}$ \\
\hline [SADUS] & $\widehat{\rho}^{*}=\rho^{*}$ & $\Delta \widehat{h}_{v}^{*}=\left(E_{\mathrm{v}}^{*}+\frac{\widehat{p}_{\mathrm{v}}^{*}}{\widehat{\rho}_{\mathrm{v}}^{*}}-\left(E_{1}^{*}+\frac{\widehat{p}_{1}^{*}}{\widehat{\rho}_{1}^{*}}\right)\right)$ \\
\hline Yigzawe and Sadus ${ }^{87}$ & $\widehat{T}^{*}=\varphi \cdot 1.312$ & $\widehat{p}^{*}=p^{*}$ \\
\hline [YIGSAD] & $\widehat{\rho}^{*}=\rho^{*}$ & \\
\hline Yigzawe 86 & $\widehat{T}^{*}=T^{*}$ & $\widehat{p}_{v}^{*}=p^{*}$ \\
\hline \multirow[t]{8}{*}{ [YIGZAWE] } & $\widehat{\rho}^{*}=\rho^{*}$ & $\widehat{u}^{*}=u^{*}-\frac{3}{2} \widehat{T}^{*}$ \\
\hline & & $\widehat{c}_{p}^{*}=C_{p}^{*}$ \\
\hline & & $\widehat{c}_{v}^{*}=C_{v}^{*}$ \\
\hline & & $\widehat{\alpha}^{*}=\alpha_{\mathrm{P}}^{*}$ \\
\hline & & $\widehat{\beta}^{*}=\beta_{\mathrm{T}}^{*}$ \\
\hline & & $\widehat{\gamma}^{*}=\gamma_{\mathrm{V}}^{*}$ \\
\hline & & $\widehat{\mu}_{\mathrm{JT}}^{*}=\mu_{\mathrm{JT}}^{*}$ \\
\hline & & $\widehat{w}^{*}=w_{0}^{*}$ \\
\hline Mairhofer and Sadus ${ }^{88,89}$ & $\widehat{T}^{*}=T^{*}$ & $\widehat{p}_{v}^{*}=p^{*}$ \\
\hline \multirow[t]{4}{*}[\mathrm{PRIVMAI}]{} & $\widehat{\rho}^{*}=\rho^{*}$ & $\widehat{u}^{*}=u^{*}-\frac{3}{2} \widehat{T}^{*}$ \\
\hline & & $\widehat{c}_{p}^{*}=C_{p}^{*}$ \\
\hline & & $\widehat{c}_{v}^{*}=C_{v}^{*}$ \\
\hline & & $\widehat{\beta}^{*}=\beta_{\mathrm{T}}^{*}$ \\
\hline
\end{tabular}

Continued on next page 


\begin{tabular}{|c|c|c|}
\hline $\begin{array}{l}\text { Authors \& } \\
{[\text { Acronym }]}\end{array}$ & $\begin{array}{l}\text { state point } \\
T, \rho\end{array}$ & $\begin{array}{l}\text { thermophysical } \\
\text { properties }\end{array}$ \\
\hline & & $\begin{array}{l}\widehat{\gamma}^{*}=\gamma^{*} \\
\widehat{\mu}_{\mathrm{JT}}^{*}=\mu_{\mathrm{JT}}^{*} \\
\widehat{w}^{*}=w_{0}^{*}\end{array}$ \\
\hline Plačkov and Sadus ${ }^{110}$ & & $\widehat{p}_{v}^{*}=P^{*}$ \\
\hline$[\mathrm{PLACKOV}]$ & $\widehat{\rho}^{*}=\rho^{*}$ & $\Delta \widehat{h}_{v}^{*}=\left(E_{\mathrm{v}}^{*}+\frac{\widehat{p}_{\mathrm{v}}^{*}}{\widehat{\rho}_{\mathrm{v}}^{*}}-\left(E_{1}^{*}+\frac{\widehat{p}_{1}^{*}}{\widehat{\rho}_{1}^{*}}\right)\right)$ \\
\hline $\begin{array}{l}\text { Sadus }^{133} \\
{[\text { SADUS2] }}\end{array}$ & $\widehat{T}^{*}=T^{*}$ & $\widehat{p}_{v}^{*}=p^{*}$ \\
\hline $\begin{array}{l}\text { Chapela et al. }{ }^{99} \\
{[\text { CHAPELA }]}\end{array}$ & $\widehat{T}^{*}=T^{*}$ & $\widehat{\gamma}^{*}=\gamma^{*}$ \\
\hline $\begin{array}{l}\text { Shen et al. }{ }^{141} \\
{[\mathrm{SHEN}]}\end{array}$ & $\widehat{T}^{*}=T^{*}$ & $\widehat{\gamma}^{*}=\gamma^{*}$ \\
\hline $\begin{array}{l}\text { Werth et al. }{ }^{142} \\
\text { [WERHOR] }\end{array}$ & $\widehat{T}^{*}=T^{*}$ & $\widehat{\gamma}^{*}=\gamma^{*}$ \\
\hline $\begin{array}{l}\text { Miyazaki et al. }{ }^{139} \\
\text { [MIYAZ] }\end{array}$ & $\widehat{T}^{*}=T^{*}$ & $\widehat{\gamma}^{*}=0.001 \cdot \gamma \sigma^{2} \varepsilon^{-1}$ \\
\hline & & with $\frac{\varepsilon}{k_{\mathrm{B}}}=119.8 \mathrm{~K}, \sigma=3.405 \AA$ \\
\hline
\end{tabular}

\section{Details on the assessment of homogeneous states data}

Fig. S1 shows the results of the EOS test on the homogeneous state points as the percentage of the overall confirmed data as a function of the parameter $P_{\max }$. The chosen value of $P_{\max }=4$ is indicated, which entails approximately $90 \%$ confirmed data. For smaller $P_{\max }$ values, the EOS test becomes more stringent as more data points are characterized as outliers. For larger $P_{\max }$ values, the EOS test becomes more lenient (i.e. more conservative) and less data points are identified as outliers. Evidently, the EOS test converges for large $P_{\max }$ values to approximately $N_{\text {conf }}=98 \%$, 
which means that these $2 \%$ of all homogeneous data are particularly gross outliers.

\section{Details on the calculation of thermophysical properties by the Lustig-formalism}

The Lustig formalism in combination with Widom's test particle insertion method, ${ }^{176}$ as implemented in the simulation package $m s 2,{ }^{177}$ provides the Helmholtz energy and its derivatives with respect to the density and the inverse temperature simultaneously from a single simulation run. The Helmholtz energy per particle $a=A / N$ is formally split into an ideal gas contribution (superscript o) and a residual contribution (superscript res), i.e. $a=a^{\mathrm{o}}+a^{\text {res }}$. The following notation is used for the derivatives of the Helmholtz energy $\tilde{a}=a / T$ with respect to inverse temperature and density

$$
\tilde{a}_{n m}=\tilde{a}_{\mathrm{nm}}^{\mathrm{o}}+\tilde{a}_{\mathrm{nm}}^{\mathrm{res}}=(1 / T)^{n} \rho^{m} \frac{\partial^{n+m}\left(\tilde{a}^{\mathrm{o}}+\tilde{a}^{\mathrm{res}}\right)}{\partial(1 / T)^{n} \partial \rho},
$$

where only $n, m=0,1,2$ and were considered here. The numerical values of the $\tilde{a}_{n m}$ obtained in the studies of Refs. ${ }^{90-92}$ and from this work are included in the database, as such are the primary simulation data. The relations used for the computation of thermodynamic properties from the sampled $\tilde{a}_{n m}$ values are as follows:

entropic properties

Gibbs energy $g=h-T s=T\left(1+\tilde{a}_{00}^{\mathrm{o}}+\tilde{a}_{00}^{\mathrm{res}}+\tilde{a}_{01}^{\mathrm{res}}\right)$

thermal properties

pressure

$$
p=-\left(\frac{\partial a}{\partial v}\right)_{T}=\rho T\left(1+\tilde{a}_{01}^{\mathrm{res}}\right)
$$


thermal pressure coefficient $\quad \gamma=\left(\frac{\partial p}{\partial T}\right)_{\rho}=\rho\left(1+\tilde{a}_{01}^{\text {res }}-\tilde{a}_{11}^{\text {res }}\right)$

isothermal compressibility $\quad \beta=\left(\rho \frac{\partial p}{\partial \rho}\right)_{T}^{-1}=\frac{1}{\rho T\left(1+2 \tilde{a}_{01}^{\text {res }}+\tilde{a}_{02}^{\text {res }}\right)}$

$\begin{aligned} & \text { thermal expansion coeffi- } \\ & \text { cient }\end{aligned} \quad \alpha=\beta \gamma=\frac{\left(\frac{\partial p}{\partial T}\right)_{\rho}}{\rho\left(\frac{\partial p}{\partial \rho}\right)_{T}}=\frac{1+\tilde{a}_{01}^{\text {res }}-\tilde{a}_{11}^{\text {res }}}{T\left(1+2 \tilde{a}_{01}^{\text {res }}+\tilde{a}_{02}^{\text {res }}\right)}$

caloric properties

internal energy

$$
\begin{aligned}
& u=a+T s=T\left(\tilde{a}_{10}^{0}+\tilde{a}_{10}^{\mathrm{res}}\right) \\
& u_{\mathrm{res}}=a_{\mathrm{res}}+T s_{\mathrm{res}}=T \tilde{a}_{10}^{\mathrm{res}}
\end{aligned}
$$

enthalpy

$$
h=u+p v=T\left(1+\tilde{a}_{10}^{\mathrm{o}}+\tilde{a}_{10}^{\text {res }}+\tilde{a}_{01}^{\text {res }}\right)
$$

$$
\text { isobaric heat capacity } \quad c_{p}=\left(\frac{\partial h}{\partial T}\right)_{p}=-\left(\tilde{a}_{20}^{\mathrm{o}}+\tilde{a}_{20}^{\mathrm{res}}\right)+\frac{\left(1+\tilde{a}_{01}^{\mathrm{res}}-\tilde{a}_{11}^{\mathrm{res}}\right)^{2}}{1+2 \tilde{a}_{01}^{\mathrm{res}}+\tilde{a}_{02}^{\mathrm{res}}}
$$


isochoric heat capacity $\quad c_{v}=\left(\frac{\partial u}{\partial T}\right)_{v}=-\left(\tilde{a}_{20}^{\mathrm{o}}+\tilde{a}_{20}^{\mathrm{res}}\right)$
$w=\sqrt{\left(\frac{\partial p}{\partial \rho}\right)_{s}}=\left(T\left(1+2 \tilde{a}_{01}^{\mathrm{res}}+\tilde{a}_{02}^{\mathrm{res}}\right)-\frac{\left(1+\tilde{a}_{01}^{\mathrm{res}}-\tilde{a}_{11}^{\mathrm{res}}\right)^{2}}{\tilde{a}_{20}^{\mathrm{o}}+\tilde{a}_{20}^{\mathrm{res}}}\right)^{0.5}$
speed of sound

Joule-Thomson coefficient $\quad \mu_{\mathrm{JT}}=\left(\frac{\partial T}{\partial p}\right)_{h}$

$=\rho^{-1} \frac{-\left(\tilde{a}_{01}^{\text {res }}+\tilde{a}_{02}^{\text {res }}+\tilde{a}_{11}^{\text {res }}\right)}{\left(1+\tilde{a}_{01}^{\text {res }}-\tilde{a}_{11}^{\text {res }}\right)^{2}-\left(\tilde{a}_{20}^{\mathrm{o}}+\tilde{a}_{20}^{\text {res }}\right)\left(1+2 \tilde{a}_{01}^{\text {res }}+\tilde{a}_{02}^{\text {res }}\right)}$

Grüneisen coefficient

$$
\Gamma=\frac{\left(\frac{\partial p}{\partial T}\right)_{\rho}}{\rho c_{v}}=\frac{1+\tilde{a}_{01}^{\mathrm{res}}-\tilde{a}_{11}^{\mathrm{res}}}{-\tilde{a}_{20}^{\mathrm{o}}-\tilde{a}_{20}^{\mathrm{res}}}
$$

\section{Details on the assessment of VLE bulk data}

The results of the three VLE tests for all data sets (both confirmed and discarded) are shown in Figs. S2, S3, and S4 for the sake of completeness. Fig. S2 of the supplementary material shows the results of the compressibility factor test for all VLE data, Fig. S3 shows the results of the Clausius-Clapeyron test for all VLE data, and Fig. S4 shows the deviations from all VLE data and the base correlations (6) - (9).

Data sets that were discarded according the criteria for the compressibility factor test (cf. Fig. S2) outlined in the main part of this work are those from Adams, ${ }^{58}$ Adams,${ }^{60}$ Anisimov 
et al., ${ }^{115}$ Baidakov et al., ${ }^{117}$ Baidakov et al. ${ }^{129}$ Betancourt-Cárdenas et al. ${ }^{130}$ Kioupis et al. ${ }^{124}$ Martin and Siepmann, ${ }^{113}$ Panagiotopoulos, ${ }^{100}$ Panagiotopoulos et al. ${ }^{101}$ Smit and Frenkel, ${ }^{103}$ and Trokhymchuk and Alejandre. ${ }^{114}$

For the data sets from the literature that report all VLE properties required for the ClausiusClapeyron test, the RHS of Eq. (10) was computed and shown in Fig. S3 for comparison. Large deviations from the base correlation and the most precise data sets (Refs. ${ }^{23,71,135,136}$ and this work) were found for the data of Adams, ${ }^{58}$ Adams, ${ }^{60}$ Kioupis et al., ${ }^{124}$ Betancourt-Cárdenas et al. ${ }^{130}$ Panagiotopoulos, ${ }^{100}$ Panagiotopoulos et al., ${ }^{101}$ and Smit and Frenkel. ${ }^{103}$ According the criteria outlined in the main part of this work, these data sets were discarded.

Fig. S4 shows the deviation plots for each VLE property $\left(p^{s}, \rho^{\prime}, \rho^{\prime \prime}\right.$, and $\left.\Delta h_{\mathrm{v}}\right)$ for all VLE data sets considered in this work. The following data sets contain two or more data points with deviations larger than 5\%, i.e. are out of the range of Fig. S4: Adams, ${ }^{58}$ Adams, ${ }^{60}$ Anisimov et al., ${ }^{115}$ Baidakov et al., ${ }^{117}$ Baidakov et al. ${ }^{129}$ Baidakov et al., ${ }^{123}$ Betancourt-Cárdenas et al. ${ }^{130}$ Guo et al., ${ }^{111}$ Guo and Lu, ${ }^{112}$ Galliero et al., ${ }^{132}$ Hunter and Reinhardt, ${ }^{107}$ Holcomb et al. ${ }^{105}$ Janeček, ${ }^{131}$ Kioupis et al., ${ }^{124}$ Kofke,${ }^{104}$ Lee et al., ${ }^{98}$ Martin and Siepmann, ${ }^{113}$ Mecke et al.., ${ }^{109}$ Okumura and Yonezawa, ${ }^{118,119}$ Panagiotopoulos, ${ }^{100}$ Panagiotopoulos et al., ${ }^{101}$ Potoff and Panagiotopoulos, ${ }^{197}$ Smit and Frenkel, ${ }^{103}$ and Trokhymchuk and Alejandre. ${ }^{114}$ These pronounced deviations were found in most cases for the vapor pressure and the saturated vapor density. Deviations of data points that exceed $5 \%$ in the saturated liquid density or the enthalpy of vaporization were only found for the data from Panagiotopoulos, ${ }^{100}$ Smit and Frenkel, ${ }^{103}$ Kofke,${ }^{104}$ Kioupis et al.,${ }^{124}$ Hunter and Reinhardt, ${ }^{107}$ Mecke et al., ${ }^{109}$ and Lee et al. ${ }^{98}$ Data sets exhibiting particularly large deviations from Eqs. (6) - (9) at multiple state points are those from Anisimov et al., ${ }^{115}$ Baidakov et al., ${ }^{117}$ Baidakov et al. ${ }^{129}$ Galliero et al. ${ }^{132}$ Hunter and Reinhardt, ${ }^{107}$ Kioupis et al., ${ }^{124}$ Lee et al., ${ }^{98}$ Panagiotopoulos, ${ }^{100}$ and Trokhymchuk and Alejandre. ${ }^{114}$ To avoid visual clutter, these outof-range data points are not shown in Fig. S4. This confirms the findings from the compressibility factor and the Clausius-Clapeyron tests where these data sets could be applied.

The data sets of Hunter and Reinhardt, ${ }^{107}$ Potoff and Panagiotopoulos, ${ }^{197}$ Shi and Johnson ${ }^{120}$ (only saturated densities reported) show small, but distinct systematic deviations to the base 
correlation and the seven most precise data sets.

\section{Details on the assessment of VLE interfacial data}

Fig. S5 shows the surface tension of all considered data in this work. The surface tension results reported by Trokhymchuk and Alejandre ${ }^{114}$ and the MC results of Galliero et al. ${ }^{132}$ are slightly but noticeably below the mutually best agreeing data sets. The data of Anisimov et al. ${ }^{115}$ show large deviations, which is likely a result of the employed LJ potential version.

The surface tension data of Potoff and Panagiotopoulos ${ }^{116}$ shows distinct deviations from the above mentioned data of best mutual agreement - especially close to the critical point. The same was found for the saturated densities data of Potoff and Panagiotopoulos ${ }^{116}$. This is in line with the relatively low critical temperature reported by Ref.. ${ }^{116}$ 
Table S4 summarizes the identified outliers in the data sets that were identified to be the best VLE data sets: Errington, ${ }^{126,127}$ Janeček et al. ${ }^{135,136}$ Lotfi et al., ${ }^{71}$ Mick et al. ${ }^{23}$ Okumura and Yonezawa ${ }^{121,122}$ and this work. 
Table S3 summarizes the results for the vapor-liquid equilibrium data obtained in this work using the Grand Equilibrium method ${ }^{179}$ as implemented in $m s 2 .{ }^{177}$

Table S3: Simulation results for the VLE data set obtained in this work. The columns are from left to right: vapor pressure, saturated liquid density, saturated vapor density, and the enthalpy of vaporization. The numbers in parentheses indicate the uncertainties of the last decimal digits.

\begin{tabular}{|c|c|c|c|c|}
\hline$T$ & $p^{s}$ & $\rho^{\prime}$ & $\rho^{\prime \prime}$ & $\Delta h_{v}$ \\
\hline 0.69 & $0.001172(24)$ & $0.847111(1)$ & $0.001729(35)$ & $6.766422(34)$ \\
\hline 0.7 & $0.001343(25)$ & $0.8427(1)$ & $0.001956(36)$ & $6.762403(39)$ \\
\hline 0.72 & $0.001784(28)$ & $0.834385(1)$ & $0.002538(39)$ & $6.652809(43)$ \\
\hline 0.74 & $0.002319(29)$ & $0.825751(1)$ & $0.003227(40)$ & $6.641588(48)$ \\
\hline 0.76 & $0.002932(32)$ & $0.817195(2)$ & $0.003994(44)$ & $6.528674(58)$ \\
\hline 0.78 & $0.003689(40)$ & $0.8083(1)$ & $0.004929(54)$ & $6.513863(71)$ \\
\hline 0.8 & $0.004649(44)$ & $0.799470(2)$ & $0.006103(57)$ & $6.395171(72)$ \\
\hline 0.82 & $0.005608(44)$ & $0.7903(1)$ & $0.007233(57)$ & $6.278050(94)$ \\
\hline 0.84 & $0.006872(44)$ & $0.7812(1)$ & $0.008730(55)$ & $6.25512(12)$ \\
\hline 0.86 & $0.008299(53)$ & $0.7718(1)$ & $0.010397(66)$ & $6.13070(14)$ \\
\hline 0.88 & $0.009902(63)$ & $0.7623(1)$ & $0.012248(78)$ & $6.10391(17)$ \\
\hline 0.9 & $0.011795(39)$ & $0.7527(1)$ & $0.014435(48)$ & $5.97235(16)$ \\
\hline 0.92 & $0.013783(67)$ & $0.7427(1)$ & $0.016686(81)$ & $5.84091(21)$ \\
\hline 0.94 & $0.016237(79)$ & $0.7328(1)$ & $0.01952(10)$ & $5.80088(26)$ \\
\hline 0.96 & $0.018686(63)$ & $0.7224(1)$ & $0.02228(07)$ & $5.66382(30)$ \\
\hline 0.98 & $0.021625(75)$ & $0.7120(1)$ & $0.02567(09)$ & $5.51804(44)$ \\
\hline 1 & $0.024843(87)$ & $0.7009(1)$ & $0.02938(10)$ & $5.46904(50)$ \\
\hline 1.02 & $0.02823(10)$ & $0.6899(1)$ & $0.03329(12)$ & $5.31864(67)$ \\
\hline 1.04 & $0.03216(11)$ & $0.6785(1)$ & $0.03795(13)$ & $5.15826(69)$ \\
\hline 1.06 & $0.03623(09)$ & $0.6664(1)$ & $0.04277(11)$ & 4.9978(11) \\
\hline 1.08 & $0.04058(10)$ & $0.6537(2)$ & $0.04799(11)$ & $4.8332(12)$ \\
\hline
\end{tabular}

Continued on next page 


\begin{tabular}{lllll}
\hline \hline$T$ & $p^{s}$ & $\rho^{\prime}$ & $\rho^{\prime \prime}$ & $\Delta h_{v}$ \\
\hline 1.1 & $0.04568(12)$ & $0.6409(2)$ & $0.05443(14)$ & $4.7536(16)$ \\
1.12 & $0.05112(13)$ & $0.6275(2)$ & $0.06145(15)$ & $4.5688(18)$ \\
1.14 & $0.05674(16)$ & $0.6133(2)$ & $0.06885(19)$ & $4.3817(24)$ \\
1.16 & $0.06323(20)$ & $0.5985(2)$ & $0.07810(24)$ & $4.1717(33)$ \\
1.18 & $0.06957(21)$ & $0.5820(2)$ & $0.08715(26)$ & $3.9681(39)$ \\
1.2 & $0.07684(25)$ & $0.5639(3)$ & $0.09862(32)$ & $3.7339(59)$ \\
1.22 & $0.08429(33)$ & $0.5446(4)$ & $0.11101(44)$ & $3.3941(87)$ \\
1.24 & $0.09260(36)$ & $0.5235(5)$ & $0.12657(50)$ & $3.122(11)$ \\
1.26 & $0.10129(44)$ & $0.5007(5)$ & $0.14451(62)$ & $2.823(16)$ \\
1.28 & $0.11082(39)$ & $0.4747(8)$ & $0.17041(60)$ & $2.350(21)$ \\
\hline \hline
\end{tabular}


Table S4: Identified outliers in the best VLE data sets: Errington, ${ }^{126,127}$ Janeček et al., ${ }^{135,136}$ Lotfi et al.,${ }^{71}$ Mick et al. ${ }^{23}$ Okumura and Yonezawa, ${ }^{121,122}$ and this work.

\begin{tabular}{rrllll}
\hline \hline Reference & $T$ & $p^{s}$ & $\rho^{\prime}$ & $\rho^{\prime \prime}$ & $\Delta h_{v}$ \\
\hline Mick et al. $^{23}$ & 0.75 & $0.0022(1)$ & $0.8208(2)$ & $0.0030(1)$ & $6.595(3)$ \\
& 1.25 & $0.0967(4)$ & $0.514(2)$ & $0.135(2)$ & $3.30(3)$ \\
this work $^{(\mathrm{a})}$ & 1.08 & $0.040583(62)$ & $0.65371(20)$ & $0.047992(74)$ & $4.8332(12)$ \\
Lotfi et al. $^{71}$ & 0.7 & $0.00131(6)$ & $0.84266(18)$ & $0.00193(10)$ & $6.758(4)$ \\
Errington $^{125}$ & 1.3 & $0.1212(10)$ & $0.4271(13)$ & $0.2096(13)$ & - \\
Errington $^{126,127}$ & 1.3 & $0.1215841(996)$ & $0.4442(51)$ & $0.193072(5552)$ & - \\
& 1.25 & $0.0975052(672)$ & $0.5049(12)$ & $0.143990(335)$ & - \\
\hline \hline
\end{tabular}

(a) data point is slightly off the statistical uncertainties regarding the self-consistency Clausius-Clapeyron test (see Fig. S3). 


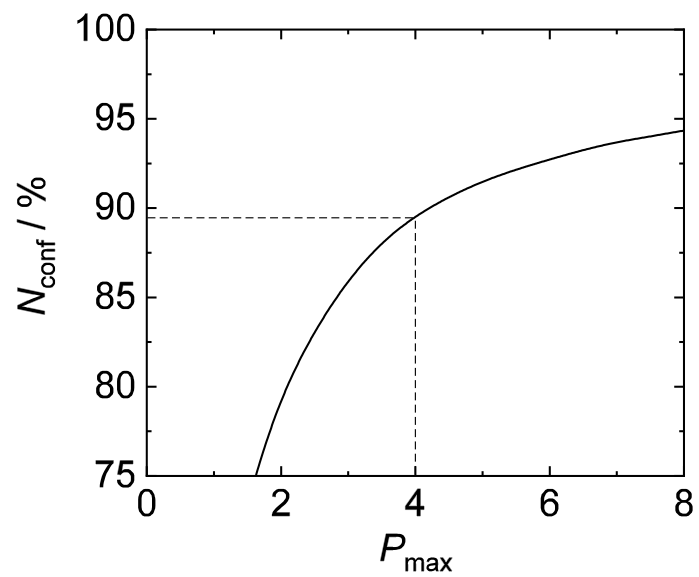

Figure S1: Percentage of overall confirmed data as a function of the parameter $P_{\max }$ for the homogeneous data EOS test. $N_{\text {conf }}$ is the confirmation rate of all homogeneous data. The dashed line indicates the value $P_{\max }=4$ chosen for the identification of outliers as they are specified in the database. 


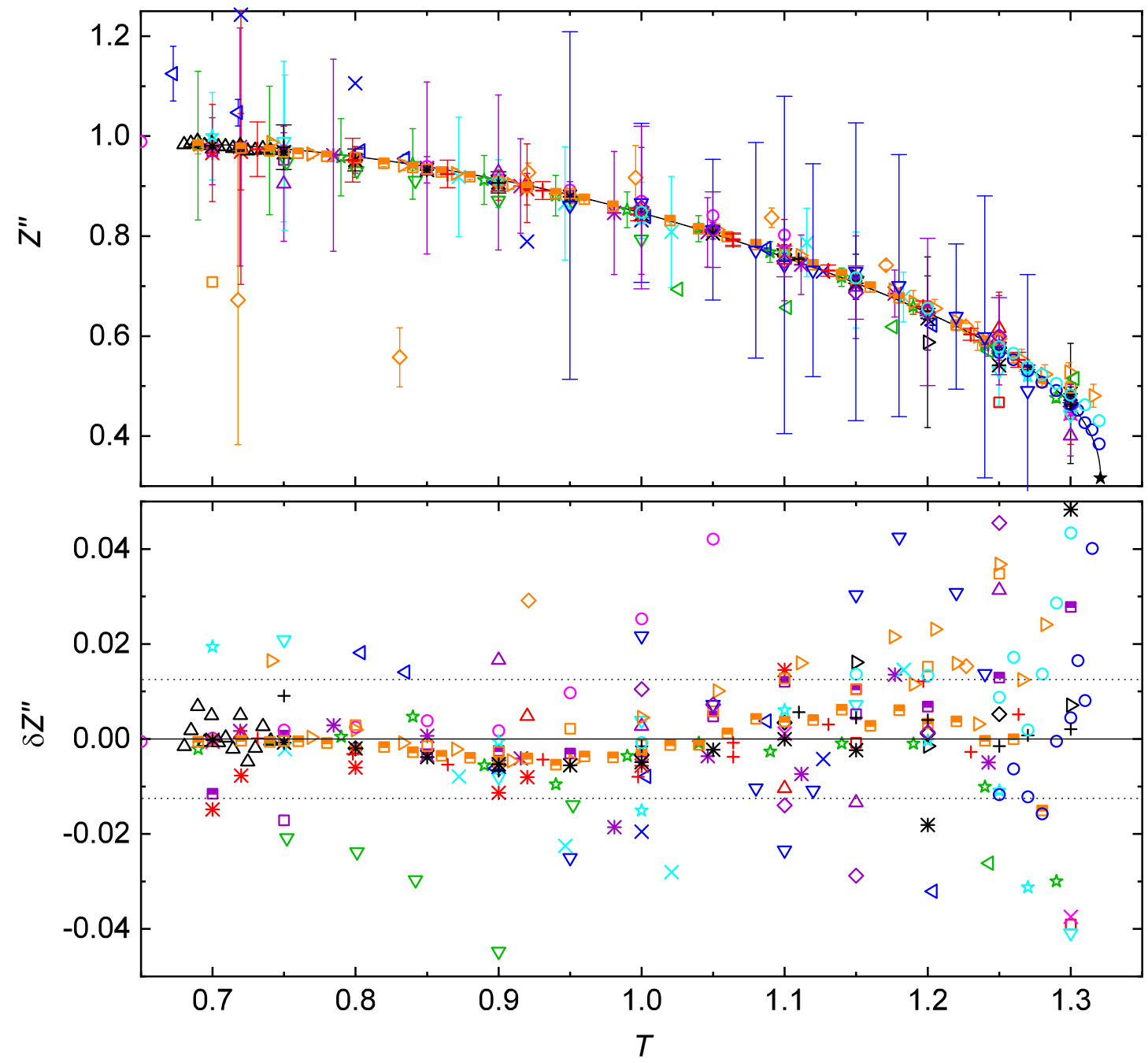

\begin{tabular}{|c|c|c|c|c|c|}
\hline & correlation Eq. (6) \& (8) & ○ & Hunter \& Reinhardt ${ }^{107}$ & $\Delta$ & Panagiotopoulos ${ }^{100}$ \\
\hline$\square$ & Adams $^{60}$ & $\Delta$ & Janecek $^{131}$ & $\nabla$ & Panagiotopoulos et al. ${ }^{101}$ \\
\hline 0 & Adams $^{58}$ & $\diamond$ & Janecek et al. $^{135,136}$ & $\nabla$ & Plackov \& Sadus ${ }^{110}$ \\
\hline$\Delta$ & Agrawal \& Kofke ${ }^{106}$ & $\triangleleft$ & Kioupis et al. ${ }^{124}$ & $\diamond$ & Sadus \& Prausnitz ${ }^{108}$ \\
\hline$\nabla$ & Anisimov et al. ${ }^{115}$ & $\triangleright$ & Kofke ${ }^{104}$ & $\triangleright$ & Smit \& Frenkel ${ }^{103}$ \\
\hline$\diamond$ & Baidakov et al. ${ }^{117}$ & - & Lotfi et al. ${ }^{71}$ & « & Stephan \& Hasse ${ }^{138}$ \\
\hline$\triangleleft$ & Baidakov et al. ${ }^{129}$ & $x$ & Martin \& Siepmann ${ }^{113}$ & + & Stoll et al. ${ }^{128}$ \\
\hline मे & Betancourt-Cardenas et al. ${ }^{130}$ & * & Martinez-Ruiz et al. ${ }^{134}$ & a & this work \\
\hline$\times$ & Errington ${ }^{125}$ & + & Mick et al. ${ }^{23}$ & $x$ & Trokhymchuk \& Alejandre ${ }^{114}$ \\
\hline * & Errington ${ }^{126,127}$ & 口 & Okumura \& Yonezawa ${ }^{118,119}$ & * & Werth et al. ${ }^{137}$ \\
\hline व & Hansen \& Verlet ${ }^{20}$ & 0 & Okumura \& Yonezawa ${ }^{121,122}$ & & \\
\hline
\end{tabular}

Figure S2: Compressibility factor test for the vapor-liquid equilibrium data of the Lennard-Jones fluid according to Nezbeda: ${ }^{213,214}$ saturated vapor phase compressibility factor $Z^{\prime \prime}$ as function of the temperature $T$ (top) and the relative deviation of $Z^{\prime \prime}$ from correlations (6) and (8) (bottom). The dotted line indicates the range of 2.5 times the confidence interval of the most precise data, as discussed in the main part of this work. Error bars are omitted in the bottom plot to avoid visual clutter. For clarity, the out-of-range data points are omitted in both, the top and bottom plot. The black filled star indicates the compressibility factor at the critical point according the base correlation. 


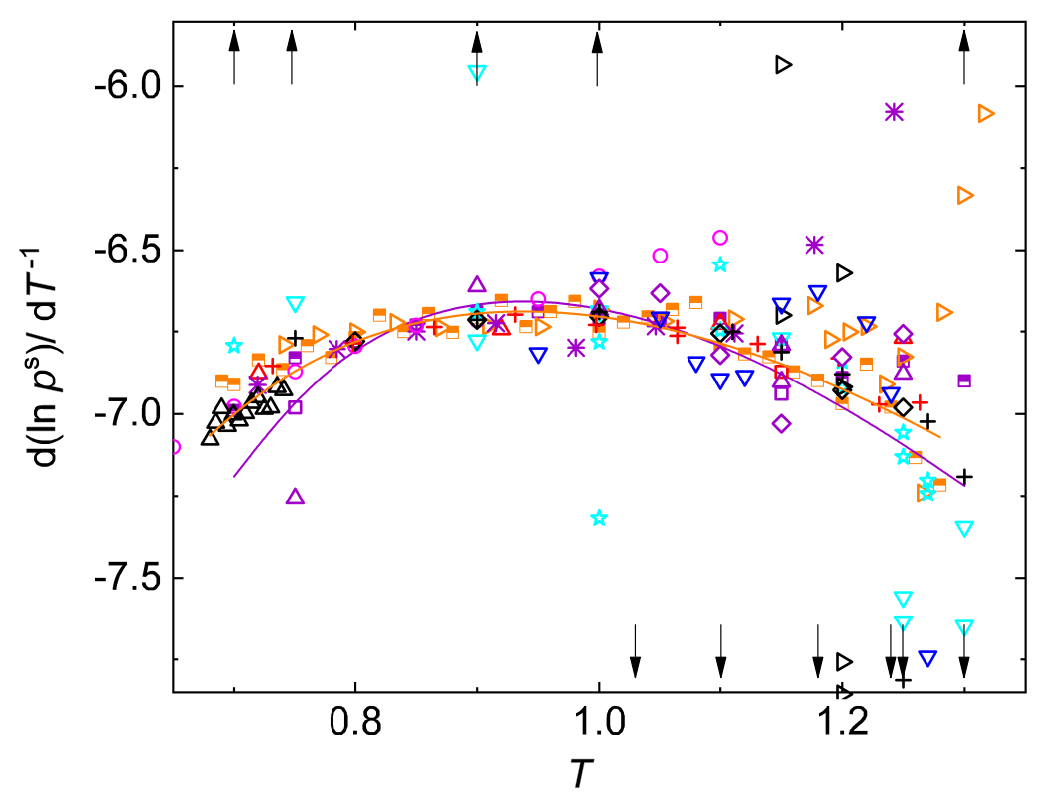

\begin{tabular}{|c|c|}
\hline & Eq. (6) \\
\hline & Eq. (22) from Ref. ${ }^{12}$ \\
\hline$\square$ & Adams $^{60}$ \\
\hline$\circ$ & Adams $^{58}$ \\
\hline$\Delta$ & Agrawal \& Kofke ${ }^{106}$ \\
\hline मे & Betancourt-Cárdenas et al. ${ }^{130}$ \\
\hline$\square$ & Hansen \& Verlet ${ }^{20}$ \\
\hline$\Delta$ & Janecek $^{131}$ \\
\hline$\diamond$ & Janecek et al. ${ }^{135,136}$ \\
\hline$\triangleleft$ & Kioupis et al. ${ }^{124}$ \\
\hline$\triangleright$ & Kofke ${ }^{104}$ \\
\hline
\end{tabular}

- Lotfi et al. ${ }^{71}$

+ Mick et al. ${ }^{23}$

$\triangle$ Panagiotopoulos $^{100}$

$\nabla$ Panagiotopoulos et al. ${ }^{101}$

$\nabla$ Plackov \& Sadus ${ }^{110}$

$\diamond$ Sadus \& Prausnitz ${ }^{108}$

$\triangleright$ Smit \& Frenkel ${ }^{103}$

+ Stoll et al. ${ }^{128}$

- this work

* Werth et al. ${ }^{137}$

Figure S3: Clausius-Clapeyron test according to Eq. (10). Symbols indicate the RHS and the lines the LHS of Eq. (10). The orange line indicates Eq. (6). The purple line is Eq. (22) from Ref. ${ }^{71}$ For clarity, the numeric values of the out-of-range data points are omitted in the plot; they lie in the range of $\mathrm{d}\left(\ln p^{s}\right) / \mathrm{d}\left(T^{-1}\right)=-31$ to -0.8 . 

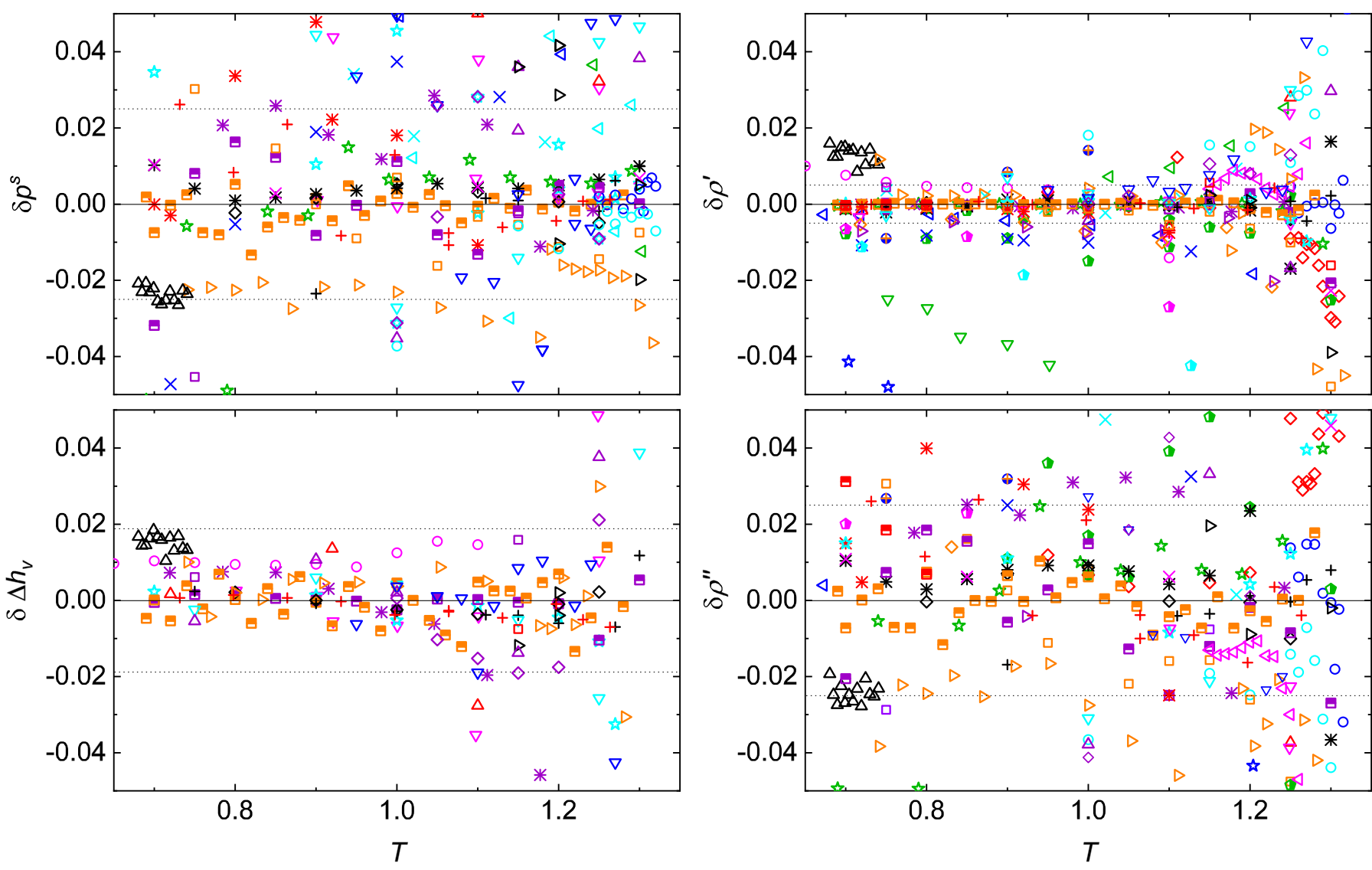

\begin{tabular}{|c|c|}
\hline 口 & Adams $^{60}$ \\
\hline 0 & Adams $^{58}$ \\
\hline$\Delta$ & Agrawal \& Kofke ${ }^{106}$ \\
\hline$\nabla$ & Anisimov et al. ${ }^{115}$ \\
\hline$\diamond$ & Baidakov et al. ${ }^{117}$ \\
\hline$\triangleleft$ & Baidakov et al. ${ }^{129}$ \\
\hline$\triangleright$ & Baidakov et al. $^{123}$ \\
\hline th & Betancourt-Cárdenas et al. ${ }^{130}$ \\
\hline$\Delta$ & Chapela et al. ${ }^{99}$ \\
\hline च & Chen et al. ${ }^{24}$ \\
\hline$x$ & Errington $^{125}$ \\
\hline * & Errington ${ }^{126,127}$ \\
\hline - & Galliero et al. ${ }^{132}$ \\
\hline+ & Guo et al. ${ }^{111}$ \\
\hline & Guo \& Lu ${ }^{112}$ \\
\hline
\end{tabular}

\begin{tabular}{|c|c|}
\hline & Hansen \& Verlet $^{20}$ \\
\hline & Holcomb et al. ${ }^{105}$ \\
\hline & Hunter \& Reinhardt ${ }^{107}$ \\
\hline & Janecek $^{131}$ \\
\hline & Janecek et al. ${ }^{135}$ \\
\hline & Janecek et al. ${ }^{135,136}$ \\
\hline & Kioupis et al. $^{124}$ \\
\hline & Kofke ${ }^{104}$ \\
\hline & Lee $^{98}$ \\
\hline & Lotfi et al. ${ }^{71}$ \\
\hline & Martin \& Siepmann ${ }^{113}$ \\
\hline & Martínez-Ruiz et al. ${ }^{134}$ \\
\hline & Mecke et al. ${ }^{109}$ \\
\hline & Mick et al. ${ }^{23}$ \\
\hline & Nijmeijer et al. ${ }^{102}$ \\
\hline
\end{tabular}

\begin{tabular}{|c|c|}
\hline 口 & Okumura \& Yonezawa ${ }^{118,119}$ \\
\hline o & Okumura \& Yonezawa ${ }^{121,122}$ \\
\hline & Panagiotopoulos ${ }^{100}$ \\
\hline & Panagiotopoulos et al. ${ }^{101}$ \\
\hline & Plackov \& Sadus ${ }^{110}$ \\
\hline & Potoff \& Panagiotopoulos ${ }^{116}$ \\
\hline & Sadus \& Prausnitz ${ }^{108}$ \\
\hline & Sadus $^{133}$ \\
\hline & Shi \& Johnson ${ }^{120}$ \\
\hline & Smit \& Frenkel ${ }^{103}$ \\
\hline & Stephan \& Hasse ${ }^{138}$ \\
\hline & Stoll et al. ${ }^{128}$ \\
\hline & this work \\
\hline & Trokhymchuka \& Alejandre ${ }^{114}$ \\
\hline & Werth et al. ${ }^{137}$ \\
\hline
\end{tabular}

Figure S4: Relative deviations of the vapor-liquid equilibrium data for the vapor pressure $p^{s}$, saturated liquid density $\rho^{\prime}$, saturated vapor density $\rho^{\prime \prime}$, and enthalpy of vaporization $\Delta h_{v}$ from correlations (6) - (9) as a function of the temperature $T$. Error bars were omitted to avoid visual clutter. The dotted lines indicates the range of 2.5 times the confidence interval of the most precise data $\delta x$, as discussed in the main part of this work. Error bars are omitted in the plots to avoid visual clutter. For clarity, the out-of-range data points are omitted in the deviation plots. 

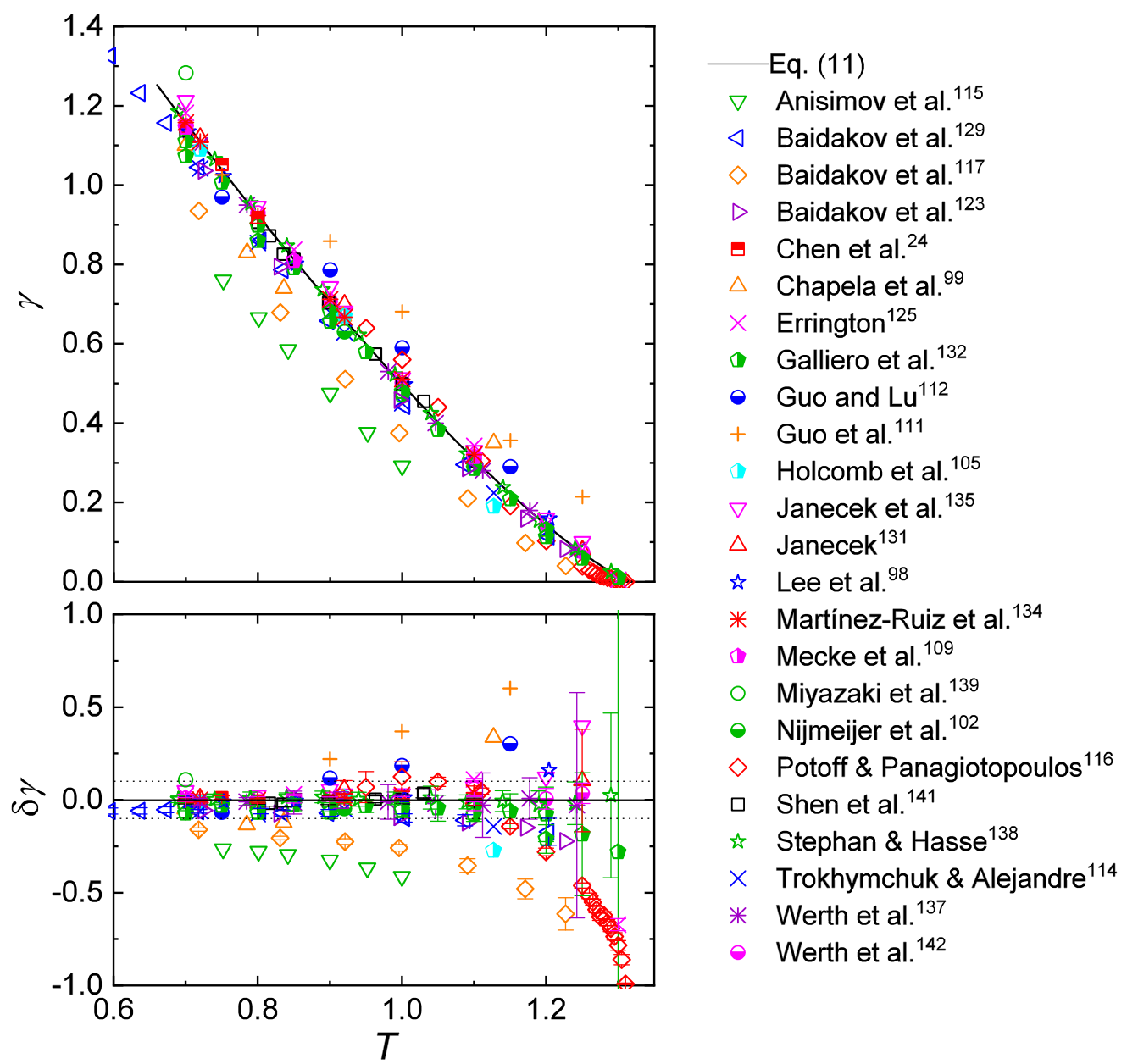

Figure S5: Surface tension of the LJ fluid as a function of the temperature (top) and the corresponding relative deviation plot (bottom). The black line indicates Eq. (11). Symbols indicate computer experiment data. For clarity, numerical values for out-of-range data points in the vicinity of the critical temperature in the deviation plot are omitted. The dotted lines indicates the range of 2.5 times the confidence interval of the most precise data as discussed in the main part of this work. 